\title{
1997 \\ ANNUAL GROUND CONTROL OPERATING PLAN FOR THE WASTE ISOLATION PILOT PLANT
}

\author{
WESTINGHOUSE \\ GEOTECHNICAL ENGINEERING
}

HH

PROCESSED FROM BEST AVALLABLE COPY

February 1997

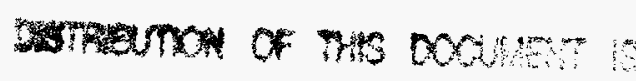




\section{DISCLAIMER}

This report was prepared as an account of work sponsored by an agency of the United States Government. Neither the United States Government nor any agency thereof, nor any of their employees, make any warranty, express or implied, or assumes any legal liability or responsibility for the accuracy, completeness, or usefulness of any information, apparatus, product, or process disclosed, or represents that its use would not infringe privately owned rights. Reference herein to any specific commercial product, process, or service by trade name, trademark, manufacturer, or otherwise does not necessarily constitute or imply its endorsement, recommendation, or favoring by the United States Government or any agency thereof. The views and opinions of authors expressed herein do not necessarily state or reflect those of the United States Government or any agency thereof. 


\section{DISCLAIMER}

Portions of this document may be illegible electronic image products. Images are produced from the best available original document. 
This document has been reproduced directly from the best possible copy.

It is available to DOE and DOE contractors at the following addresses:

Office of Scientific and Technical Information

P.O. Box 62

Oak Ridge, TN 37831

Prices available from (615) 576-8401

Available to the public from the National Technical Information Services

U. S. Department of Commerce

5285 Port Royal Road

Springfield, VA 22161

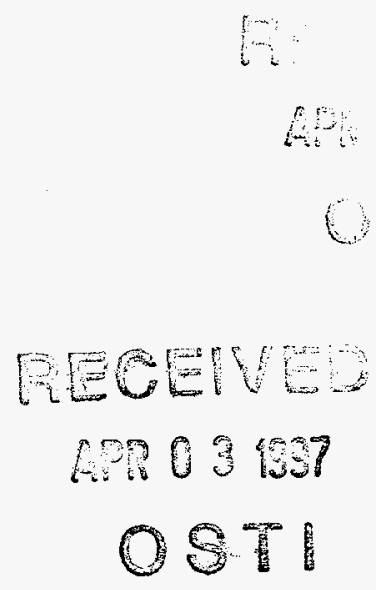

Processing and final preparation of this report was performed by the Waste Isolation Pilot Plant Management and Operating Contractor for the U.S. Department of Energy under Contract No. DE-ACO4-86AL31950. 
Processing and final preparation of this report was performed by the Waste Isolation Pilot Plant Management and Operating Contractor for the U. S. Department of Energy under Contract No. DE-AC04-86AL31950. 


\section{TABLE OF CONTENTS}

1. 0 INTRODUCTION 1

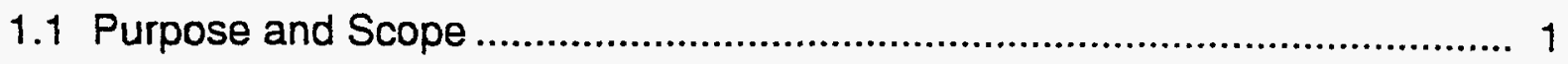

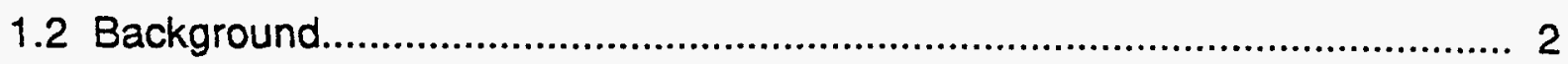

1.2.1 Summary of 1996 Ground Control Actions......................................... 2

1.2.2 Projected Ground Control Measures for 1997 .................................... 3

2. 0 CURRENT STATUS AND CONDITIONS OF UNDERGROUND OPENINGS ....... 4

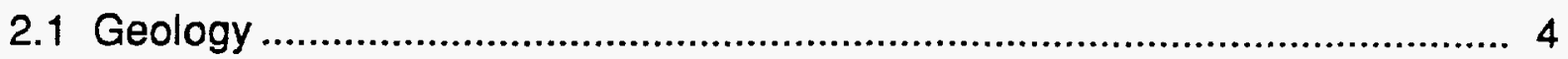

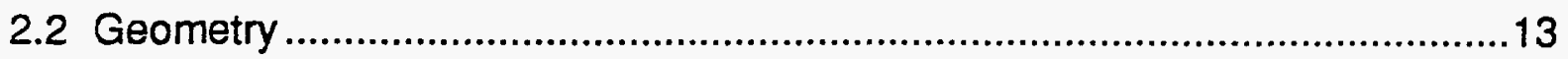

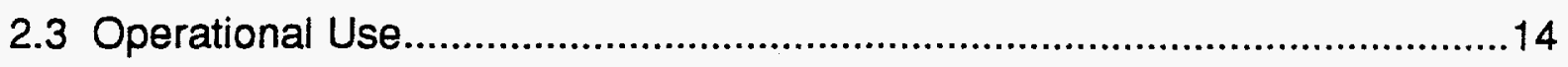

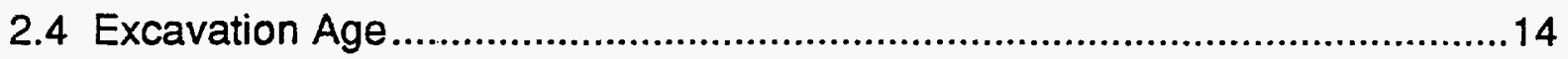

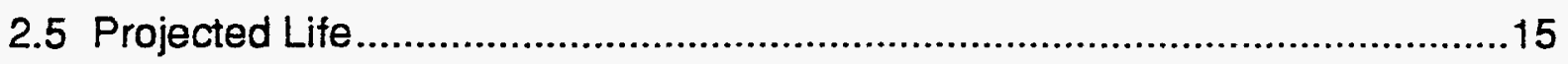

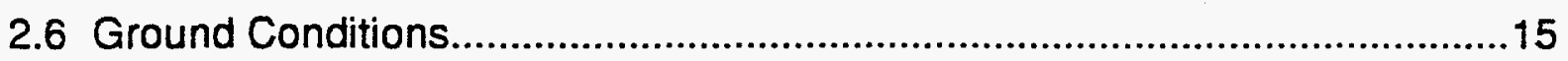

2.6.1 General Roof Beam Failure Mechanisms and Patterns......................16

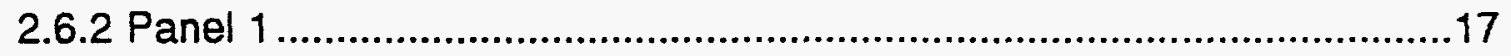

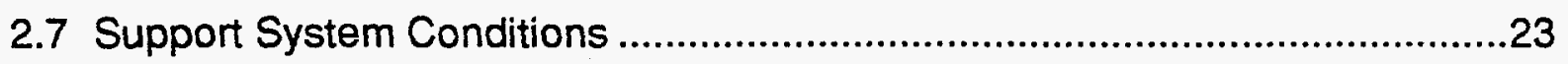

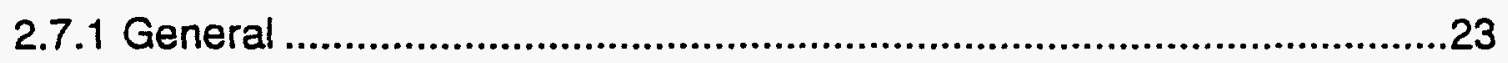

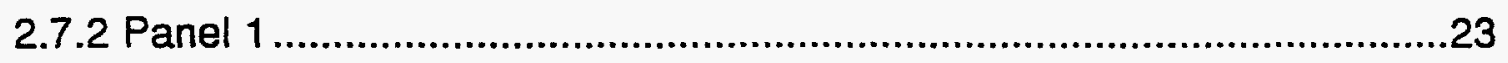

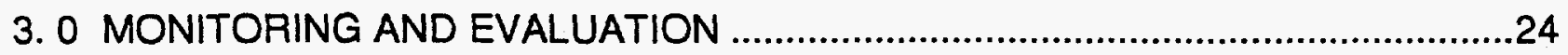

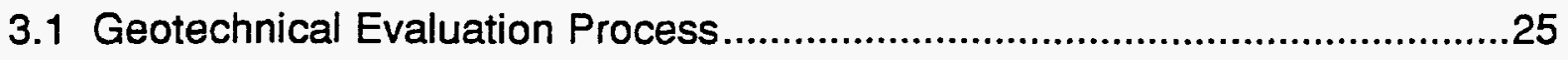

3.2 Surface Observations by Geotechnical Engineering ..................................26

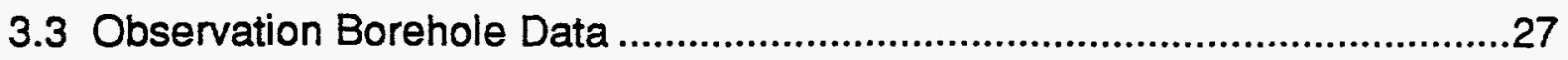

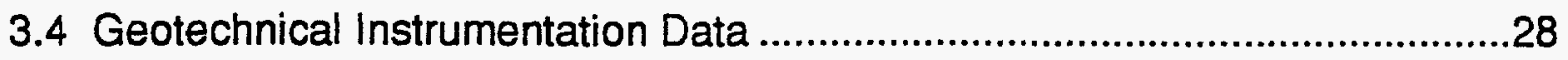

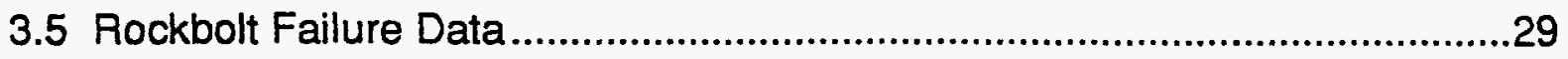

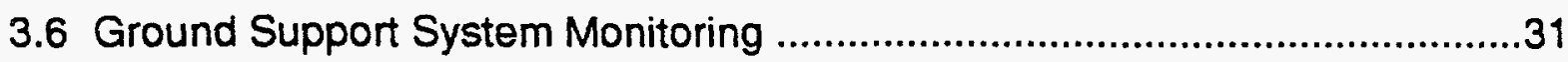

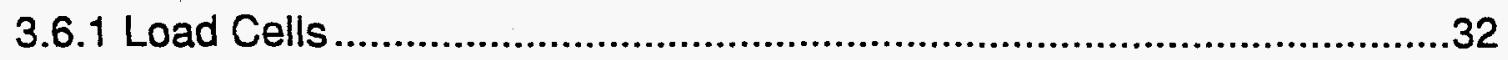

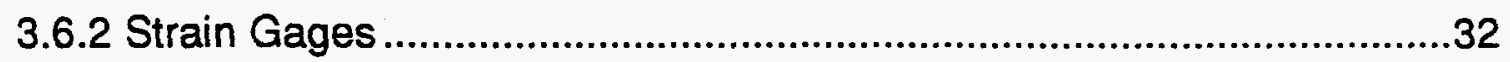

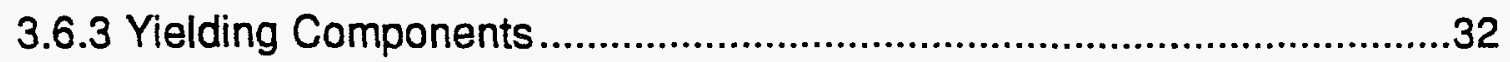

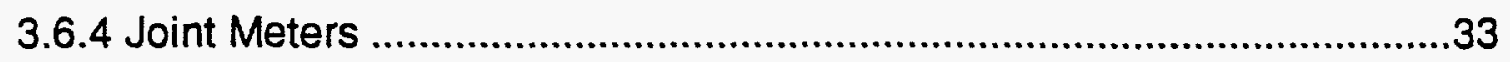

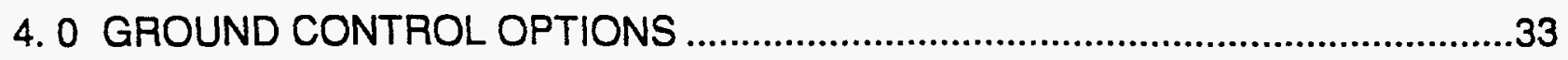

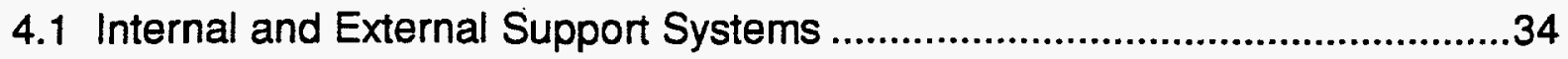




\section{TABLE OF CONTENTS}

(Continued)

4.1.1 Mechanically Anchored (Expansion Shell) Bolts..................................35

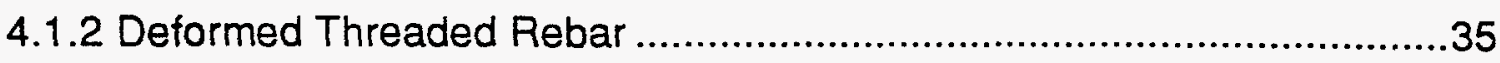

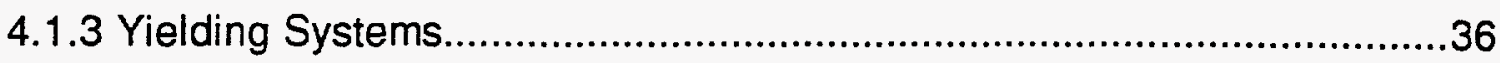

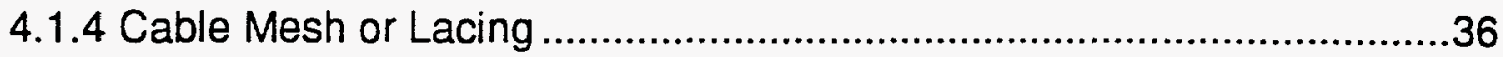

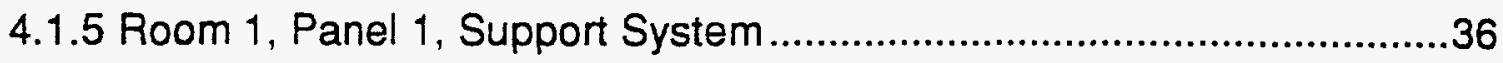

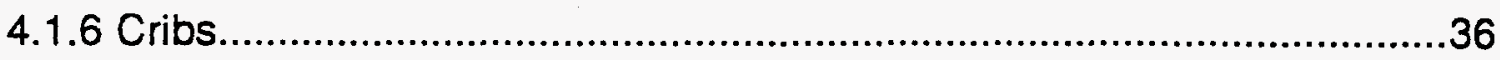

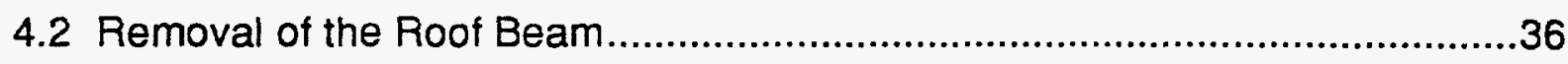

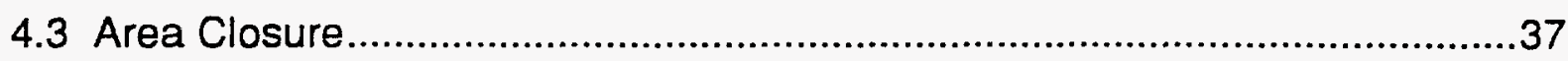

5. 0 REMEDIAL GROUND CONTROL ACTIONS IMPLEMENTED TO DATE .............37

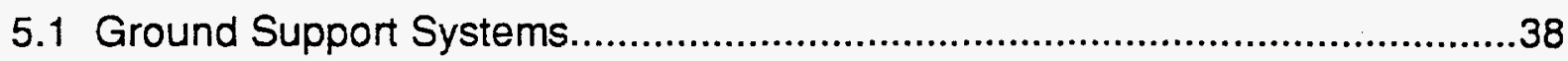

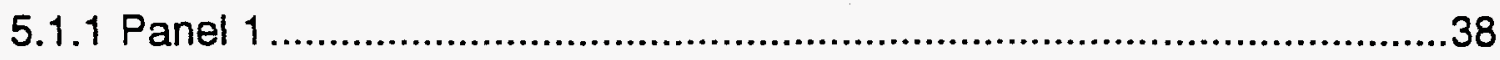

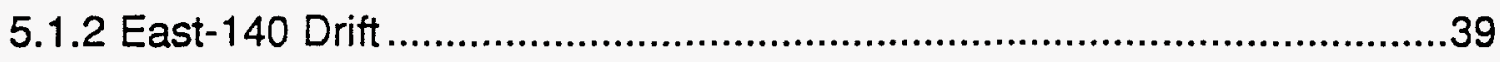

5.1.3 East-0 Drift — North-700 to North-780 ................................................40

5.1.4 East-0 and East-140 Drifts - North-780 to North-830 ........................40

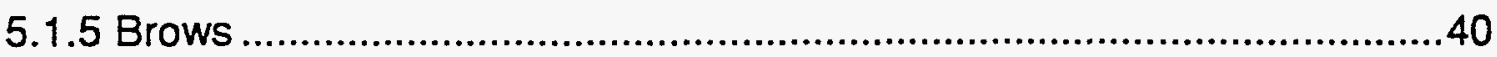

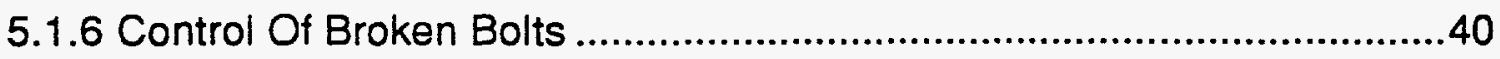

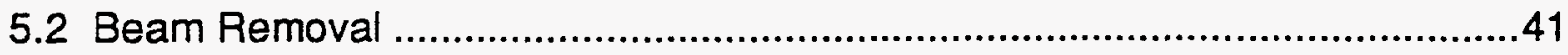

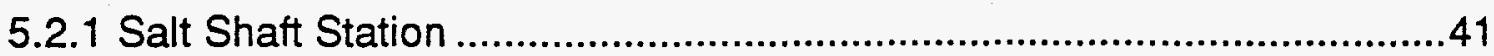

5.2.2 East-140 Drift — South (Access to Disposal Panels) ...........................42

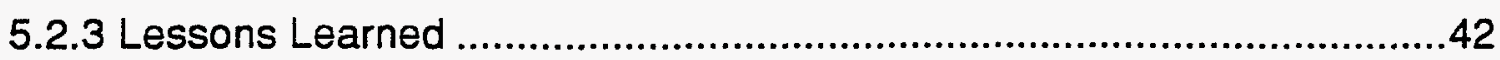

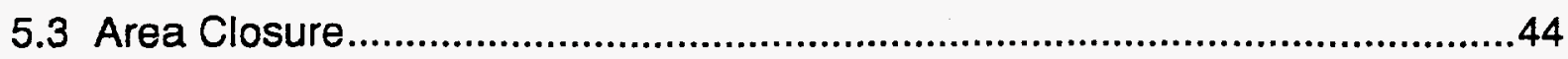

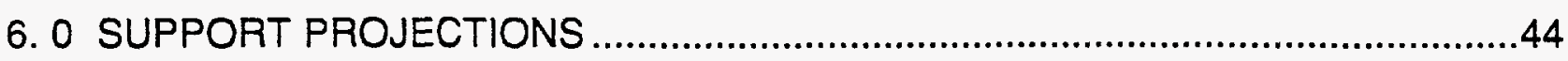

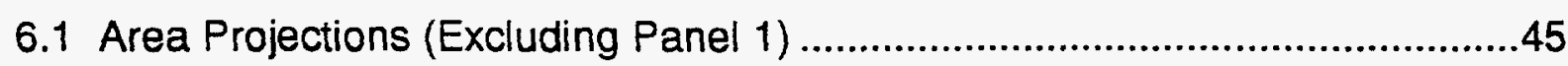

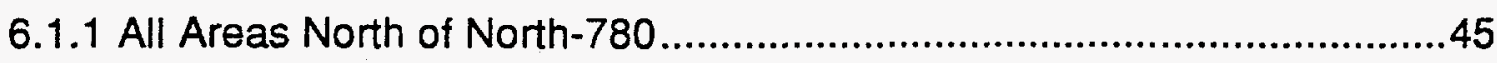

6.1.2 East-0 - North of Salt Shaft Station Brow to North-780 .....................45

6.1.3 Parts of Air Intake Shaft North Access................................................46

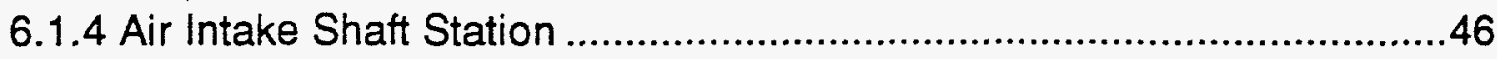

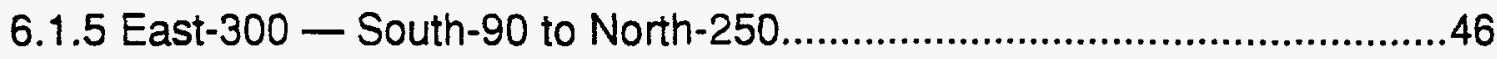

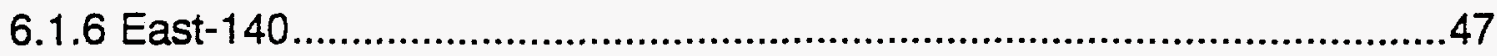

6.1.7 East-140 — South (Access to Disposal Panels) ..................................47

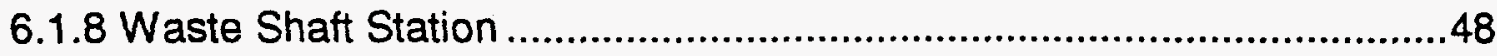




\section{TABLE OF CONTENTS}

(Continued)

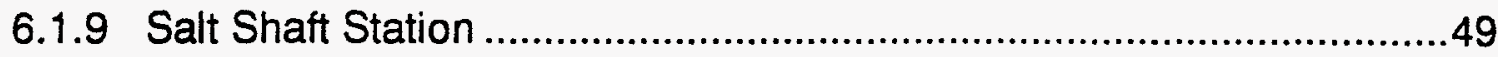

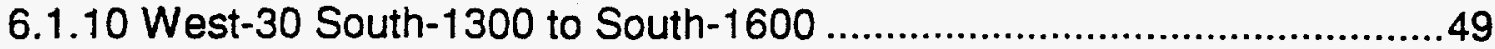

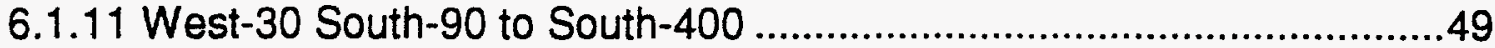

6.1.12 West-30 South-1150 Booster Fan Area...........................................49

6.1.13 South-1000 Crosscut Between West-30 and West-170 .....................50

6.1.14 South-1300 Crosscut Between West-30 and West-170 .....................50

6.1.15 South-1600 Crosscut Between West-30 and West-170 .....................50

6.2 Long-Term Low-Maintenance Areas..........................................................50

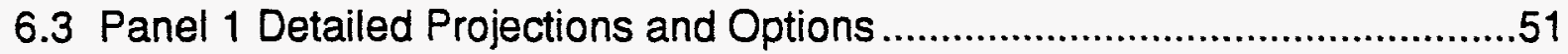

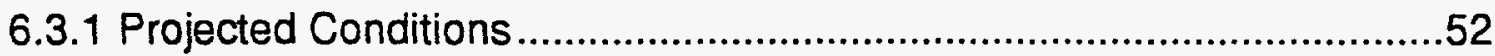

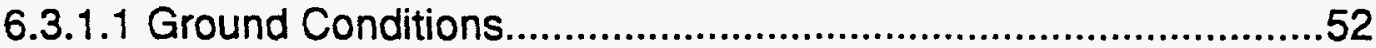

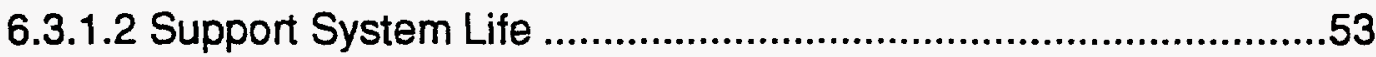

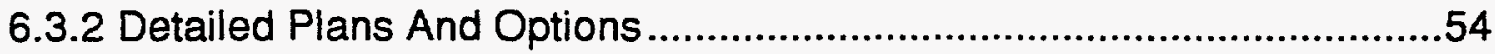

6.3.2.1 Use All of Panel 1 ..............................................................54

6.3.2.2 Receive the First Waste in Panel 1 and Use as Much of the Panel as Possible ..................................................................56

6.3.2.3 Receive the First Waste in Panel 1 and Move to Panel 2 as Soon as Possible

6.3.2.4 Close All of Panel 1 and Begin Initial Waste Emplacement in Panel 2 58

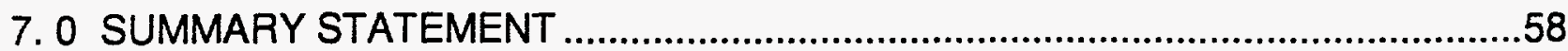

8. 0 REFERENCES

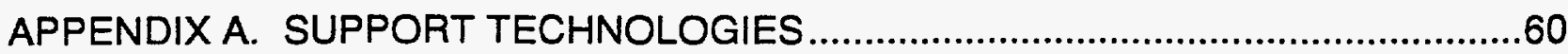

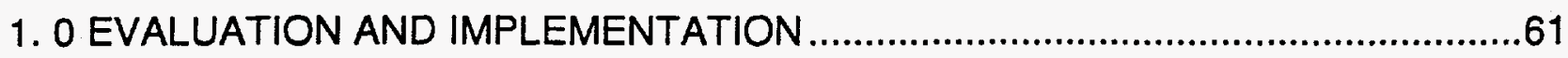

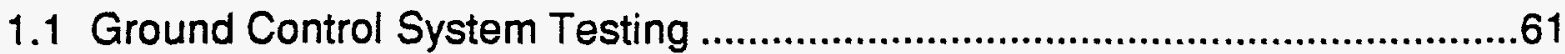

1.1.1 No. 7 Grade 60 Threaded Bar With Dywidag Slipnut ...........................62

1.1.2 No. 7 Grade 60 Threaded Bar With Yielding Rockbolt Insert ................62

1.1.3 Tensionable Cable Bolt With Dywidag Slipnut .....................................63

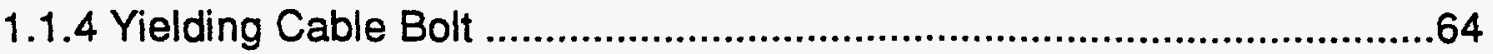

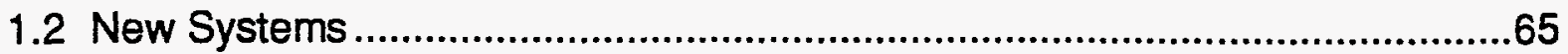




\section{TABLE OF CONTENTS}

(Continued)

2. 0 ADVANTAGES AND DISADVANTAGES OF GROUND CONTROL SYSTEMS ...65

2.1 Mechanically Anchored (Expansion Shell) Bolts .........................................65

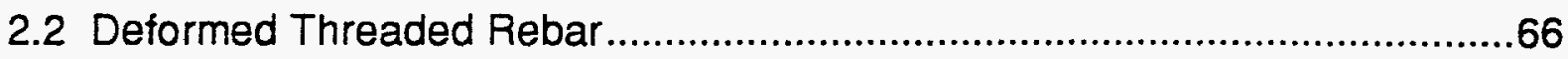

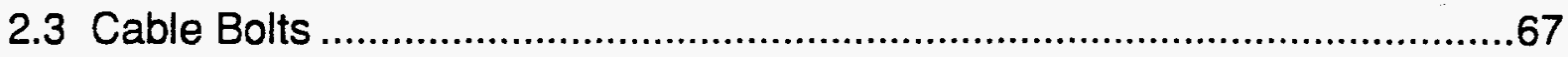

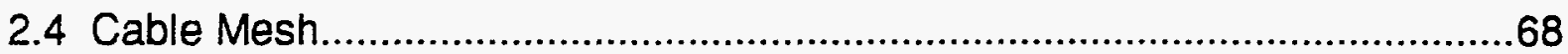

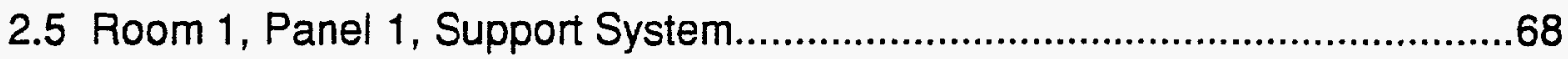

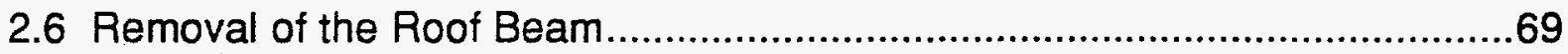

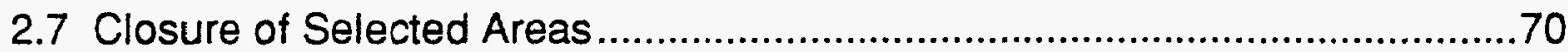




\section{LIST OF TABLES}

TABLE

PAGE

2-1 Underground Assessment Zones - Statistical Information 6

2-2 Underground Inspection Survey Summary ...................................................... 9

2-3 Panel 1 - Total Vertical and Horizontal Closure .............................................23

3-1 Ground Control System Instrumentation ........................................................31 


\section{LIST OF FIGURES}

FIGURE

PAGE

2-1 WIPP Underground Assessment Zones............................................... 5

2-2 Typical Stratigraphy at the WIPP Facility Horizon...........................................12

2-3 Generalized Fracture Development Sequence .......................................... 18

2-4 Roof Beam Offsetting and Related Bolt Loading ............................................ 19

2-5 Typical Roof Beam Fracturing ...................................................................20

2-6 Typical Roof Beam Fracturing and Related Bolt Loading ..................................21

2-7 Advanced Roof Beam Fracturing and Related Bolt Loading .............................22

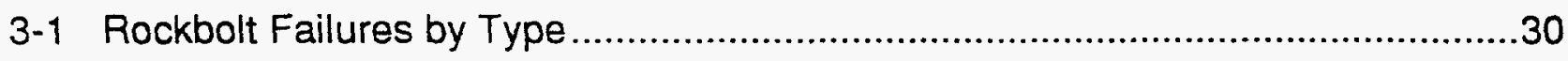

6-1 Baseline Repository Waste Emplacement Schedule ...................................51 


\subsection{INTRODUCTION}

This Annual Ground Control Operating Plan (AGCOP) is an internal guide document for short- and long-term planning for the Westinghouse Mine Operations and Engineering groups. The data collected for the plan and the evaluation of those data are most useful when used or considered immediately after collection. Use of the data becomes more difficult and less certain with time. This plan is updated annually, and each successive document supersedes the previous document. The data, evaluations, and support plans may be updated more frequently. This document is not intended to be used as a final plan for construction. Detailed plans are developed specifically for that purpose.

\subsection{Purpose and Scope}

This plan presents background information and a working guide to assist Mine Operations and Engineering in developing strategies for addressing ground control issues at the Waste Isolation Pilot Plant (WIPP). With the anticipated receipt of waste in late 1997, this document provides additional detail to Panel 1 activities and options. The plan also serves as a foundation document for development and revision of the annual Long-Term Ground Control Plan. Section 2.0 documents the current status of all underground excavations with respect to location, geology, geometry, age, ground support, operational use, projected life, and physical conditions. Section 3.0 presents the methods used to evaluate ground conditions, including visual observations of the roof, ribs, and floor, inspection of observation holes, and review of instrumentation data. Section 4.0 lists several ground support options and specific applications of each. Section 5.0 discusses remedial ground control measures that have been implemented to date. Section 6.0 presents projections and recommendations for ground control actions based on the information in Sections 2.0 through 5.0 of this plan and on a rating of the critical nature of each specific area. Section 7.0 presents a summary statement, and Section 8.0 includes references. Appendix A provides an overview and critique of ground control systems that have been, or may be, used at the site. Because of the dynamic nature of the underground openings and associated geotechnical activities, this plan will be revised as additional data are incorporated. 


\subsection{Background}

Underground excavation at WIPP began in 1982. Since that time, over 7 miles of drifts, rooms, and alcoves have been excavated with several areas, including all areas north of North-780, now closed to access. The excavations vary in geometry, geology, age, and operational use. These differences affect the selection of ground control measures; but the ability of the salt to creep or flow with time and the resulting fracture process has the greatest impact on selection of ground control systems. The result of salt creep is that strong forces, both vertical and horizontal, are exerted on any control mechanism. During the time that the underground has been active, a variety of ground control issues have been encountered ranging from minor spalling to roof falls. In general, ground control issues before 1989 were addressed on a case-by-case basis as they were identified. During 1989 and 1990, the roof in the majority of the underground openings was pattern bolted with 10-foot-long mechanical-anchor rockbolts. Since that time, the approach to ground control has evolved into a much more comprehensive and proactive program. The program consists of many aspects that include continuous visual inspections of the underground openings, an extensive geomechanical monitoring system, numerical modeling studies, tracking and analysis of rockbolt failures, implementation of selective ground control procedures, and comprehensive in situ and laboratory testing and evaluation of ground control components and systems.

Because of delays in the schedule for waste receipt, the ground control program has been adapted to address the requirement that many openings will be maintained for a longer period of time than originally anticipated. The development of plans for areas that involve waste handling/emplacement are based on the "Baseline Repository Waste Emplacement Schedule" [Garcia, 1996], which specifies receipt of waste in November of 1997. The ground control program at WIPP has produced a greater understanding of the failure mechanisms involved in the mine roof and the support systems installed in it. As the database of information grows with time, the ability to preserve and maintain optimal ground conditions and to predict and/or mitigate hazardous conditions improves.

\subsubsection{Summary of 1996 Ground Control Actions}

This plan addresses ground control issues on a contemporary basis. Major ground control measures implemented in calendar year 1996 include the following: 
- Supplemental pattern bolting with threaded-bar bolts was performed in Panel 1, Room 3, and is in progress in South-1950.

- Roof beam removal was completed in the East-140 Drift from South-1300 to South-1950. Six-foot mechanical-anchor bolts were installed following the beam removal.

- Several arrays of yielding cable bolts were installed in the East-140 Drift between North-460 and North-780.

- Supplemental pattern bolting with threaded-bar bolts was performed in East-140 between North-170 and North-460.

- The East-0 and East-140 Drifts were closed to access north of North-780. Ground control actions associated with the closure included pattern bolting with threaded bar through the North-780 intersections to approximately North-830 in both drifts and the installation of cribs just north of the barricades.

\subsubsection{Projected Ground Control Measures for 1997}

The north end of the facility was closed in 1996; and all future ground control actions will take place on the disposal horizon. With the anticipated receipt of waste in late 1997, Panel 1 conditions will be scrutinized carefully to ascertain suitability for waste disposal. Most of the panel is reinforced with supplemental threaded-bar bolt installations. Current projections call for adding additional ground control only as needed and, preferably, immediately prior to waste emplacement. With waste emplacement being a controlling parameter, ground control projections for Panel 1 include:

- Installation of supplemental support of cable lacing in Room 7.

- Installation of cable slings in Room 6 .

- Completion of pattern bolting with threaded bar in South-1950.

Detailed projections for Panel 1 are discussed in Section 6.3. Tentative projections for routine ground control actions in the remainder of the facility include the addition of supplemental pattern bolting with threaded-bar bolts. This may be required in the following areas in the next year:

- East-140 - South-700 to South-1000

- East-0 - North-150 to North-700

These projections are tentative and are based on evaluations of current conditions. 


\subsection{CURRENT STATUS AND CONDITIONS OF UNDERGROUND OPENINGS}

Conditions may necessitate ground control actions in areas not anticipated at this time. The underground has been divided into zones, based primarily on location, for the purpose of evaluating and documenting the current status of each particular zone.

Figure 2-1 presents a layout of the facility with the numeric identification of each zone. Table 2-1 lists statistical information on each zone, such as area description, roof beam dimensions, opening geometry, excavation age, ground support, and operational use. This table also gives the projected life of the zone based on its operational use. Table 2-2 lists the current physical-condition assessment of each zone based on field surveys performed in October of 1996. The information provided in Table 2-2 is based on qualitative evaluations of roof, rib, and floor fracturing and related degradation of the opening. The data presented in Tables 2-1 and 2-2 are used for making ground control projections.

\subsection{Geology}

The underground facility horizon lies within the Salado Formation. The basic constituents of the formation are near horizontal beds of clear halite (salt), argillaceous halite, and polyhalitic halite. A detailed geologic discussion of the Salado Formation can be found in Holt and Powers [1984]. Two mining horizons are located within the facility horizon: (1) the disposal horizon and (2) the experimental horizon. Within these horizons are seams of anhydrite and clay that have a significant impact on the stability of openings and the selection of ground control systems. All openings in the experimental horizon are closed and no longer accessible; therefore, any reference relative to this horizon is limited to historical perspective. Figure 2-2 shows the typical stratigraphy at the WIPP facility horizon.

From a ground control perspective, it is recognized that localized geologic conditions can have a considerable impact on the stability of an opening. Fracture development at a specific location, within a roof beam, for example, may be influenced by the clay content or other seemingly insignificant factors that may be present. 

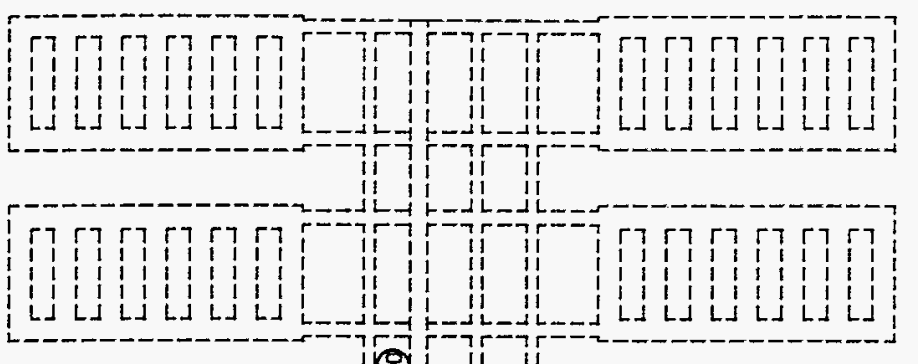

i果
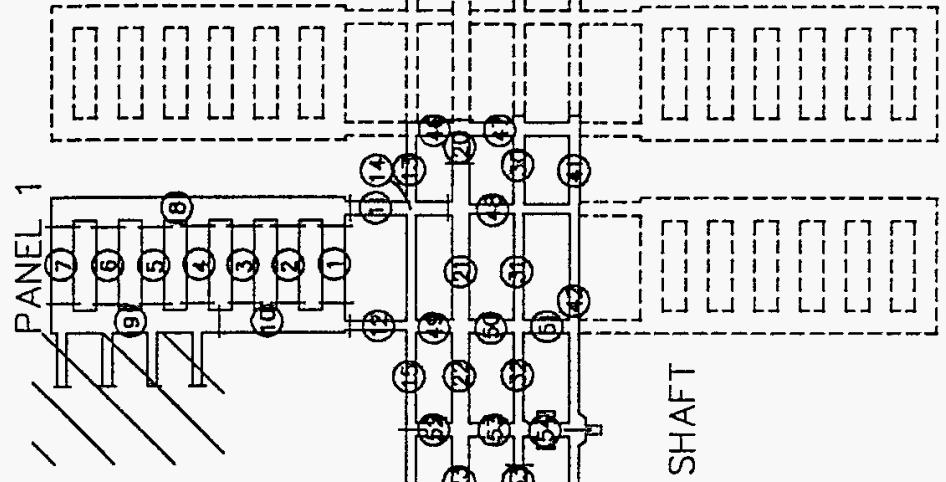

exp

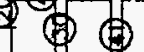

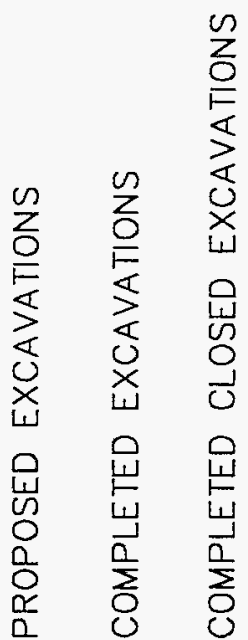
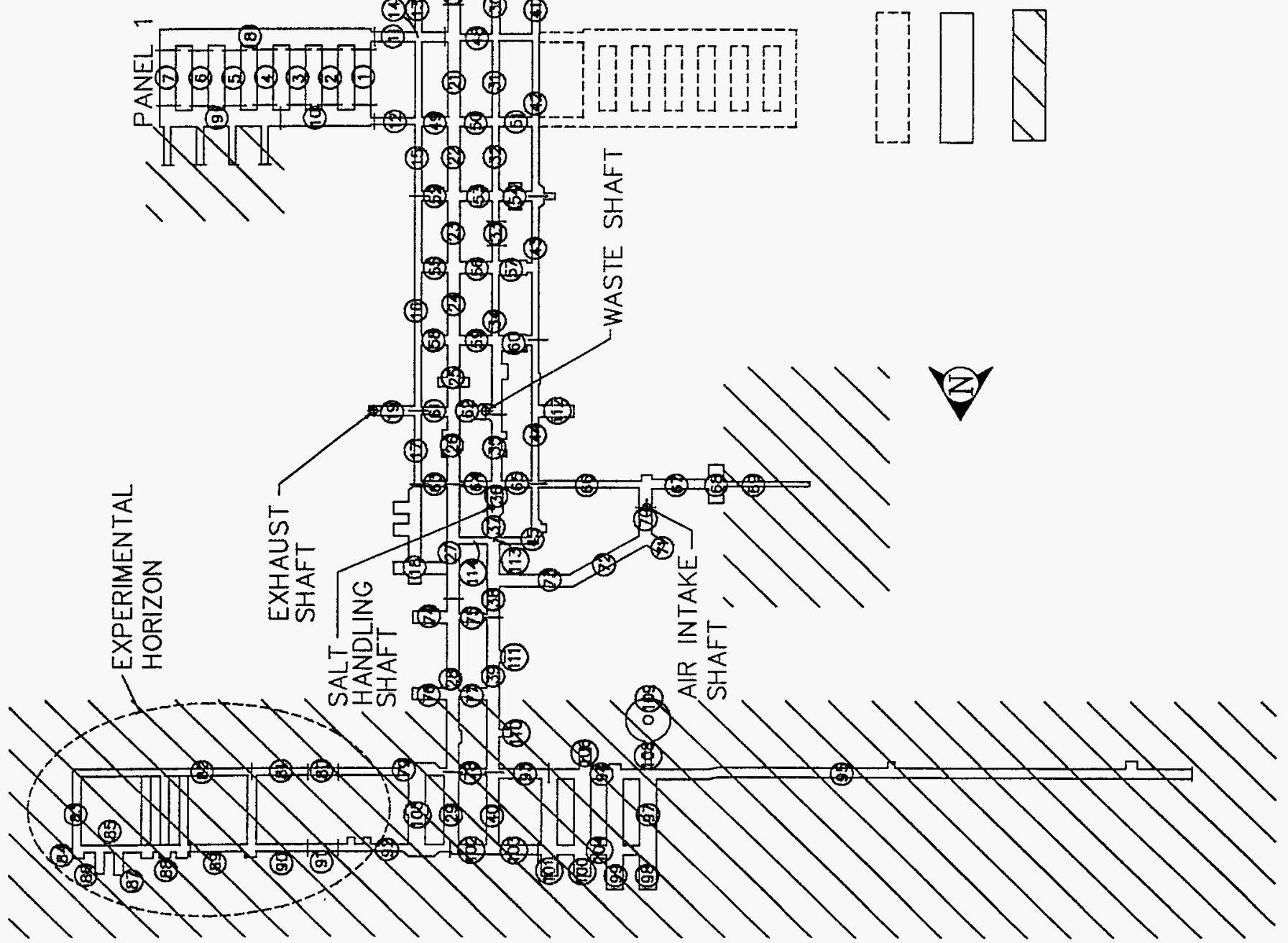

Figure 2-1. WIPP Underground Assessment Zones 
Table 2-1. Underground Assessment Zones - Statistical Information (Page 1 of 3)

\begin{tabular}{|c|c|c|c|c|c|c|c|c|c|c|}
\hline $\begin{array}{l}\text { Zone } \\
\text { Number }\end{array}$ & $\begin{array}{c}\text { Area } \\
\text { Description }\end{array}$ & $\begin{array}{c}\text { Opening } \\
\text { Dimensions } \\
\text { (ft.) }\end{array}$ & Use & $\begin{array}{l}\text { Age } \\
(y r .)\end{array}$ & $\begin{array}{l}\text { Est. } \\
\text { Life }\end{array}$ & $\begin{array}{l}\text { Bolt } \\
\text { Length } \\
(\mathrm{ft} .)\end{array}$ & $\begin{array}{c}\text { Bolt } \\
\text { Diam. } \\
\text { (in.) }\end{array}$ & $\begin{array}{l}\text { Bolt } \\
\text { Type }\end{array}$ & $\begin{array}{c}\text { Bolt } \\
\text { Spacing } \\
\text { (ft.) }\end{array}$ & $\begin{array}{c}\text { Roof Beam } \\
\text { Dimensions } \\
\text { (ft.) }\end{array}$ \\
\hline 1 & ROOM 1 PANEL 1 & $13 \times 33$ & WASTE DISP & 11 & ST & 13 & 1.000 & THRB & SPCIAL & $7 \times 33$ \\
\hline 2 & ROOM 2 PANEL 1 & $13 \times 33$ & WASTE DISP & 10 & ST & 99 & 9.999 & VARY & VARIOS & $7 \times 33$ \\
\hline 3 & ROOM 3 PANEL 1 & $13 \times 33$ & WASTE DISP & 10 & ST & 13 & 0.875 & THRB & $4.5 \mathrm{SQ}$ & $7 \times 33$ \\
\hline 4 & ROOM 4 PANEL $1(N / 2)$ & $13 \times 33$ & WASTE DISP & 9 & ST & 12 & 0.875 & THRB & $4.55 Q$ & $7 \times 33$ \\
\hline 4 & ROOM 4 PANEL 1(S/2) & $13 \times 33$ & WASTE DISP & 9 & ST & 13 & 0.875 & THRB & $4.5 \mathrm{SQ}$ & $7 \times 33$ \\
\hline 5 & ROOM 5 PANEL 1 & $13 \times 33$ & WASTE DISP & 9 & ST & 12 & 0.875 & THRB & $4.5 \mathrm{SQ}$ & $7 \times 33$ \\
\hline 6 & ROOM 6 PANEL 1 & $13 \times 33$ & WASTE DISP & 9 & ST & 12 & 0.875 & THRB & $4.5 \mathrm{SQ}$ & $7 \times 33$ \\
\hline 7 & ROOM 7 PANEL 1 & $13 \times 33$ & WASTE DISP & 9 & ST & 12 & 0.875 & THRB & $5 \times 3.8 T$ & $7 \times 33$ \\
\hline 8 & S1950 PANEL 1 & $13 \times 33$ & WASTE DISP & 10 & ST & 6 & 0.625 & $\mathrm{MECH}$ & $5 \times 5 T R I$ & $7 \times 33$ \\
\hline 9 & S1600 R4-R7 PANEL 1 & $13 \times 33$ & WASTE DISP & 9 & ST & 13 & 0.875 & THRB & $4.5 \mathrm{SQ}$ & $7 \times 33$ \\
\hline 10 & S1600 R1-R4 PANEL 1 & $13 \times 33$ & WASTE DISP & 10 & ST & 13 & 0.875 & THRB & $4.5 S Q$ & $7 \times 33$ \\
\hline 11 & S1950P1 ENT E140-300 & $13 \times 20$ & HAULAGE & 11 & ST & 6 & 0.625 & $\mathrm{MECH}$ & $5 \times 2.5 T$ & $7 \times 20$ \\
\hline 11 & S1950P1 ENT E300-520 & $13 \times 20$ & HAULAGE & 11 & ST & 6 & 0.625 & MECH & $5 \times 2.5 T$ & $7 \times 20$ \\
\hline 12 & S1600 PANEL 1 ENTRY & $12 \times 14$ & VENTILATE & 11 & ST & 6 & 0.625 & MECH & $5 \times 2.5 T$ & $8 \times 14$ \\
\hline 13 & E300 S1950-S2180 & $12 \times 14$ & VENTILATE & 11 & LT & 10 & 0.750 & MECH & $4 \times 2 \mathrm{TPI}$ & $8 \times 14$ \\
\hline 14 & E300 OVERCAST-S1950 & $15 \times 19$ & VENTILATE & 11 & LT & 0 & 0.000 & NONE & NONE & $5 \times 19$ \\
\hline 15 & $E 300 S 1300-S 1600$ & $12 \times 14$ & VENTILATE & 11 & LT & 6 & 0.625 & MECH & $5 \times 2.5 \mathrm{~T}$ & $8 \times 14$ \\
\hline 15 & E300 S1600-S1950 & $12 \times 14$ & VENTILATE & 11 & LT & 10 & 0.750 & $\mathrm{MECH}$ & $5 \times 2.5 T$ & $8 \times 14$ \\
\hline 16 & $E 300 S 400-S 1300$ & $12 \times 14$ & VENTILATE & 13 & LT & 10 & 0.750 & $\mathrm{MECH}$ & $5 \times 2.5 T$ & $8 \times 14$ \\
\hline 17 & E300 S90-S400 & $12 \times 14$ & VENTILATE & 11 & LT & 10 & 0.750 & MECH & $6 \times 3$ TRI & $8 \times 14$ \\
\hline 18 & E300 S9O-N250 & $15 \times 25$ & SHOP & 4 & 1 & 0 & 0.000 & NONE & NONE & $5 \times 25$ \\
\hline 19 & EXHST DRIFT E OF 300 & $12 \times 20$ & VENTILATE & 13 & LT & 10 & 0.750 & MECH & $5 \times 2.5 T$ & $8 \times 20$ \\
\hline 20 & E140 S OF 2180 & $8 \times 25$ & CLOSD AREA & 14 & $\mathrm{CL}$ & 0 & 0.000 & MECH & UNKNWN & $6 \times 25$ \\
\hline 20 & E140 S2050-S2180 & $15 \times 25$ & HAULAGE & 14 & LT & 8 & 0.750 & MECH & $12 \times 3$ TR & $5 \times 25$ \\
\hline 21 & E140 S1600-S1950 & $20 \times 25$ & HAULAGE & 14 & LT & 4 & 0.625 & MECH & $5 \times 5$ TRI & $6 \times 25$ \\
\hline 21 & E140 S1950-S2050 & $15 \times 25$ & HAULAGE & 14 & LT & 8 & 0.750 & MECH & $12 \times 3$ TR & $5 \times 25$ \\
\hline 22 & E140 S1300-S1600 & $20 \times 25$ & HAULAGE & 14 & LT & 5 & 0.625 & MECH & $5 \times 5$ TRI & $6 \times 25$ \\
\hline 23 & E140 S1000-S1300 & $15 \times 25$ & HAULAGE & 14 & LT & 8 & 0.750 & MECH & 12×3TR & $5 \times 25$ \\
\hline 24 & E140 $5700-51000$ & $15 \times 25$ & HAULAGE & 14 & LT & 8 & 0.750 & MECH & $12 \times 3$ TR & $5 \times 25$ \\
\hline 25 & E140 S400-S700 & $15 \times 25$ & HAULAGE & 14 & $\mathbf{L T}$ & 8 & 0.750 & MECH & 12×3TR & $5 \times 25$ \\
\hline 26 & E140 S90-S400 & $15 \times 25$ & HAULAGE & 14 & LT & 10 & 0.750 & MECH & $5 \times 2.5 \mathrm{~T}$ & $5 \times 25$ \\
\hline 27 & E140 N250-N460 & $15 \times 25$ & HAULAGE & 14 & LT & 10 & 0.750 & MECH & $5 \times 2.5 \mathrm{~T}$ & $5 \times 25$ \\
\hline 27 & E140 S90-N250 & $15 \times 25$ & HAULAGE & 14 & LT & 10 & 0.750 & MECH & $5 \times 2.5 T$ & $5 \times 25$ \\
\hline 28 & E140 N460-N780 & $15 \times 25$ & HAULAGE & 14 & LT & 99 & 9.999 & VARY & VARIOS & $5 \times 25$ \\
\hline 28 & E140 N780-N1100 & $15 \times 25$ & CLOSD AREA & 14 & $\mathrm{CL}$ & 10 & 0.750 & MECH & $5 \times 2.5 \mathrm{~T}$ & $5 \times 25$ \\
\hline 29 & E140 N1 100-N1400 & $15 \times 25$ & CLOSD AREA & 14 & $\mathrm{CL}$ & 10 & 0.750 & $\mathrm{MECH}$ & $5 \times 2.5 T$ & $5 \times 25$ \\
\hline 30 & W30 S1950-\$2180 & $12 \times 14$ & ACCESS & 8 & LT & 10 & 0.750 & $\mathrm{MECH}$ & $5 \times 2.5 \mathrm{~T}$ & $8 \times 14$ \\
\hline 31 & W30 S1600-S1950 & $12 \times 14$ & ACCESS & 11 & LT & 10 & 0.750 & MECH & $6 \times 3$ TRI & $8 \times 14$ \\
\hline 32 & W30 $\$ 1175-\$ 1300$ & $12 \times 14$ & ACCESS & 12 & LT & 10 & 0.750 & $\mathrm{MECH}$ & $5 \times 2.5 T$ & $8 \times 14$ \\
\hline 32 & W30 S1300-S1600 & $12 \times 14$ & ACCESS & 12 & LT & 6 & 0.625 & MECH & $5 \times 3 T R I$ & $8 \times 14$ \\
\hline 33 & W30 S1150 BOOST FAN & $20 \times 25$ & VENTILATE & 12 & LT & 6 & 0.750 & MECH & $4 \times 6 S Q$ & $6 \times 25$ \\
\hline 34 & W30 $\$ 400-\$ 700$ & $12 \times 20$ & ACCESS & 13 & LT & 10 & 0.750 & MECH & $5 \times 3$ TRI & $8 \times 20$ \\
\hline 34 & W30 5700-S1125 & $12 \times 14$ & ACCESS & 12 & LT & 10 & 0.750 & MECH & $5 \times 3 T R I$ & $8 \times 14$ \\
\hline 35 & W30 s90-\$400 & $12 \times 20$ & HAULAGE & 14 & $L T$ & 10 & 0.750 & MECH & $5 \times 2.5 T$ & $8 \times 20$ \\
\hline 36 & SALT SHAFT STATION & $20 \times 33$ & STATION & 15 & LT & 5 & 0.625 & MECH & $8 \times 5$ TRI & $6 \times 33$ \\
\hline 37 & EO SALT STA-N150 & $12 \times 25$ & HAULAGE & 14 & LT & 6 & 0.625 & MECH & $5 \times 3 T R I$ & $8 \times 25$ \\
\hline 38 & EO N150-N460 & $12 \times 25$ & HAULAGE & 14 & LT & 10 & 0.750 & MECH & $5 \times 2.5 \mathrm{~T}$ & $8 \times 25$ \\
\hline 39 & EO N460-N780 & $12 \times 25$ & HAULAGE & 14 & LT & 10 & 0.750 & MECH & $5 \times 2.5 \mathrm{~T}$ & $8 \times 25$ \\
\hline 39 & EO N780-N1100 & $12 \times 25$ & CLOSD AREA & 14 & $\mathrm{CL}$ & 10 & 0.750 & MECH & $5 \times 2.5 T$ & $8 \times 25$ \\
\hline 40 & EO N1100-N1400 & $12 \times 25$ & CLOSD AREA & 14 & $\mathrm{CL}$ & 10 & 0.750 & MECH & $5 \times 2.5 \mathrm{~T}$ & $8 \times 25$ \\
\hline
\end{tabular}


Table 2-1. Underground Assessment Zones - Statistical Information (Page 2 of 3)

\begin{tabular}{|c|c|c|c|c|c|c|c|c|c|c|}
\hline $\begin{array}{l}\text { Zone } \\
\text { Number }\end{array}$ & $\begin{array}{c}\text { Area } \\
\text { Description }\end{array}$ & $\begin{array}{c}\text { Opening } \\
\text { Dimensions } \\
\text { (ft.) }\end{array}$ & Use & $\begin{array}{l}\text { Age } \\
(y r .)\end{array}$ & $\begin{array}{l}\text { Est. } \\
\text { Life }\end{array}$ & $\begin{array}{l}\text { Bolt } \\
\text { Length } \\
\text { (ft.) }\end{array}$ & $\begin{array}{l}\text { Bolt } \\
\text { Diam. } \\
\text { (in.) }\end{array}$ & $\begin{array}{l}\text { Bolt } \\
\text { Type }\end{array}$ & $\begin{array}{c}\text { Bolt } \\
\text { Spacing } \\
\text { (ft.) }\end{array}$ & $\begin{array}{c}\text { Roof Beam } \\
\text { Dimensions } \\
\text { (ft.) }\end{array}$ \\
\hline 41 & W170 S1950-S2180 & $12 \times 14$ & HAULAGE & 9 & LT & 10 & 0.750 & $\mathrm{MECH}$ & $5 \times 2.5 T$ & $8 \times 14$ \\
\hline 42 & W $170 S 1300-S 1600$ & $12 \times 14$ & HAULAGE & 12 & LT & 10 & 0.750 & $\mathrm{MECH}$ & $5 \times 2.5 \mathrm{~T}$ & $8 \times 14$ \\
\hline 42 & $W 170 \$ 1600-\$ 1950$ & $12 \times 14$ & HAULAGE & 12 & $\mathrm{LT}$ & 10 & 0.750 & $\mathrm{MECH}$ & $5 \times 2.5 T$ & $8 \times 14$ \\
\hline 43 & $W 170 \$ 1000-S 1300$ & $12 \times 14$ & HAULAGE & 12 & LT & 10 & 0.750 & $\mathrm{MECH}$ & $6 \times 3$ TR! & $8 \times 14$ \\
\hline 43 & W170 S700-S1000 & $12 \times 14$ & HAULAGE & 12 & LT & 10 & 0.750 & $\mathrm{MECH}$ & $6 \times 3$ TRI & $8 \times 14$ \\
\hline 44 & W170 S90-S700 & $12 \times 14$ & HAULAGE & 13 & LT & 10 & 0.750 & $\mathrm{MECH}$ & $6 \times 3 T R I$ & $8 \times 14$ \\
\hline 45 & W17ON150 S9O/EO & $12 \times 14$ & HAULAGE & 13 & LT & 10 & 0.750 & $\mathrm{MECH}$ & 6x3TRI & $8 \times 14$ \\
\hline 46 & S2180 E140-E300 & $13 \times 20$ & HAULAGE & 11 & 1 & 10 & 0.750 & MECH & $5 \times 2.5 T$ & $7 \times 20$ \\
\hline 47 & S2180 W30-E140 & $13 \times 20$ & HAULAGE & 9 & 1 & 10 & 0.750 & $\mathrm{MECH}$ & $5 \times 2.5 \mathrm{~T}$ & $7 \times 20$ \\
\hline 47 & S2180 W30-W 170 & $13 \times 20$ & HAULAGE & 9 & 1 & 10 & 0.750 & MECH & $5 \times 2.5 T$ & $7 \times 20$ \\
\hline 48 & S1950 W30-E140 & $12 \times 14$ & CROSS & 11 & 1 & 10 & 0.750 & $\mathrm{MECH}$ & $5 \times 2.5 T$ & $8 \times 14$ \\
\hline 48 & S1950 W30-W 170 & $12 \times 14$ & CROSS & 10 & 1 & 10 & 0.750 & MECH & $5 \times 2.5 T$ & $8 \times 14$ \\
\hline 49 & S1600 E140-E300 & $12 \times 20$ & CROSS & 11 & 1 & 10 & 0.750 & MECH & $6 \times 4$ TRI & $8 \times 20$ \\
\hline 50 & $S 1600$ E140-W30 & $12 \times 20 / 27$ & CROSS & 13 & 1 & 10 & 0.750 & $\mathrm{MECH}$ & $5 \times 3$ TRI & $8 \times 20 / 27$ \\
\hline 51 & S1600 W30-W 170 & $12 \times 20$ & CROSS & 11 & 1 & 10 & 0.750 & $\mathrm{MECH}$ & $5 \times 2.5 T$ & $8 \times 20$ \\
\hline 52 & $S 1300$ E140-E300 & $12 \times 25$ & VENTILATE & 13 & 1 & 10 & 0.750 & MECH & $5 \times 3 T R I$ & $8 \times 25$ \\
\hline 53 & S1300 E140-W30 & $12 \times 20$ & OFFICES & 12 & 1 & 6 & 0.625 & MECH & 6×3TRI & $8 \times 20$ \\
\hline 54 & $S 1300$ W30-W 170 & $14 \times 20$ & SHOP & 12 & 1 & 99 & 9.999 & MECH & $4 \times 2 T R I$ & $6 \times 20$ \\
\hline 55 & S1000 E140-E300 & $12 \times 20$ & CROSS & 11 & 1 & 10 & 0.750 & $\mathrm{MECH}$ & $5 \times 5 T R I$ & $8 \times 20$ \\
\hline 56 & $\$ 1000$ E140-W30 & $12 \times 25$ & CROSS & 13 & 1 & 10 & 0.750 & MECH & $5 \times 2.5 T$ & $8 \times 25$ \\
\hline 57 & S1000 W30-W 170 & $12 \times 33$ & OFFICES & 12 & 1 & 10 & 0.750 & $\mathrm{MECH}$ & $5 \times 2.5 T$ & $8 \times 33$ \\
\hline 58 & S700 E140-E300 & $14 \times 33$ & SHOP & 13 & 1 & 10 & 0.750 & $\mathrm{MECH}$ & $5 \times 5$ TRI & $6 \times 33$ \\
\hline 59 & S700 E140-W30 & $12 \times 20$ & HAULAGE & 12 & 1 & 10 & 0.750 & $\mathrm{MECH}$ & $6 \times 3$ TRI & $8 \times 20$ \\
\hline 60 & 5700 W $30-W 170$ & $12 \times 32$ & OFFICES & 13 & 1 & 6 & 0.625 & $\mathrm{MECH}$ & $5 \times 2.5 \mathrm{~T}$ & $8 \times 32$ \\
\hline 61 & S400 E140-E300 & VARIES & VENTILATE & 13 & LT & 10 & 0.750 & MECH & $5 \times 3 T R 1$ & VARIES \\
\hline 62 & WASTE SHAFT STATION & $16 \times 22$ & STATION & 14 & LT & 10 & 0.750 & MECH & $5 \times 5 T R I$ & $4 \times 22$ \\
\hline 63 & S90 E140-E300 & $8 \times 12$ & ACCESS & 11 & LT & 10 & 0.750 & MECH & $5 \times 2.5 \mathrm{~T}$ & $12 \times 12$ \\
\hline 64 & S90 EO-E140 & $12 \times 25$ & ELECT SUBS & 11 & 1 & 0 & 0.000 & NONE & NONE & $8 \times 25$ \\
\hline 65 & 590 W30-W 170 & $12 \times 14$ & ACCESS & 12 & LT & 10 & 0.750 & MECH & $5 \times 2.5 T$ & $8 \times 14$ \\
\hline 66 & S90 W 170 -AIS & $12 \times 14$ & VENTILATE & 9 & 1 & 10 & 0.750 & $\mathrm{MECH}$ & 6x3TRI & $8 \times 14$ \\
\hline 67 & S90 AIS-Q & $12 \times 20$ & EXPERIMEN & 8 & ST & 10 & 0.750 & MECH & $6 \times 3 T R I$ & $8 \times 20$ \\
\hline 68 & Q ALCOVE & $15 \times 30$ & EXPERIMEN & 8 & ST & 6 & 0.625 & MECH & $6 \times 6 \mathrm{TRI}$ & $5 \times 30$ \\
\hline 69 & Q ROOM & 9.5RND & CLOSD & 8 & $\mathrm{CL}$ & 0 & 0.000 & MECH & NONE & N/A \\
\hline 70 & AIS STATION@ @HAFT & $20 \times 25$ & STATION & 9 & LT & 12 & 0.750 & $\mathrm{MECH}$ & $6 \times 6 \mathrm{TRI}$ & $6 \times 25$ \\
\hline 70 & AIS STATION LOW BRWS & $12 \times 25$ & STATION & 9 & LT & 6 & 0.750 & $\mathrm{MECH}$ & $6 \times 6 \mathrm{TRI}$ & $8 \times 25$ \\
\hline 71 & ROOMV & $12 \times 25$ & EXPERIMEN & 9 & ST & 4 & 0.750 & $\mathrm{MECH}$ & $5 \times 2.5 T$ & $8 \times 25$ \\
\hline 72 & AIS ACCESS N215 & $13 \times 25$ & VENTILATE & 9 & LT & 10 & 0.750 & $\mathrm{MECH}$ & $5 \times 2.5 \mathrm{~T}$ & $7 \times 25$ \\
\hline 73 & N300 OE-WEST & $13 \times 25$ & VENTILATE & 9 & LT & 10 & 0.750 & $\mathrm{MECH}$ & $5 \times 3$ TRI & $7 \times 25$ \\
\hline 74 & E140 N460 ALCOVE & $13 \times 25$ & OFFICES & 6 & 1 & 10 & 0.750 & $\mathrm{MECH}$ & $5 \times 2.5 \mathrm{~T}$ & $7 \times 25$ \\
\hline 75 & N460 EO-E140 & $13 \times 25$ & ACCESS & 14 & 1 & 10 & 0.750 & $\mathrm{MECH}$ & $6 \times 3 T R I$ & $7 \times 25$ \\
\hline 76 & E140 N780 ALCOVE & $13 \times 25$ & STORAGE & 6 & 1 & 10 & 0.750 & $\mathrm{MECH}$ & $5 \times 2.5 T$ & $7 \times 25$ \\
\hline 77 & N780 EO-E140 SHOP & $13 \times 25$ & SHOP & 14 & 1 & 10 & 0.750 & MECH & $5 \times 2.5 \mathrm{~T}$ & $7 \times 25$ \\
\hline 78 & N1100 EO-E140 & $12 \times 14$ & CLOSD & 14 & $\mathrm{CL}$ & 10 & 0.750 & $\mathrm{MECH}$ & $4 \times 5$ TRI & $8 \times 14$ \\
\hline 79 & N1100 E140-E300 & $12 \times 24$ & CLOSD & 13 & $\mathrm{CL}$ & 6 & 0.750 & MECH & $5 \times 2.5 \mathrm{~T}$ & $8 \times 24$ \\
\hline 79 & N1 100 E300-RAMP & $9 \times 14$ & CLOSD & 13 & $\mathrm{CL}$ & 10 & 0.625 & MECH & $5 \times 5 T R I$ & $8 \times 14$ \\
\hline 80 & N1100 RAMP & $9 \times 14$ & CLOSD & 13 & $C L$ & 6 & 0.750 & MECH & $5 \times 2.5 \mathrm{~T}$ & VARIES \\
\hline 81 & N1100 RAMP-ROOM B & $9 \times 14$ & CLOSD & 13 & $\mathrm{CL}$ & 6 & 0.750 & MECH & $5 \times 2.5 \mathrm{~T}$ & $8 \times 14$ \\
\hline 82 & N1100 ROOMS B-D & $9 \times 14$ & CLOSD & 13 & $\mathrm{CL}$ & 6 & 0.750 & $\mathrm{MECH}$ & $5 \times 2.5 \mathrm{~T}$ & $8 \times 14$ \\
\hline 83 & ROOM D & $18 \times 18$ & CLOSD & 13 & $\mathrm{CL}$ & 10 & 0.875 & THRB & $5 \times 5 T R I$ & $5 \times 14$ \\
\hline
\end{tabular}


Table 2-1. Underground Assessment Zones - Statistical Information (Page 3 of 3)

\begin{tabular}{|c|c|c|c|c|c|c|c|c|c|c|}
\hline $\begin{array}{l}\text { Zone } \\
\text { Number }\end{array}$ & $\begin{array}{c}\text { Area } \\
\text { Description }\end{array}$ & $\begin{array}{c}\text { Opening } \\
\text { Dimensions } \\
\text { (ft.) }\end{array}$ & Use & $\begin{array}{l}\text { Age } \\
(y r .)\end{array}$ & $\begin{array}{l}\text { Est. } \\
\text { Life }\end{array}$ & $\begin{array}{c}\text { Bolt } \\
\text { Length } \\
\text { (ft.) }\end{array}$ & $\begin{array}{c}\text { Bolt } \\
\text { Diam. } \\
\text { (in.) }\end{array}$ & $\begin{array}{l}\text { Bolt } \\
\text { Type }\end{array}$ & $\begin{array}{l}\text { Patt. Bolt } \\
\text { Spacing } \\
\text { (ft.) }\end{array}$ & $\begin{array}{c}\text { Roof Beam } \\
\text { Dimensions } \\
\text { (ft.) }\end{array}$ \\
\hline 84 & ROOMM & $11 \times 24$ & CLOSD & 13 & $\mathrm{CL}$ & 4 & 0.625 & MECH & $5 \times 5 S Q$ & $6 \times 24$ \\
\hline 85 & N1400 ROOMS A3-D & $12 \times 14$ & CLOSD & 13 & $\mathrm{CL}$ & 6 & 0.750 & $\mathrm{MECH}$ & $4 \times 3 T R I$ & $5 \times 14$ \\
\hline 86 & ROOM C-1 & $18 \times 18$ & CLOSD & 13 & $\mathrm{CL}$ & 10 & 0.750 & $\mathrm{MECH}$ & $5 \times 2.5 T$ & $5 \times 14$ \\
\hline 87 & ROOM C-2 & $18 \times 18$ & CLOSD & 13 & $\mathrm{CL}$ & 10 & 0.750 & $\mathrm{MECH}$ & $5 \times 3$ TRI & $5 \times 14$ \\
\hline 88 & N1400 ROOMS A1-A3 & $12 \times 14$ & CLOSD & 13 & $\mathrm{CL}$ & 6 & 0.625 & $\mathrm{MECH}$ & $4 \times 3$ TRI & $5 \times 14$ \\
\hline 89 & N1400 ROOMS A1-B & $12 \times 14$ & CLOSD & 13 & $\mathrm{CL}$ & 6 & 0.625 & $\mathrm{MECH}$ & $5 \times 3 T R I$ & $5 \times 14$ \\
\hline 90 & N1400 RAMP-ROOM B & $12 \times 14$ & CLOSD & 13 & $C L$ & 6 & 0.625 & $\mathrm{MECH}$ & 4X3TRI & $5 \times 14$ \\
\hline 91 & N1400 RAMP & $12 \times 14$ & CLOSD & 13 & $\mathrm{CL}$ & 10 & 0.750 & $\mathrm{MECH}$ & $5 \times 2.5 T$ & VARIES \\
\hline 92 & N1400 E140-RAMP & $12 \times 14$ & CLOSD & 13 & $\mathrm{CL}$ & 10 & 0.625 & $\mathrm{MECH}$ & $5 \times 2.5 T$ & $8 \times 14$ \\
\hline 93 & N1100 EO-SPDV RM1 & $12 \times 20$ & CLOSD & 14 & $\mathrm{CL}$ & 10 & 0.750 & $\mathrm{MECH}$ & $5 \times 5$ TRI & $8 \times 20$ \\
\hline 94 & N1100 SPDV ROOMS $1-4$ & $12 \times 20$ & CLOSD & 14 & $\mathrm{CL}$ & 10 & 0.750 & MECH & $5 \times 5$ TRI & $8 \times 20$ \\
\hline 95 & ROOM G AND G ACCESS & $10 \times 20$ & CLOSD & 12 & $\mathrm{CL}$ & 10 & 0.750 & $\mathrm{MECH}$ & VARIOS & $10 \times 20$ \\
\hline 97 & SPDV ROOM 4 & $13 \times 33$ & CLOSD & 14 & $\mathrm{CL}$ & 10 & 0.750 & $\mathrm{MECH}$ & $5 \times 5$ TRI & $7 \times 33$ \\
\hline 98 & ROOM L-4 & $13 \times 33$ & CLOSD & 8 & $C L$ & 10 & 0.750 & $\mathrm{MECH}$ & $5 \times 2.5 \mathrm{~T}$ & $7 \times 33$ \\
\hline 99 & ROOM L-3 & $13 \times 33$ & CLOSD & 8 & $\mathrm{CL}$ & 10 & 0.750 & $\mathrm{MECH}$ & $5 \times 2.5 T$ & $7 \times 33$ \\
\hline 100 & ROOM L-2 & $13 \times 33$ & CLOSD & 13 & $\mathrm{CL}$ & 10 & 0.750 & MECH & $4 \times 2$ TRI & $7 \times 33$ \\
\hline 101 & ROOM L-1 & $13 \times 33$ & CLOSD & 13 & $\mathrm{CL}$ & 10 & 0.750 & $\mathrm{MECH}$ & $5 \times 2.5 \mathrm{~T}$ & $7 \times 33$ \\
\hline 102 & N1420 EO-E140 & $12 \times 20$ & CLOSD & 14 & $\mathrm{CL}$ & 10 & 0.750 & $\mathrm{MECH}$ & $5 \times 2.5 T$ & $8 \times 20$ \\
\hline 103 & N1400 EO-ROOM L1 & $12 \times 20$ & CLOSD & 14 & $\mathrm{CL}$ & 10 & 0.750 & $\mathrm{MECH}$ & $5 \times 2.5 T$ & $8 \times 20$ \\
\hline 104 & N1400 ROOMS L1-L4 & $12 \times 20$ & CLOSD & 14 & $\mathrm{CL}$ & 10 & 0.750 & $\mathrm{MECH}$ & $4 \times 4 T R I$ & $8 \times 20$ \\
\hline 105 & E300 N1100-1400 SHOP & $13 \times 33$ & CLOSD & 7 & $\mathrm{CL}$ & 10 & 0.750 & $\mathrm{MECH}$ & $5 \times 2.5 T$ & $7 \times 33$ \\
\hline 106 & ROOM J & $12 \times 23$ & CLOSD & 13 & $\mathrm{CL}$ & 10 & 0.750 & MECH & $4 \times 4$ TRI & $8 \times 23$ \\
\hline 108 & ROOMHACCESS & $10 x+1$ & CLOSO & 13 & $\mathrm{CL}$ & 99 & 0.750 & $\mathrm{MECH}$ & $5 \times 5 T R I$ & $10 \times 11$ \\
\hline 109 & ROOMH & $10 \times 36$ & CLOSD & 12 & $\mathrm{CL}$ & 99 & 9.999 & $\mathrm{MECH}$ & $5 \times 5$ TRI & $10 \times 36$ \\
\hline 110 & N940 ALCOVE OE & $12 \times 14$ & CLOSD & 8 & $\mathrm{Cl}$ & 10 & 0.750 & MECH & 6x3TRI & $8 \times 14$ \\
\hline 111 & EO N620 ALCOVE & $12 \times 25$ & OFFICES & 8 & 1 & 10 & 0.625 & $\mathrm{MECH}$ & $5 \times 2.5 T$ & $8 \times 25$ \\
\hline 112 & W170 CORE STORAGE & $13 \times 25$ & STORAGE & 8 & ST & 10 & 0.750 & MECH & $5 \times 2.5 \mathrm{~T}$ & $7 \times 25$ \\
\hline 113 & EO N150 OVERCAST & $20 \times 15$ & VENITLATE & 10 & LT & 10 & 0.750 & $\mathrm{MECH}$ & $4 \times 450$ & $7 \times 15$ \\
\hline 114 & N150 OVERCAST-E140 & $10 \times 14$ & VENITLATE & 14 & LT & 10 & 0.750 & $\mathrm{MECH}$ & $8 \times 4.5 \mathrm{~T}$ & $10 \times 14$ \\
\hline
\end{tabular}

Note: For evaluation purposes, Zone 96 was combined with Zone 95, and Zone 107 was combined with Zone 94.

Age is calculated using the date of this printing and the date of completion of the first excavation sequence. The date of printing is $2 / 14 / 97$.

Zeros (e.g., 0.000) in a numerical column indicate that no information is available. Nines (e.g., 9.999) in a numerical column indicate multiple types or dimensions.

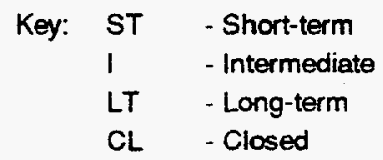

(Refer to text for detailed definitions)

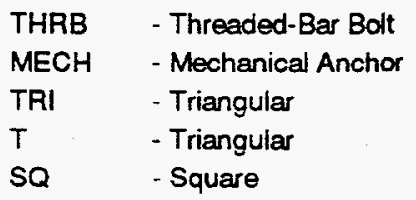


Table 2-2. Underground Inspection Survey Summary (Page 1 of 3)

\begin{tabular}{|c|c|c|c|c|c|c|c|c|c|c|c|}
\hline $\begin{array}{c}\text { Zone } \\
\text { Number }\end{array}$ & $\begin{array}{c}\text { Area } \\
\text { Description }\end{array}$ & Ass & $\begin{array}{l}\text { loof } \\
\text { ssment }\end{array}$ & $\begin{array}{l}\text { Vert. } \\
\text { Fract. }\end{array}$ & Ass & $\begin{array}{l}\text { Ribs } \\
\text { ssment }\end{array}$ & Ass & $\begin{array}{l}\infty \\
\text { smert }\end{array}$ & $\begin{array}{c}\text { Increased } \\
\text { Closure } \\
\text { Rate }\end{array}$ & $\begin{array}{l}\text { EEP/OBS } \\
\text { Assessment }\end{array}$ & $\begin{array}{l}\text { Long- } \\
\text { Projex }\end{array}$ \\
\hline 1 & ROOM 1 PANEL 1 & E2 & W2 & 1 & E1 & W1 & E2 & W2 & $Y$ & 1 & \\
\hline 2 & ROOM 2 PANEL 1 & E2 & W2 & 1 & E1 & W1 & E1 & W1 & $\mathbf{N}$ & 1 & \\
\hline 3 & ROOM 3 PANEL 1 & E3 & W2 & 2 & E1 & $W_{1}$ & E2 & $w_{1}$ & $\mathbf{Y}$ & 2 & \\
\hline 4 & ROOM 4 PANEL 1 (N/2) & E4 & W3 & 4 & E1 & $w_{1}$ & E1 & W2 & $N$ & 1 & \\
\hline 4 & ROOM 4 PANEL 1 (S/2) & E4 & w3 & 4 & E1 & W1 & $\mathrm{E} 1$ & W2 & $\mathbf{N}$ & 1 & 2 \\
\hline 5 & ROOM 5 PANEL 1 & E4 & W2 & 3 & $\mathrm{E1}$ & W1 & E2 & W2 & $N$ & 1 & 2 \\
\hline 6 & ROOM 6 PANEL 1 & E4 & W2 & 3 & E1 & $W_{1}$ & E2 & W2 & $Y$ & 1 & 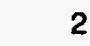 \\
\hline 7 & ROOM 7 PANEL 1 & E4 & W2 & 3 & E1 & W1 & E1 & W2 & $Y$ & 1 & 3 \\
\hline 8 & S1950 PANEL 1 & N3 & S1 & 1 & N1 & S1 & N2 & S1 & $Y$ & 1 & 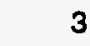 \\
\hline 9 & S1600 R4-R7 PANEL 1 & $\mathrm{~N} 2$ & S4 & 3 & N1 & S1 & N1 & S2 & $\mathbf{N}$ & 1 & \\
\hline 10 & S1600 R1-R4 PANEL 1 & $\mathrm{~N} 2$ & S3 & 1 & N1 & S1 & N2 & $\$ 2$ & $\mathbf{N}$ & 1 & \\
\hline 11 & S1950P1 ENT E140-300 & N1. & S1 & 1 & N1 & S1 & N1 & S1 & $\mathbf{N}$ & 0 & 1 \\
\hline 11 & S1950P1 ENT E300-520 & N2 & S1 & 1 & N1 & S1 & N1 & S1 & $Y$ & 0 & \\
\hline 12 & S1600 PANEL 1 ENTAY & N1 & S1 & 1 & N1 & S1 & NI & S1 & Y & 0 & \\
\hline 13 & E300 S1950-S2180 & E1 & W1 & 1 & E1 & W1 & E1 & W1 & $N$ & 0 & \\
\hline 14 & E300 OVERCAST-S1950 & E1 & W1 & 1 & NA & NA & NA & NA & $N$ & 0 & 1 \\
\hline 15 & E300 S1300-S1600 & E1 & $W_{1}$ & 1 & E1 & W1 & E1 & W1 & $N$ & 0 & \\
\hline 15 & E300 S1600-S1950 & E1 & W1 & 1 & E1 & W1 & E1 & W1 & $\mathbf{N}$ & 0 & \\
\hline 16 & E300 S400-S1300 & E1 & W1 & 1 & E1 & W1 & E2 & W2 & $Y$ & 1 & \\
\hline 17 & E300 S90-S400 & E1 & W2 & 1 & E2 & $w_{2}$ & E1 & W1 & $\mathbf{N}$ & 0 & \\
\hline 18 & E300 S90-N250 & E1 & W1 & 1 & E1 & $W_{1}$ & E1 & W1 & $\mathbf{N}$ & 1 & \\
\hline 19 & EXHST DRIFT E OF 300 & N1 & s1 & 1 & N2 & S2 & N1 & s1 & $\mathbf{N}$ & 0 & \\
\hline 20 & E140 S2050-\$2180 & $E_{1}$ & W1 & 1 & E1 & W2 & E2 & W2 & $N$ & 1 & \\
\hline 21 & E140 S1600-S1950 & E1 & W1 & 1 & E1 & WI & E1 & W1 & $Y$ & 2 & \\
\hline 21 & E140 S1950-S2050 & E1 & W2 & 1 & E1 & W1 & E3 & W1 & $Y$ & 0 & \\
\hline 22 & E140 $\$ 1300-\$ 1600$ & E1 & W1 & 1 & E1 & W1 & E2 & W2 & $Y$ & 1 & \\
\hline 23 & E140 $\$ 1000-S 1300$ & E3 & W4 & 1 & E1 & W1 & E2 & W1 & $Y$ & 3 & \\
\hline 24 & E140 $\$ 700-\$ 1000$ & E1 & W1 & 1 & E1 & W1 & E1 & W1 & $Y$ & 1 & \\
\hline 25 & E140 S400-S700 & E1 & W1 & 1 & E1 & W1 & E1 & W2 & $Y$ & 1 & \\
\hline 26 & E140 S90-S400 & E3 & W3 & 1 & E1 & W1 & E2 & W1 & $\mathbf{N}$ & 1 & 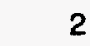 \\
\hline 27 & E140 N250-N460 & E1 & W5 & 1 & E1 & W1 & E1 & $W_{1}$ & $\mathbf{N}$ & 1 & \\
\hline 27 & E140 S90-N250 & E2 & W2 & 1 & E1 & W1 & E1 & $w_{1}$ & $\mathbf{N}$ & 1 & 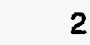 \\
\hline 28 & E140 N460-N780 & & W4 & 1 & E1 & Wi & E1 & W1 & $\mathbf{N}$ & 0 & \\
\hline 30 & W30 S1950-S2180 & E2 & W2 & 1 & E1 & $w_{1}$ & E1 & W1 & $\mathbf{N}$ & 0 & \\
\hline 31 & W30 S1600-S1950 & E1 & $W_{1}$ & 1 & E2 & W2 & E1 & W1 & $\mathbf{N}$ & 0 & \\
\hline 32 & W30 S1175-S1300 & E1 & W1 & 1 & E2 & W2 & E1 & W1 & $\mathbf{N}$ & 0 & \\
\hline 32 & W30 S1300-S1600 & E2 & W1 & 1 & $\mathrm{E} 1$ & W1 & E1 & W1 & $Y$ & 0 & \\
\hline 33 & W30 S1150 BOOST FAN & E1 & W1 & 1 & $\mathrm{E} 1$ & W1 & E1 & W1 & $N$ & 0 & \\
\hline 34 & W30 $5400-5700$ & E2 & W1 & 1 & E1 & W1 & E1 & W1 & $Y$ & 0 & \\
\hline 34 & W30 S700-S1125 & E1 & W1 & 1 & E1 & W1 & E1 & W1 & $\mathbf{N}$ & 0 & \\
\hline 35 & W30 S90-\$400 & E3 & W2 & 2 & E1 & W1 & E1 & W1 & $Y$ & 0 & 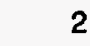 \\
\hline 36 & SALT SHAFT STATION & E1 & W1 & 2 & E2 & W2 & E1 & W1 & $Y$ & 2 & \\
\hline 37 & EO SALT STA-N150 & E3 & W3 & 1 & E1 & W1 & E3 & W1 & $N$ & 3 & \\
\hline 38 & EO N150-N460 & E3 & W3 & 3 & E1 & W1 & E2 & W2 & $Y$ & 0 & \\
\hline 39 & EO N460-N780 & E4 & W4 & 2 & E1 & W1 & E2 & W2 & $\mathbf{N}$ & 0 & \\
\hline 41 & W170 S1950-S2180 & E2 & W2 & 1 & E1 & W2 & E1 & W1 & $\mathbf{N}$ & 0 & \\
\hline 42 & W170 S1300-S1600 & E1 & wi & 1 & E1 & W1 & E1 & $W_{1}$ & $\mathbf{N}$ & 0 & \\
\hline 42 & W170 S1600-S1950 & E1 & W.1 & 1 & E1 & $W_{1}$ & E2 & W1 & $\mathbf{N}$ & 0 & \\
\hline
\end{tabular}


Table 2-2. Underground Inspection Survey Summary (Page 2 of 3)

$\begin{array}{cccccccc}\text { Zone } & \text { Area } & \text { Roof } & \text { Vert. } & \text { Ribs } & \text { Floor } & \text { Increased EEP/OBS Long-Term } \\ \text { Number } & \text { Description } & \text { Assessment } & \text { Fract. } & \text { Assessment } & \text { Assessment } & \text { Closure Assessment Projection } \\ & & & & & \text { Rate } & \end{array}$

\begin{tabular}{|c|c|c|c|c|c|c|c|c|c|c|}
\hline 43 & W170 $\$ 1000-\$ 1300$ & E1 & W1 & 1 & E1 & W1 & $E 1$ & W1 & $\mathrm{Y}$ & 0 \\
\hline 43 & W170 S700-S1000 & E1 & W1 & 1 & E1 & W1 & $\mathrm{E} 1$ & $W_{1}$ & $Y$ & 1 \\
\hline 44 & W170 S90-S700 & E1 & $W_{1}$ & 1 & E1 & $W_{1}$ & E2 & W2 & $Y$ & 0 \\
\hline 45 & W170/N150 S90/E0 & E1 & W1 & 1 & E1 & $W_{1}$ & E1 & W1 & $N$ & 0 \\
\hline 46 & S2180 E140-E300 & N1 & s1 & 1 & N1 & S1 & N1 & s1 & $N$ & 0 \\
\hline 47 & S2180 W30-E140 & N2 & S2 & 1 & N1 & S1 & N1 & S1 & $N$ & 0 \\
\hline 47 & $S 2180$ W30-W170 & N2 & S2 & 1 & N1 & $\mathrm{S1}$ & N1 & S1 & $N$ & 0 \\
\hline 48 & S1950 W30-E140 & N1 & S1 & 1 & N1 & S2 & N1 & S1 & $\mathbf{N}$ & 0 \\
\hline 48 & S1950 W30-W170 & N1 & S1 & 1 & N1 & S1 & N1 & S1 & $\mathbf{N}$ & 0 \\
\hline 49 & S1600 E140-E300 & N1 & S1 & 1 & N1 & S2 & N1 & $S 1$ & $N$ & 0 \\
\hline 50 & S1600 E140-W30 & N1 & S1 & 1 & N2 & S2 & N1 & $\mathrm{S1}$ & $\mathbf{N}$ & 0 \\
\hline 51 & $S 1600$ W30-W170 & N1 & S2 & 1 & N1 & S1 & N2 & S1 & $\mathbf{N}$ & 0 \\
\hline 52 & S1300 E140-E300 & N1 & S1 & 1 & N1 & S1 & Ni & S1 & $\mathbf{N}$ & 0 \\
\hline 53 & S1300 E140-W30 & N1 & S1 & 1 & N1 & S1 & N1 & $\mathrm{S1}$ & $\mathbf{N}$ & 0 \\
\hline 54 & $S 1300$ W30-W170 & N2 & S3 & 2 & N1 & S1 & N1 & s1 & $\mathbf{N}$ & 0 \\
\hline 55 & $\$ 1000 \mathrm{E} 140-\mathrm{E} 300$ & N1 & S1 & 1 & N1 & S1 & $\mathrm{N} 1$ & S1 & $N$ & 0 \\
\hline 56 & S1000 E140-W30 & N2 & S1 & 1 & N1 & S1 & N1 & S1 & $Y$ & 0 \\
\hline 57 & S1000 W30-W170 & N2 & S1 & 1 & N2 & S2 & N1 & $S 1$ & $N$ & 0 \\
\hline 58 & S700 E140-E300 & N1 & $\mathbf{S 1}$ & 1 & N1 & S1 & N1 & S2 & $N$ & 1 \\
\hline 59 & S700 E140-W30 & N1 & S1 & 1 & $N_{1}$ & S1 & N1 & S1 & $N$ & 2 \\
\hline 60 & S700 W30-W170 & N2 & S2 & 1 & N1 & S1 & $\mathrm{N} 1$ & $\mathrm{~S} 2$ & $\mathbf{N}$ & 0 \\
\hline 61 & S400 E140-E300 & N1 & S1 & 1 & N1 & $\mathbf{S 1}$ & N1 & $\mathbf{S 1}$ & $N$ & 0 \\
\hline 62 & WASTE SHAFT STATION & N1 & S1 & 1 & N1 & S1 & N1 & $\mathbf{S 1}$ & $\mathbf{N}$ & 1 \\
\hline 63 & S90 E140-E300 & N1 & S1 & 1 & N1 & $\$ 1$ & N1 & $S 1$ & $N$ & 0 \\
\hline 64 & S90 EO-E140 & N1 & S1 & 1 & N2 & \$2 & N1 & $\mathbf{s 1}$ & $N$ & 0 \\
\hline 65 & S90 W30-W170 & N1 & s1 & 1 & N1 & S1 & N1 & $\mathrm{S1}$ & $N$ & 0 \\
\hline 66 & S90 W170-Als & NA & S1 & 1 & N1 & S1 & N1 & $S 1$ & $Y$ & 0 \\
\hline 67 & S9O AIS-Q & N1 & S1 & 1 & N1 & S2 & N1 & S1 & $Y$ & 0 \\
\hline 68 & Q ALCOVE & N1 & S1 & 1 & $\mathrm{~N} 2$ & S2 & N1 & S1 & $N$ & 0 \\
\hline 70 & AIS STATION@SHAFT & E1 & W1 & 2 & E1 & W1 & E1 & W1 & $N$ & 0 \\
\hline 70 & AIS STATION LOW BRWS & E1 & $W_{1}$ & 1 & E1 & W1 & E2 & W1 & $\mathbf{N}$ & 0 \\
\hline 71 & ROOM V & N1 & S1 & 1 & N1 & S2 & $\mathrm{N} 1$ & S1 & $N$ & 0 \\
\hline 72 & AIS ACCESS N215 & N2 & S2 & 1 & N1 & s1 & N2 & S2 & $\mathbf{Y}$ & 1 \\
\hline 73 & N300 OE-WEST & N3 & S3 & 1 & N1 & S1 & N2 & S1 & $Y$ & 1 \\
\hline 74 & E140 N460 ALCOVE & N1 & S1 & 1 & N1 & S1 & N1 & S1 & $\mathbf{N}$ & 0 \\
\hline 75 & N460 E0-E140 & N1 & S1 & 1 & N1 & S1 & N1 & S1 & $\mathbf{N}$ & 1 \\
\hline 76 & E140 N780 ALCOVE & N1 & S1 & 1 & N1 & S1 & N2 & S1 & $\mathrm{N}$ & 0 \\
\hline 77 & N780 E0-E140 SHOP & N1 & S1 & 1 & N1 & S1 & N1 & S1 & $\mathbf{N}$ & 1 \\
\hline 111 & EO N620 ALCOVE & N1 & S1 & 1 & N1 & S1 & N1 & S1 & $N$ & 0 \\
\hline 112 & W170 CORE STORAGE & N1 & S1 & 1 & N1 & S1 & N1 & 51 & $\mathbf{Y}$ & 0 \\
\hline 113 & EO N150 OVERCAST & N1 & S1 & 1 & N1 & S1 & NA & NA & $N$ & 0 \\
\hline 114 & N150 OVERCAST-E140 & Nt & S1 & 1 & N1 & S1 & N1 & S1 & $\mathbf{N}$ & 0 \\
\hline
\end{tabular}




\section{Table 2-2. Underground Inspection Survey Summary (Page 3 of 3)}

Notes: All zones were assessed in October 1996.

The roof is assessed on a scale from 1 to 5 , " 1 " being no low-angle fracturing noted, and " 5 " being low-angle fracturing extending the full length of the zone, with separation or pull away in evidence.

Vertical fracturing is rated from 1 to 5 , with " 1 " being none observed, and " 5 " being close bolt-to-bolt fracturing. 0 indicates that no information is currently available.

Ribs are assessed on a scale of 1 to 3 , with " 1 " being good intact ribs, and " 3 " showing spalling or sloughing of the surface.

The floor is assessed on a scale of 1 to 3 , with " 1 " being a good intact floor with no fracturing or floor heave present. A " 3 " represents a badly fractured or heaving floor.

Under the heading "Increased Closure Rate," a " $Y$ " indicates that the measured closure rate at any convergence point within the area has increased more than 5 percent for the annual period ending October 31, 1996, as compared to the annual period ending October 31, 1995.

Under the heading "EEP/OBS Assessment," a "0" indicates no borehole data are available.

\begin{tabular}{|c|c|c|c|c|}
\hline \multirow[t]{3}{*}{ Key: } & $N$ & - North half of zone & $E$ & - East half of zone \\
\hline & s & - South half of zone & W & - West half of zone \\
\hline & N/A & - Not applicable or not available & & \\
\hline
\end{tabular}




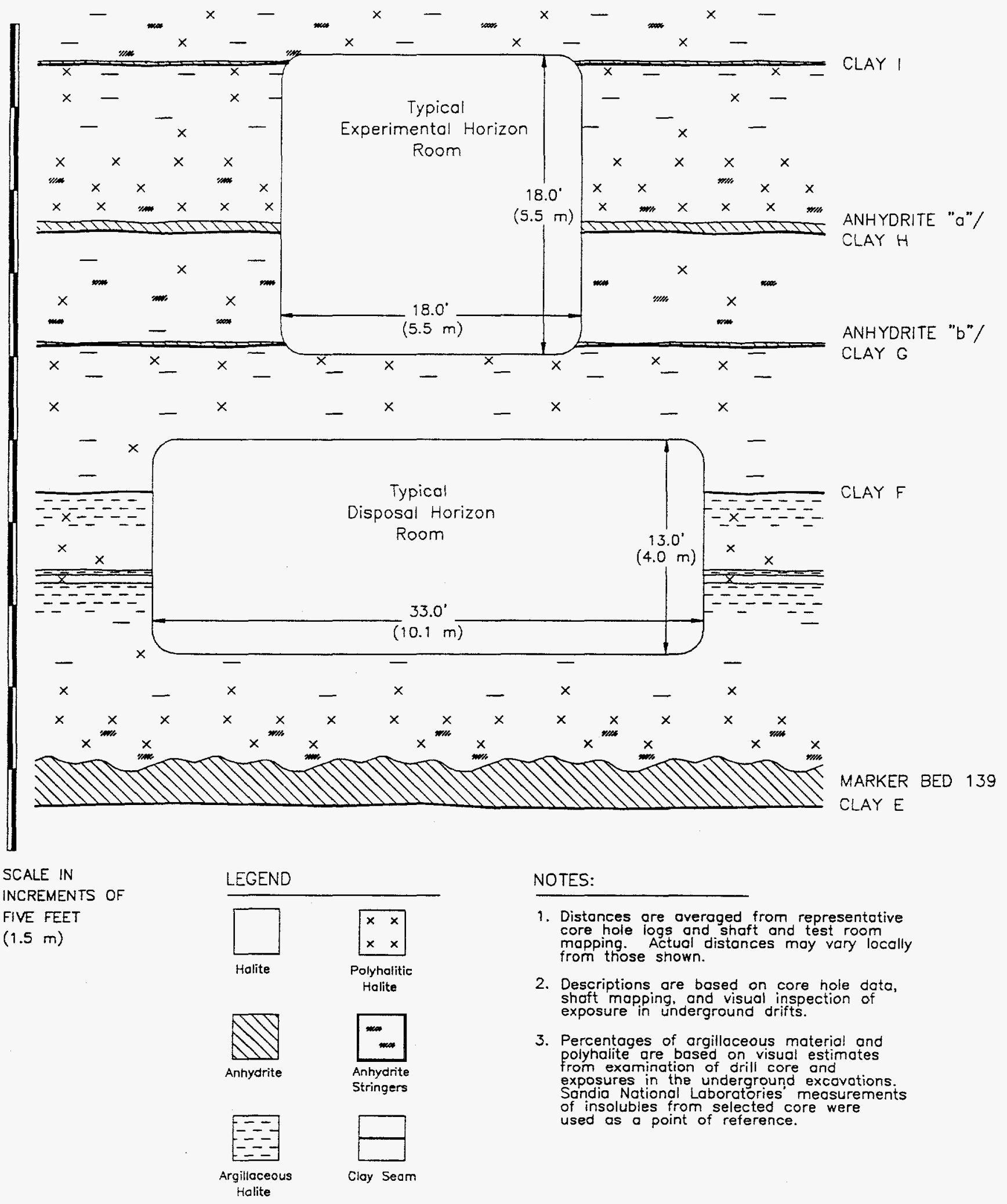

Figure 2-2. Typical Stratigraphy at the WIPP Facility Horizon 


\section{Disposal Horizon}

The stratigraphic location of the disposal rooms is referred to as the disposal horizon. Over 90 percent of the underground openings are located within this horizon, and all ground control projections presented in this AGCOP are related to this level. A 20-inch-thick to 32-inch-thick persistent bed of anhydrite, identified as Marker Bed 139 (MB 139), lies about 5 feet below the floor throughout the disposal horizon. Lateral variability in composition and thickness exists within this anhydrite bed at both repository and regional scales. MB 139 is underlain by clay $E$.

In relationship to the typical disposal room with a 13-foot excavation height, Anhydrite "a" is located approximately 13 feet above the roof and is underlain by clay $\mathrm{H}$, while Anhydrite " $b$ " is located approximately 6.5 feet above the roof and is underlain by clay $G$. Clay $F$ is found just below the roof elevation in most excavations. Anhydrite "a" is approximately 8.5 inches thick, and Anhydrite " $b$ " is approximately 2.5 inches thick. The clay seams associated with the anhydrite layers are approximately 0.25 inch thick. Clay $F$ is not a well-defined seam but is laterally discontinuous within the halite. The clay layers provide a surface along which slip can occur; whereas, the anhydrite layers are stiff units that do not creep. In addition, the undulating top of Marker Bed 139 resists shear movement along the interface with the overlying salt [U.S. Department of Energy, 1993a].

\subsection{Geometry}

The geometries or cross-sectional areas of the various drifts, rooms, and alcoves in the underground differ, primarily as a function of use. Most of the underground openings are rectangular in shape. The geometry of an opening, as well as the layout and geometry of surrounding excavations, plays a role in the stability of that area. The opening dimensions of each zone are given in Table 2-1. The geometries listed are generally "as mined" and will vary slightly because of original mining tolerance, closure, and maintenance activities such as scaling and milling. Major changes to geometry, such as the roof beam removal in the East-140 Drift, are represented in annual updates to the tables.

A beam of salt is formed between the roof of an excavation and the nearest anhydrite or clay seam above it. The thickness of the beam is determined by the height and stratigraphic location of the excavation. The structural relationship between the thickness of this beam and its width, its width being equal to the opening width, was 
evaluated. In general, the thinner and wider a beam is, the less structurally competent it is.

Historical geotechnical data on the stability of openings of various geometries are being evaluated. It may be possible to mine some excavations to geometries that enhance their stability. However, localized geologic conditions will always be a factor in opening stability, and the future use of an area must be considered when designing its geometry.

\subsection{Operational Use}

Long-term ground control plans are related to the operational use of specific areas. When designing a ground control system, the physical access requirements, as well as the geomechanical properties of an area, must be considered. Table 2-1 lists the current use of each ground control zone. Some forms of ground control may not be practical in certain areas, such as shops or offices. The projected life of an area is also directly related to its use. Some areas may have to be supported for only a few years; other areas may require support for decades. In areas that require support for long periods of time, for example, the life of the facility; the support system will probably undergo several changes during that time.

\subsection{Excavation Age}

Excavation of the underground facility began in 1982, and over 60 percent of the existing openings were completed by the end of 1984. The average age of an opening is 11.5 years, with some openings being over 14 years old. Table 2-1 lists the current age of each ground control area. The age of an excavation is important with respect to ground control because of the amount of deformation that has already occurred and the amount that is anticipated to occur during its projected life.

Some underground openings at WIPP must remain accessible for long periods of time: up to an anticipated life of 50 years for some main entries. The 50-year life is based on 14 years since excavation, 1 year until receipt of waste, and a 35-year operational life after receipt of waste. With this time frame in mind, support systems must be designed to accommodate creep-related deformation and to support the beam (if it becomes detached). The age of an excavation at the time a support system is installed and the age of systems in place are factors that are considered when evaluating the long-term effectiveness of those systems. 


\subsection{Projected Life}

Based primarily on its operational use and projections for receipt of waste presented in the WIPP Disposal Decision Plan (DDP), an estimated life was assigned to each zone. The DDP that is included in the Panel 1 Utilization Plan provides a time line to receipt of waste. This time line was used in formulating the projected estimated life of related zones [Westinghouse Electric Corporation, 1994]. Three categories, short-term, intermediate, and long-term, were established for this purpose. These projections are an additional tool used in the ground control selection process. The criteria for these designations are:

- Short-Term (ST) - A projected life of less than 10 years. This designation includes waste disposal rooms.

- Intermediate (I) - A projected life of 10 to 15 years. Special use areas such as maintenance shops are included here. Shops will be required for the life of the facility, but because of creep-related closure, it is assumed that they may be relocated periodically.

- Long-Term (LT) - A projected life of up to 40 years or the life of the facility. This designation covers all areas critical to the long-term operation of the facility. Shaft stations, main ventilation drifts, and main access and haulage routes fall in this category.

These projections use the excavation date as a start time. If the current use of an area changes or receipt of waste is delayed significantly, designations for specific zones may be adjusted (e.g., a short-term area may change to an intermediate). Areas closed to access are designated as such and have no projected life. The closed areas could be reopened; but this is not anticipated, with the exception of East-140 south of South-2180. These projections are unofficial and are used solely for the purposes set forth in this plan, primarily ground control planning. Table 2-1 lists the projected life of each zone.

\subsection{Ground Conditions}

Because salt is a rock that creeps when subjected to load, once an opening is made, a continuous process of deformation and associated fracturing is initiated that is the primary parameter affecting the condition of an excavation. The ground condition in turn is the primary parameter dictating the type of ground control measures that will be employed. 


\subsubsection{General Roof Beam Failure Mechanisms and Patterns}

Roof beam conditions in general follow a predictable path of deterioration over time with the degree of degradation varying widely throughout the facility. Many areas have remained very stable since their excavation, while low-angle fracturing and bed separation are observed in other areas. These fractures develop from the ribs upward over the center of the room. In areas where this process is advanced, fracturing on one side typically predominates, and a cantilever forms and must be supported to prevent catastrophic failure, such as a roof fall.

\section{Time-Dependent Degradation}

A virgin stress field exists in the rock prior to mining. When an excavation is made, that stress field is disturbed, creating differential stresses that result in creep, and as a result, closure of the room. With time, the stresses close to the excavation are relieved by deformation of the salt into the excavation. Differential stresses and related strains develop on each side of the strata interfaces (clay and anhydrite seams). They may eventually lead to slippage at these contacts and eventually to bed separation. Because the clay seams do not provide much, if any, shear resistance, the salt between clay $G$ in the roof and clay $E$ below the floor moves horizontally toward the excavation faster than the adjacent layers. This movement concentrates the stresses on those portions of the layer remaining after the excavation (i.e., the portion between the roof and clay G, called the roof beam, and the portion between the floor and clay $E$, called the floor beam). The stresses in the roof and floor beams are manifestations of the separate processes of bending, dilation, and shearing, which all take place at all times. Any one of these processes may predominate at any particular time. The concentration of stresses and strain in the roof and floor beams may reach values high enough to cause (or at least initiate) failure. This failure can be, and commonly is, in the form of low-angle fractures. Low-angle fractures as discussed in this text refers to fractures initiating at or near the rib-roof intersection and propagating upward at an angle less than 45 degrees. These fractures terminate when they reach a discontinuity in the stratigraphy, such as a clay seam. Once the low-angle fractures develop, roof movements in an unsupported excavation are increasingly associated more with gravity effects than with salt-creep-driven effects. A detailed description of the failure mechanisms involved can be found in U.S. Department of Energy [1993b].

Detailed observation and mapping of exposed fracture systems during beam removal activities have led to the conclusion that the formation of the low-angle fractures is 
more complex than simple compressive failure. In addition to the compressive (horizontal) forces acting on the roof beam, gravity also plays a role. Gravity and creep produces a downward bowing of the beam most prominent near the center of the opening. The sagging of the beam coupled with the horizontal forces creates a horizontal or sub-horizontal en-echelon type fracture sequence (i.e., the initial fractures are oriented to the left or right of each other like a series of steps). These fractures ultimately connect to form the low-angle fractures. Figure 2-3 illustrates this process.

\section{Roof Beam Failure Patterns}

The fracture mechanisms discussed above have proven to be consistent in areas experiencing advanced degradation of the roof beam. Roof falls in Site and Preliminary Design Validation (SPDV) Rooms 1 and 2 indicate that a detached section of a roof beam will be somewhat of a wedge shape. Observations of the fracture patterns exposed during the beam removal process in the East-140 Drift confirm this mode of failure. Knowledge of the type of failure that can be expected in the roof beam aids in the design of ground support systems. For example, support systems designed to support the entire cross-sectional area of the roof beam are considered conservative based on a wedge-type failure. Figures 2-4 through 2-7 illustrate the types of fracture patterns and bolt loading that are typically observed in the roof beam. Figure 2-4 shows that by ending the resin column approximately 1 foot above the clay seam as opposed to flush with the seam, it is anticipated that the bolts will be subjected to a less severe bend as a result of offset. The offset of the bolts as shown in the figures is exaggerated for illustrative purposes.

\subsubsection{Panel 1}

The ground conditions in Panel 1 follow a pattern similar to what was observed in the areas of the East-140 Drift where the beam was removed. Low-angle fractures of varying degrees are observed in all areas. The installation of rockbolts has, in many instances, resulted in the thin edge of the wedge associated with a cantilevered beam breaking with an associated vertical fracture that runs roughly parallel to the rib.

Table 2-3 depicts the total vertical and horizontal convergence for the Panel 1 rooms since their dates of excavation. As is illustrated in the table, total vertical convergence is well over 2 feet in some areas of the panel and horizontal convergence is over 19 inches. Because ground control measures have little or no effect on the creep process, convergence of this type will continue unabated until the openings are completely closed. 
PHASE 1

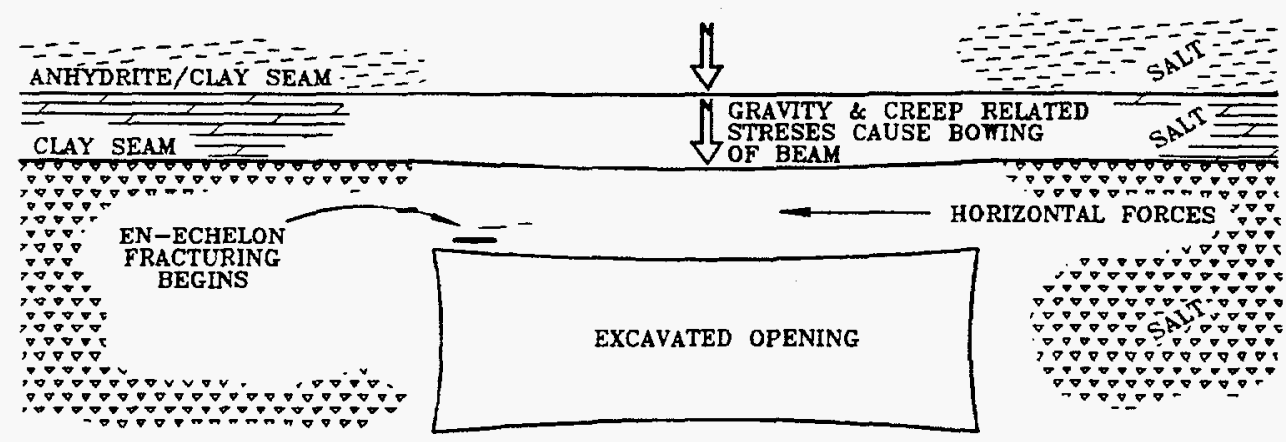

PHASE 2

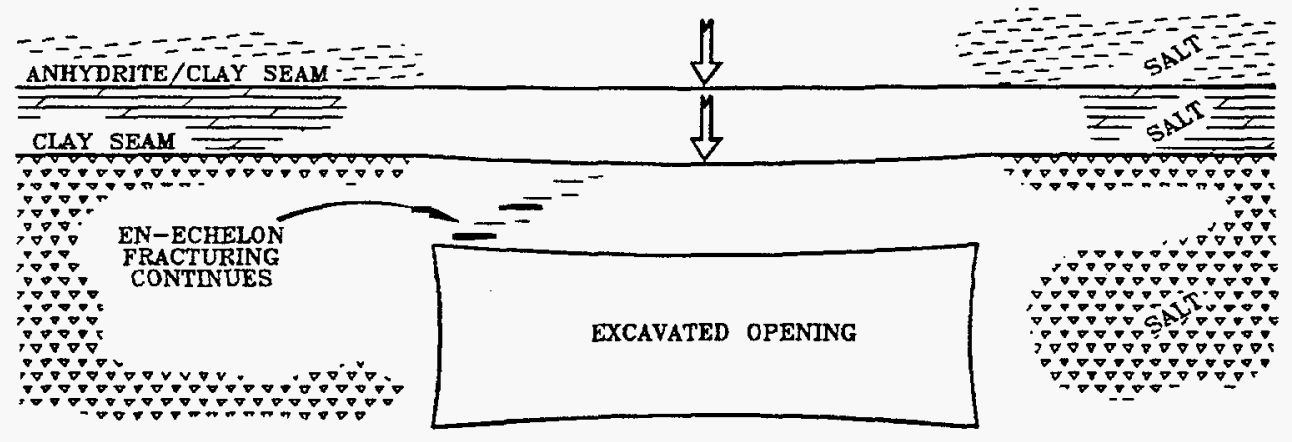

PHASE 3

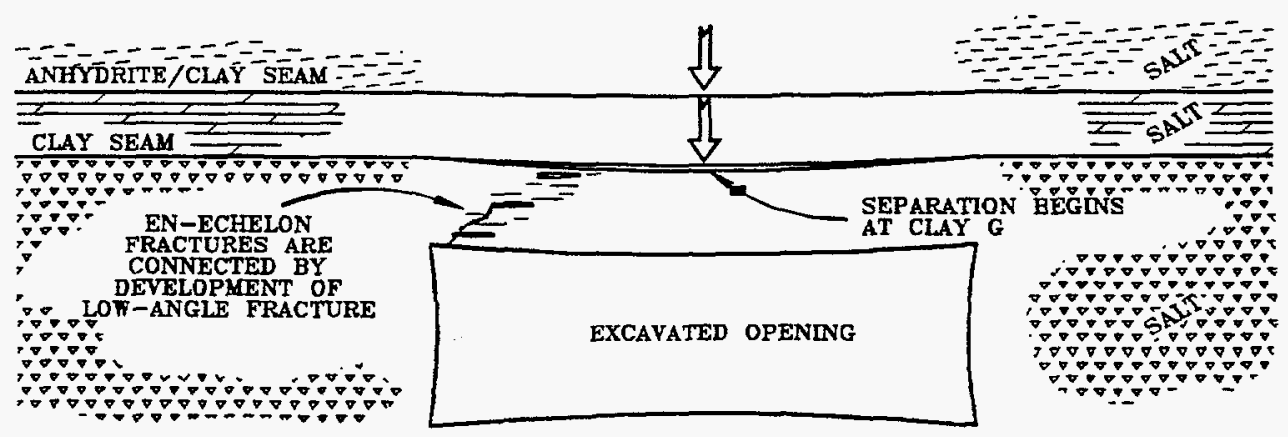

$\underline{\text { PHASE } 4}$

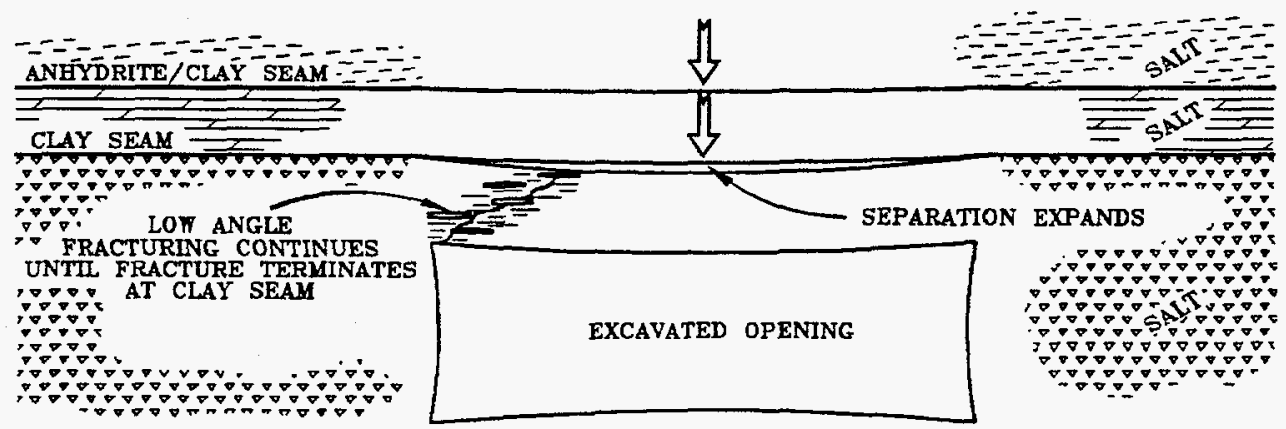

Figure 2-3. Generalized Fracture Development Sequence 


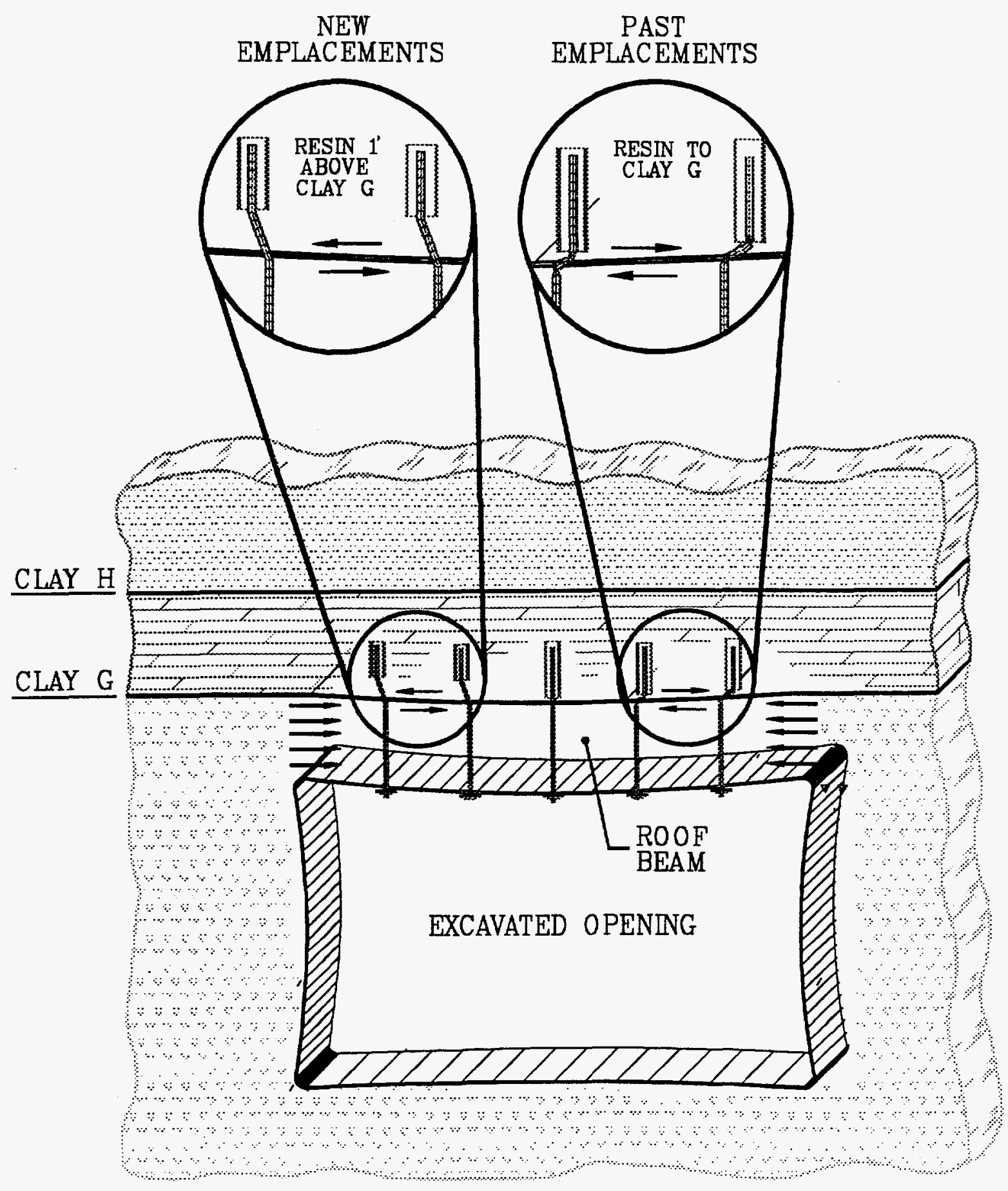

Figure 2-4. Roof Beam Offsetting and Related Bolt Loading 


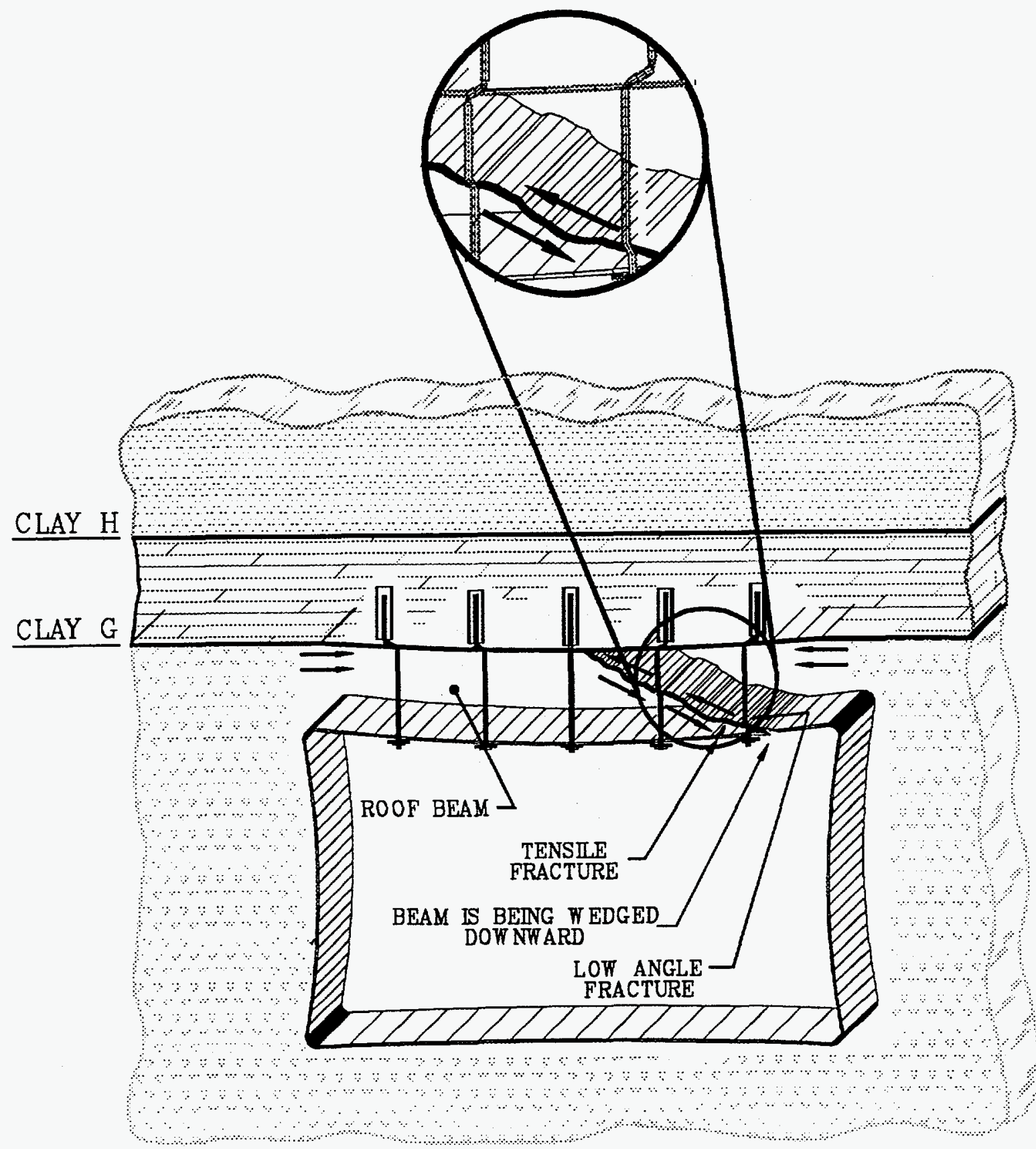

Figure 2-5. Typical Roof Beam Fracturing 


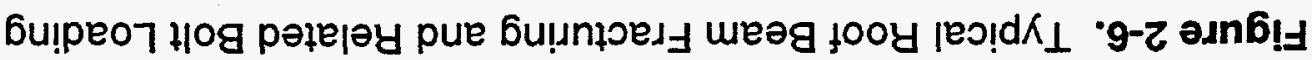

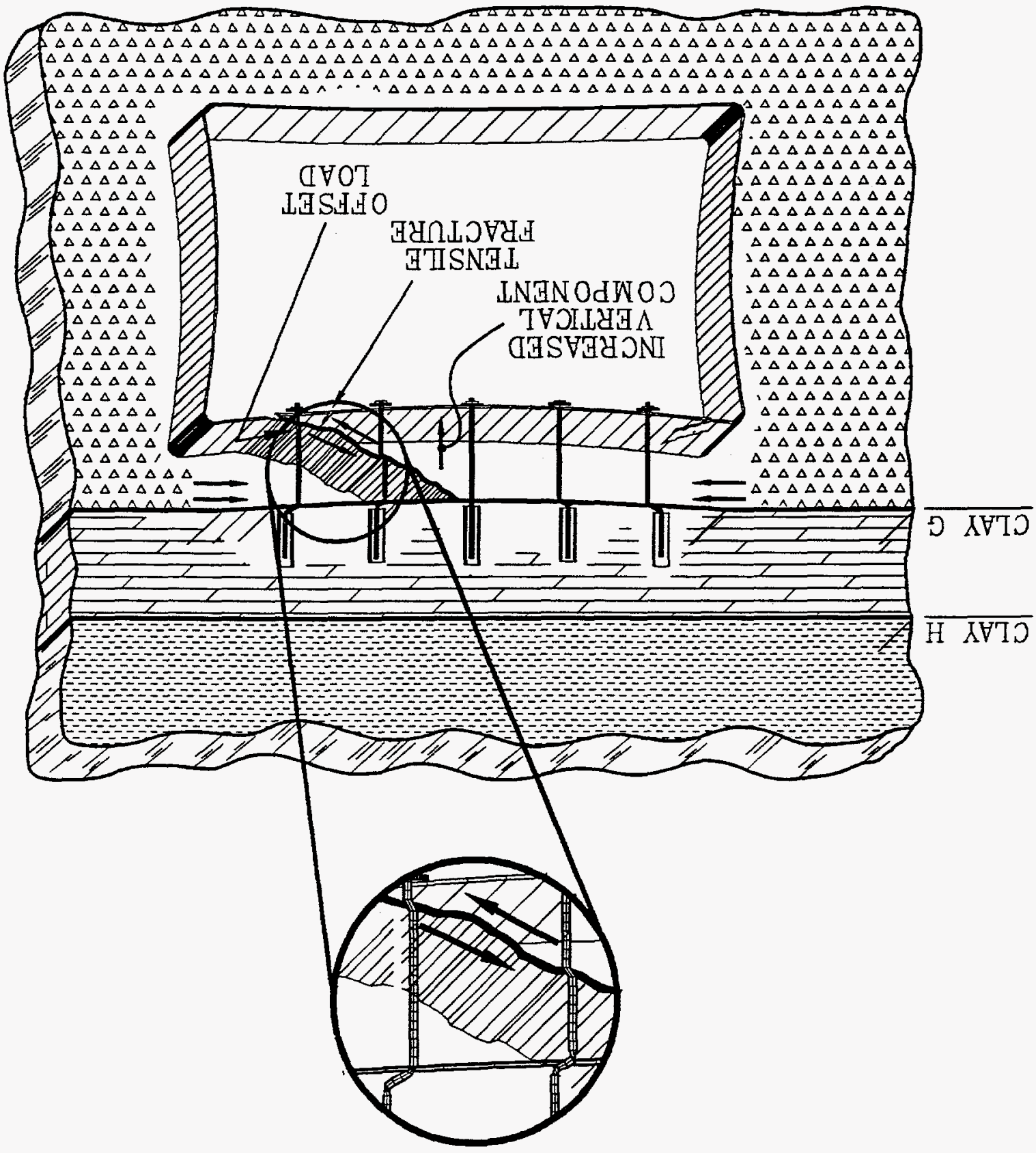




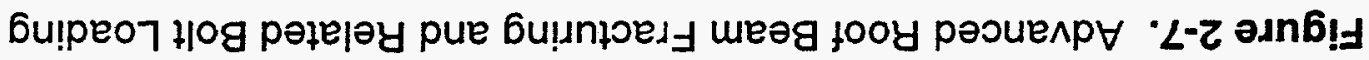

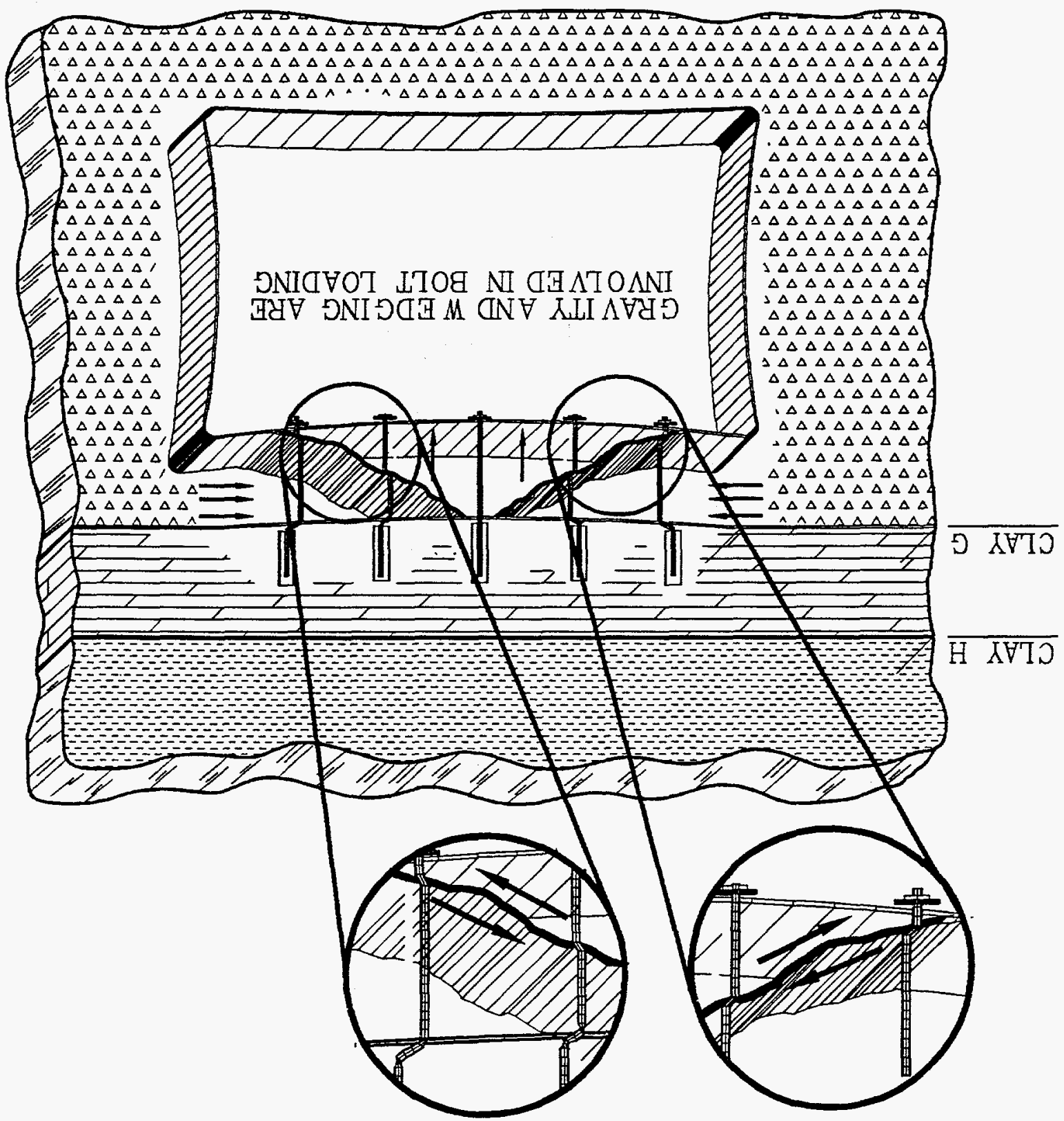




\section{Table 2-3. Panel 1 - Total Vertical and Horizontal Closure}

\begin{tabular}{|c|c|c|c|c|}
\hline Room & $\begin{array}{c}\text { Date of Excavation at } \\
\text { Instrument }\end{array}$ & $\begin{array}{c}\text { Excavation } \\
\text { Completed to Final } \\
\text { Dimensions }\end{array}$ & $\begin{array}{c}\text { Total Vertical Closure as } \\
\text { of Reporting Period } \\
\text { (in) }\end{array}$ & $\begin{array}{c}\text { Total Horizontal Closure } \\
\text { as of Reporting } \\
\text { Period } \\
\text { (in) }\end{array}$ \\
\hline Room 1 & June 1986 & August 1986 & 31.66 & 19.05 \\
\hline Room 2 & January 1987 & March 1988 & 25.46 & 15.86 \\
\hline Room 3 & February 1987 & March 1988 & 27.24 & 19.83 \\
\hline Room 4 & February 1988 & March 1988 & 24.14 & 16.17 \\
\hline Room 5 & February 1988 & March 1988 & 23.32 & 16.70 \\
\hline Room 6 & February 1988 & May 1988 & 24.99 & 15.08 \\
\hline Room 7 & March 1988 & March 1988 & 25.32 & 14.96 \\
\hline
\end{tabular}

(a) Data taken from Geotechnical Analysis Report for Juby 1994 - June 1995 [U.S. Department of Energy, 1996].

Data indicate that the majority of Panel 1 excavations show an expansion rate of the roof beam from the exposed surface to Anhydrite " $b$ " to be fairly constant at about 0.5 inch per year. Roof beam expansion rates in more active areas of the panel have ranged up to 1.8 inches per year. The more active areas have all had supplemental ground support installed.

\subsection{Support System Conditions}

\subsubsection{General}

Excluding Panel 1, the ground support in most of the accessible areas underground consists of mechanical-anchor rockbolts anchored above the first clay seam. Other than bolts that have noticeably failed, it is difficult if not impossible to tell what condition the remaining bolts are in. However, based on measured deformation and observed offset since their installation date, it can be surmised that a significant number of the mechanical-anchor rockbolts are probably in material yield, are experiencing anchor slippage, or have failed. In the northernmost areas of the East- 0 Drift and the East-140 Drift where rebolting with threaded-bar bolts and yielding cable bolts was performed, the bolts are relatively new and should be in good condition.

\subsubsection{Panel 1}

Various support systems have been installed in Panel 1 over a several-year time span. The types of systems and their installation dates are specified in Section 5.1 of this 
report. As in the other areas of the facility, it is difficult if not impossible to tell what condition the in-place bolts are in. However, based on measured deformation and observed offset since their installation date, a significant number of the mechanicalanchor rockbolts installed in Rooms 1 through 6 are probably in material yield, are experiencing anchor slippage, or have failed. Since the mechanical-anchor bolts installed in Room 7, South-1600, and South-1950 do not penetrate the first clay seam, they have not been exposed to the same degree of lateral and axial deformation as the 10 -foot bolts. Because of this, they may be in relatively good condition.

With the exception of parts of Rooms 1 and 2, the entire panel has been rebolted or bolting is in progress with resin-anchored, threaded-bar bolts. The condition of these bolts will vary dependent on their date of installation, location, and method of installation. Excluding the mechanical bolts, the threaded bar installed in Room 1 is the oldest system in place in the panel. Although these bolts are being detensioned manually, a few bolt failures, apparently related to offset-induced lateral loading, have been observed. It is known that the vast majority of the bolts are intact evidenced by the fact that they continue to load and require detensioning.

The remaining threaded-bar systems in the panel are relatively young and should be in good condition. The rockbolt boreholes were oversized below the clay seam to allow for a larger amount of offset prior to the bolts being affected by lateral loading. In addition, the latest installations have the resin column terminated approximately 1 foot above the clay seam to allow for a less severe bend in the bolts once lateral loading begins.

Given the baseline schedule, it is clear that bolts will fail in increasing numbers and require replacement as the panel ages. If as many as 25 percent of the pattern bolts in the panel fail prior to panel closure and given the sequence for filling the panel, it is projected that approximately 225 bolts will need to be replaced by Year 2002 . Any schedule acceleration helps obviate this problem, while schedule delays exacerbate the problem.

\subsection{MONITORING AND EVALUATION}

The assessment and evaluation of the condition of WIPP excavations is an interactive, continuing process involving a wide variety of data. These evaluations can be as simple as the required daily visual site checks by personnel working in an area or as complex as the expert review of Room 1, Panel 1 [U.S. Department of Energy, 1991a]. 
The Geotechnical Engineering group gathers and evaluates data from various sources on a daily and weekly basis. A bimonthly underground geotechnical assessment report is prepared, as is the annual Geotechnical Analysis Report. An in-depth evaluation of all of the accessible underground is performed on an annual basis as part of the preparation of this plan. These evaluations are based on visual observations by Geotechnical Engineering personnel, analyses of instrumentation data, observation borehole data, and rockbolt failure patterns. Roof stability is considered to be of primary interest, while rib and floor stability is secondary.

Remote monitoring of geotechnical conditions and ground support systems in selected locations of the closed areas north of North-780 is also being performed. The monitoring continues to assist in evaluation of systems as they age and trend toward failure. It is intended that data from these zones will provide predictive information on ground falls in areas with installed roof support systems.

\subsection{Geotechnical Evaluation Process}

One of the more difficult aspects of ground control is determining and evaluating the criteria that dictate when ground control actions should be initiated. The identification of instabilities is critical to maintaining a safe underground environment. Ground control can be expensive and, in some instances, ground control measures can actually have an adverse effect on the in situ conditions (e.g., the breakup of a beam associated with installation of rigid bolts). Therefore, it is prudent to be as rigorous as possible in determining when to initiate ground control actions and what those actions should be. The process followed at WIPP includes evaluation of three general categories of information. These categories include:

- Collection and analyses of geomechanical instrumentation data.

- Evaluation of the performance of installed ground support systems.

- Evaluation of physical observations.

Each category is evaluated independently and comparatively to the other categories. With respect to Panel 1, waste emplacement schedules must also be considered for logistic purposes. Criteria for corrective action are continually reevaluated and reassessed based on total performance to date. Actions taken are based on these analyses and planned use of the excavation. 
Collection and analyses of geomechanical instrumentation data

Instrumentation data provide quantitative information on rock movement in and around an opening. Convergence and extensometer data are collected on a continuing basis. This information is plotted as displacement versus time and as rate of displacement versus time. These data are analyzed concentrating on trends in rates and changes in patterns as predictors of instability. For example, long-term data may indicate a consistent closure rate in a particular area of approximately 0.5 inch per year. A significant acceleration in this rate may be a warning sign of possible instability.

\section{Evaluation of the performance of installed ground support systems}

Bolt failures alone do not necessarily indicate an unstable situation. Because of the deformation process associated with creep, bolts are going to fail. The age of the inplace ground support, as well as the roof beam expansion rates and relative stratigraphic offset rates, must be considered when evaluating system performance. Knowing the mechanical properties of support system components and experience with the systems in the WIPP environment enables the engineer to predict how the system should perform under specific conditions. A warning flag is raised when a ground support system is performing in a manner inconsistent with what is expected.

\section{Evaluation of phvsical observations}

Physical observations generally include surface fractures, fractures within boreholes, offset in boreholes, spalling, and any other visually detectable behavior of the ground condition. Similar to the other data, anomalous behavior, such as accelerations in fracture development or increased slabbing, is an indicator of potential instability.

\subsection{Surface Observations by Geotechnical Engineering}

A two-person team from Geotechnical Engineering completed a visual assessment of the underground facility during the period from October 8, 1996, through October 31 , 1996. The conditions of the roof, ribs, and floor were assessed in each ground control zone and were graded on a scale basis. For the purpose of these evaluations, lower numbers represent better conditions (i.e., "1" is good). The roof was evaluated with respect to low-angle fractures, scaling, and longitudinal or transverse vertical fractures, and then graded on a scale of 1 to 5 . The ribs were evaluated on a scale of 1 to 3 based on their general condition, and it was noted if they had been mechanically 
scaled. The floor was evaluated with respect to heaving and fracturing and graded on a scale of 1 to 3. It was also noted if the floor had been milled. As a general rule, scaling and milling activities remove small amounts of ground and are unlikely to have a significant effect on closure rates and the overall stability of the area.

For evaluation of the ribs, floor, and low-angle fractures in the roof, each zone was divided in half down its length. If an area was north-south running, an assessment of the east side and the west side was performed. An east-west running zone was evaluated on its north and south sides. A summary of these evaluations is given in Table 2-2.

The roof of an area was taken as a whole with regard to longitudinal or vertical fractures. A few vertical fractures may be an indicator of advancing deterioration, but they do not constitute reason for immediate remediation. Areas containing vertical fractures are closely monitored both mechanically and visually. The type and extent of these fractures will affect the ground control system chosen for a given area. An area with only a few longitudinal fractures could probably be supported with a standard bolt system, whereas an area with extensive, connected fracturing suggesting a breakup of the beam might require a wire mesh or a cable mesh system. The ratings of each zone with respect to vertical fracturing are presented in Table 2-2.

Areas receiving higher numbers, based on the visual assessments, warrant closer monitoring. In these cases, the ground conditions are monitored more frequently, and additional instrumentation is installed when appropriate. At a minimum, areas with a long-term projection rating of "3" are formally reviewed quarterly, those with a "2" are reviewed semiannually, and those rated as " 1 " are reviewed annually. When it is determined that ground conditions have reached a point where a safety hazard could develop in the short term, mitigation actions are implemented.

\subsection{Observation Borehole Data}

Fracture development in the roof is related to the stress regime in the roof beam and is influenced by the shape and age of the excavation, local stratigraphy, nearby excavations, and mining method and sequence. Large strains associated with lateral movements in the roof can induce the fracturing in the roof that is frequently seen near the ribs. The presence of horizontal offsetting (visible in boreholes) confirms lateral movements in the roof beam. The results of borehole observations indicate that fracture development continues as the excavations age. 
- Guidance for design modifications and remedial actions.

- Data for interpreting the actual behavior of underground openings, in comparison with established design criteria.

- Data on which to base an accurate assessment of the mechanisms of deformation and fracturing that is taking place.

Geotechnical data collected from each specific ground control zone are evaluated to determine if conditions exist which would warrant closer attention or possibly immediate attention from a ground control standpoint. For the long term, roof expansion rates, along with the expected life of a zone, are important criteria to be considered when selecting ground control measures for that area.

Measurements of roof-to-floor and rib-to-rib closure are taken throughout the underground, both manually and remotely, on a routine basis. In addition to closure data, extensometer data are also collected. Extensometer data, combined with information from observation holes, allow the monitoring of separations at clay seams and within salt beams (beam expansion). A comparison of closure rates at convergence points for the current evaluation period and the last evaluation period was performed. An increase in the closure rate does not necessarily indicate a problem but draws attention to that area and provides an additional data point for evaluation. A summary of this assessment is presented in Table 2-2; a " $Y$ " (yes) indicates that the measured closure rate at any convergence point within the area has increased more than 5 percent for the annual period ending October 31, 1996, as compared to the annual period ending October 31, 1995.

\subsection{Rockbolt Failure Data}

Rockbolt failures are documented to have occurred throughout the underground facility. initially, many of the failures were bolt head failures, which may be attributed to installation problems. Currently, the majority of bolt failures appears to be associated with lateral movement at clay $\mathrm{G}$.

Observable rockbolt failures are reported and a database on failure locations and modes of failure is being maintained. Much has already been learned from the analysis of past bolt failures, and as the information base increases, so does our understanding of the failure mechanisms involved. Documentation and tracking of these failure patterns assist in long-term ground control planning by highlighting problem areas. This system also provides a means to identify trends of bolt failures that may be 
correlated with installation methods, geometry, mining sequence, and other variables. Figure 3-1 presents a graphical representation of rockbolt failures by type.

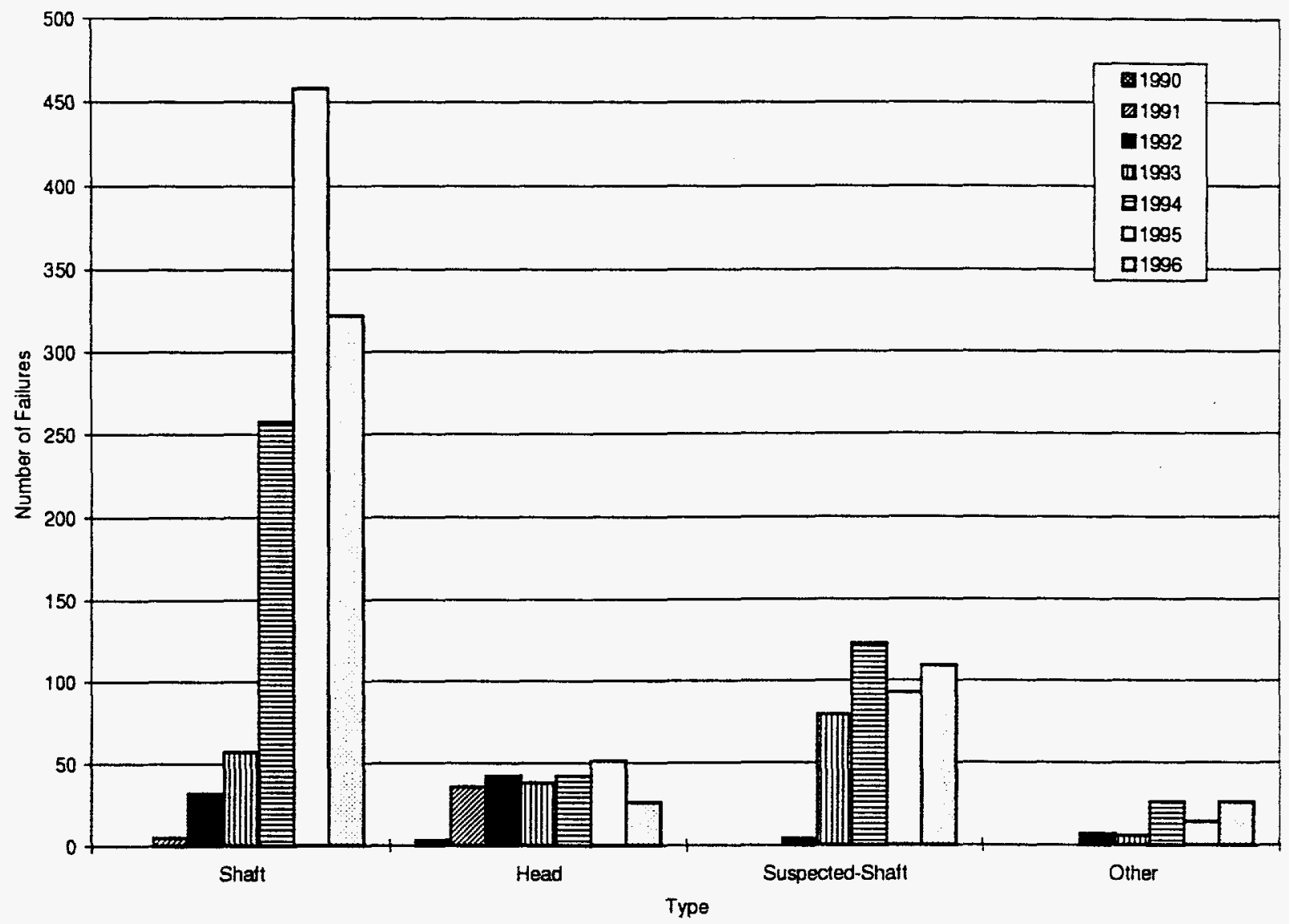

Figure 3-1. Rockbolt Failures by Type

In addition to tracking the rockbolt failures, a limited rockbolt failure investigation program was implemented. Several borehole camera surveys were performed in boreholes of failed bolts. One failed rockbolt was overcored to observe the condition of the remaining portion of the bolt located above the first clay seam. Observations from this investigation showed corrosion surfaces on the portion of the bolts remaining in the hole and salt creeping or flowing around the bolt shaft. A study was also initiated to address corrosion and loading effects on the premature failure of support system components. 


\section{Rockbolt Failure Mechanisms}

A consultant with expertise in evaluating material properties and related failure mechanisms was contracted to take an in-depth look at the rockbolts being used at WIPP and evaluate the effects of corrosion and its relationship to premature failures. The consultant reported that corrosion did not play a major role in the early failure of bolts. However, the mechanical cyclic stress loads the bolts are exposed to while they are under tensile load (that load sometimes being in excess of the bolts yield limit) result in fatigue failure.

A materials evaluation of the mechanical-anchor-type rockbolts and the threaded-bar rockbolts used at WIPP revealed that there was considerable variation in content; for example, carbon content, from bolt to bolt. The variability in content means that each bolt is going to respond differently, to some degree, to similar loading conditions.

\subsection{Ground Support System Monitoring}

Instrument monitoring of ground support systems has expanded significantly in the last few years. Table 3-1 presents a summary of the ground control system instrumentation that is currently in place in the facility.

\section{Table 3-1. Ground Control System Instrumentation}

\begin{tabular}{|c|c|c|c|c|c|c|c|c|c|}
\hline \multicolumn{2}{|c|}{ Rockbolt Load Colls } & \multicolumn{2}{|c|}{ Rockbolt Strain Gages } & \multicolumn{2}{|l|}{ Slipnuts } & \multicolumn{2}{|c|}{ Load indicators } & \multicolumn{2}{|l|}{ Joint Meters } \\
\hline Location & Qty. & Location & Oty. & Location & Oty. & Location & aty. & Location & Qty. \\
\hline Room 1, Panel 1 & 288 & Room 4, Panel 1 & 38 & Room 4, Panel 1 & 186 & Room 2, Pand 1 & 132 & Room 4, Panel 1 & 3 \\
\hline Room 2, Panel 1 & 136 & Room 7, Panel 1 & 46 & East 140 & 4 & Room 7, Panel 1 & 18 & Room 6, Panel 1 & 1 \\
\hline Room 4, Panel 1 & 10 & $E-140, N-600$ & 4 & Room D(a) & & E-O North & 1 & Room 7, Panel 1 & 2 \\
\hline Room 7, Paned I & 4 & $E-140, N-800$ & 8 & & & & & Waste Shaft Brow & 1 \\
\hline E-140, South & 6 & Salt Shaft Brow Cable & 1 & & & & & E0 & 2 \\
\hline E-140, North & 74 & & & & & & & AIS Brow & 2 \\
\hline EO Overcast & 2 & & & & & & & $E-300, S-1950 O C$ & 4 \\
\hline$E 0, N 335$ & 1 & & & & & & & & \\
\hline AIS Brow & 20 & & & & & & & & \\
\hline Waste Shaft Brow & 10 & & & & & & & & \\
\hline E-0 North 800 & 4 & & & & & & & & \\
\hline
\end{tabular}

(a) In a closed area being monitored remotely. 


\subsubsection{Load Cells}

Load cells provide a quantitative measurement of the axial load on individual rockbolts or individual components of a ground support system. Load cells are installed on cable systems used to support brow areas at the Waste Shaft and Air intake Shaft (AIS) Station areas and the East- 0 overcast. Load cells installed on rockbolts in Panel 1, Rooms 1 and 2, are used to indicate when detensioning will be performed. Load cells are also installed on rockbolt support systems in other areas of Panel 1, East-140, and in Room $D$ to monitor total load on specific components. Load cells included in yielding support systems, such as the yielding cable bolt installations, monitor whether the systems are performing as intended (e.g., yielding at their designed loads).

\subsubsection{Strain Gages}

The supplemental bolt patterns that were installed in Rooms 4 and 7 of Panel 1 and in Room D all incorporated systematic arrays of rockbolts equipped with strain gages. The strain gage data are providing a variety of valuable information, which includes:

- Areal effects of creep-related closure loading on the ground control system. If roof areas within an instrumented array are experiencing differing degrees of deterioration, that information can be compared to the strain rates being produced in the rockbolts.

- The ability to determine when the bolts reach their yield point.

- In areas where yielding systems or components are being evaluated, a comparison can be made between the yield point of the yielding component and the strain in the bolt.

- Strain gage data can be compared to load cell data.

\subsubsection{Yielding Components}

Yielding components such as slipnuts, load indicators, and yielding cable bolts, are incorporated into several of the ground support systems in the facility. The primary purpose of these components is generally to allow for the system to yield with the creep-related deformation of the formation. However, because the components are designed to yield within specific load ranges, they also serve the function of providing an indication of the load on the system. These components are visually monitored to determine the rate and degree of yield on the system. 
Recently, Titan load indicators designed to yield at 20,000 pounds were installed on the threaded-bar system in Room 2, Panel 1 . This system is being detensioned when bolt loads reach approximately 20,000 pounds. The yield range on the load indicators has decreased the frequency of the detensioning process from approximately once a month to approximately once a year.

\subsubsection{Joint Meters}

The joint meters used at WIPP serve primarily as a geotechnical tool (i.e., to monitor fracture growth). However, a couple of meters have been incorporated into cable mesh support systems to monitor strain in the cables.

\subsection{GROUND CONTROL OPTIONS}

The end objective of each ground control option is to provide safe, geotechnically stable access for personnel and equipment. The options available to meet this objective involve providing internal support (e.g., bolts of various kinds), external support (e.g., cribs), or removing the roof beam. Closure of selected areas is an administrative choice in lieu of ground control options. Simply stated, these options are:

- Support the ground

- Remove the ground

- Close the area.

When evaluating these options, the criteria by which each option is judged include: the degree of safety provided, the support capacity of the system, ease of implementation, "life expectancy" of both the system and the area in which it is being implemented, economics, and the impact of the chosen method on waste emplacement. It is important to appreciate the complexity of weighing these criteria relative to the differing conditions experienced throughout the facility. It is also important to understand that, although some of the options have no resource constraints, others may be difficult to implement at given times. (For example, it may be difficult to mine the roof beam in Panel 1 if both continuous mining machines are active in mining Panel 2.) This section describes the applications of various support systems that are being used, tested, or proposed for possible use at WIPP.

Because of the proactive ground control program now in place, an extensive database of information on ground control systems that are related directly to WIPP conditions is 
being developed. Monitoring of installed supplemental systems will continue to provide a greater base of information for ground control system selection. In addition to in situ testing, a laboratory testing program is in place to evaluate alternative options for ground support. Appendix A provides a summary of testing performed and an evaluation of in-place support systems.

\subsection{Internal and External Support Systems}

\section{Internal Support Systems}

All of the ground support in currently accessible areas of the underground is provided by internal systems. Internal systems offer the least interference with respect to operational concerns, such as waste handling activities. In general, internal support systems consist of pattern rockbolting.

The primary purpose of pattern bolting is to support the roof beam or, more accurately, a portion of the roof beam, should it become detached as a result of fracturing. A variety of rockbolt patterns exists throughout the underground at WIPP. The design of the patterns is based on the width of the opening and the thickness of the roof beam. The effect of rock creep-related deformation on rockbolts creates a problem that is not common to standard ground support design practice. Normally, a rockbolt pattern is designed such that the dead weight of the rock, with a factor of safety included, does not exceed the yield limit of the bolts. In rock such as halite, the mechanism of creeprelated closure may put the bolts into yield as soon as 1 year after installation, regardless of the number of bolts installed. This may happen sooner if bolts are installed immediately after excavation. The highest strain rates around a new opening are recorded immediately after the opening is excavated. These rates taper off to near steady state after approximately 1 year. Creep also produces an offset of the strata at the first clay seam above the roof. The clay seam, with no inherent strength, facilitates a separation between the roof beam and the strata above it. Different stress conditions above and below the seam produce differential strain rates, which results in an offset at the stratigraphic boundary. This offset eventually places a nonaxial load on the bolts that reduces their axial load capacity. The creep-related parameter in support system design complicates the selection process. In addition to the dead weight of the material to be supported, roof expansion rates, installation time with respect to excavation date, offset, and bolt load/elongation properties must be considered in any support design. 
DOE/WIPP 97-2222

\section{External Support Systems}

External ground control options have not ranked high when evaluating support systems for two primary reasons: (1) there is no evidence at this time that external systems offer substantial advantages over internal systems and (2) external systems are much more intrusive from an operational interference standpoint. The type of external support most often used in the mining industry is cribs. For cribs to be effective, they would need to be extensive, which would severely restrict storage area and operational maneuverability (i.e., it may not be operationally possible to place them where required). If cribs are used sparingly to support specific areas, they may cause stress concentrations in those areas and promote further breakup of the roof beam.

\subsubsection{Mechanically Anchored (Expansion Shell) Bolts}

Historically, the majority of ground control efforts were accomplished using mechanically anchored bolts, particularly by pattern bolting of the roof. The current and projected use of mechanically anchored rockbolts in the underground is now limited in scope. The majority of currently planned tasks calls for the use of resinanchored threaded-bar bolts. One of the tasks still performed by mechanical anchors is the bolting of the ribs and roof for the purpose of hanging mesh to control spalling. Another use of the mechanical bolts is spot bolting of an area where limited size and condition warrants a small-scale bolting effort. Ground conditions and operational considerations may still require the limited local use of mechanically anchored bolts in the foreseeable future.

\subsubsection{Deformed Threaded Rebar}

Threaded-bar bolts with a limited-length resin anchor (e.g., 3 feet) are used for area pattern bolting as compared to limited spot bolting. For large areas needing roof support, pattern bolting with No. 7 (7/8-inch diameter) or No. 8 (1-inch diameter) threaded-bar bolts is the primary support system. Resin-anchored threaded-bar bolts provide load-bearing capacity and ductility superior to the mechanically anchored bolts historically used in Panel 1 and elsewhere at WIPP.

The threaded bars are anchored above the first clay seam to provide direct support of the roof beam, and are commonly used in conjunction with welded-wire mesh to contain smaller pieces of rock that may detach from the roof. To provide support for brows, a combination of resin-anchored threaded bar, cable shoes, and cable are typically installed. 
DOE/WIPP 97-2222

\subsubsection{Yielding Systems}

A variety of yielding systems are in place in the underground as small-scale and fullscale test emplacements. Threaded-bar bolts and cable bolts are being used with peripheral yielding components designed to yield with the creep-induced movement of the rock while at the same time providing dead-weight load support in the advent of rock detachment. The promise of these systems is that they will have a longer effective life by yielding by design to the creep process. Cable bolts also have an application in areas where roof height makes installation of a long rigid rockbolt difficult.

\subsubsection{Cable Mesh or Lacing}

The threaded-bar bolts may also be incorporated into a supplemental system of cable lacing. This type of support generally involves a grid system of wire-rope run transversely and longitudinally to form a square pattern. The wire ropes are woven under and over each other and supported at selected locations with the threaded-bar bolts. The intent of the cable lacing is to support small portions of detached rock that may not be controlled adequately by the existing threaded-bar bolts.

\subsubsection{Room 1, Panel 1, Support System}

This type of system provides a high level of confidence for ground control. A layered support structure of welded-wire mesh, expanded metal, channel steel, and pointanchored threaded bar provides intensive support. Load cells incorporated into each rockbolt provide load monitoring capability. Because of the cost of installation and long-term maintenance associated with this type of system, it does not lend itself to general ground control applications. In addition, this type of system does not address the problems associated with offset.

\subsubsection{Cribs}

Cribs have been used to a limited extent in areas currently closed to access. The primary function of the cribs has been to limit the extent of a roof fall that may occur in areas where continued ground control activities are no longer being performed (i.e., at the perimeter of closed areas).

\subsection{Removal of the Roof Beam}

The removal of the roof beam is not a support system but a mining alternative to ground support. The removal of the beam up to the next competent layer should be 
considered when adequate support cannot be provided in a cost-effective manner or if removal of the beam will result in a safer working environment. In existing drifts with anticipated long lives, creep closure may ultimately require additional excavation to maintain operational clearance. Field results of the beam removal in the East-140 Drift have shown this to be a viable alternative in areas of advanced beam deterioration. Observations, in the form of displacement measurements and fracture mapping, support the concept of removing the roof beam to enhance stability. Because many of the drifts that require long lives have already been mined, the effect of removing the roof beam well after initial mining has been investigated [U.S. Department of Energy, 1994].

\subsection{Area Closure}

The decision to close an area instead of supporting it involves administrative decisions as opposed to engineering solutions and, therefore, involves minimal labor and material costs. This option generally applies to areas that have a limited useful purpose, or if, from an economical and/or safety standpoint, it is more prudent to close the area than to maintain it. Closure of areas of the facility has been exercised in the past (e.g., all areas north of North-780). The option to close select areas of Panel 1 if safety becomes a concern is consistent with plans established in the 1994 Panel 1 Utilization Plan and the 1996 Long Term Ground Control Plan.

Consideration must also be given to the logistics of reopening an area if reopening is anticipated. The area of East-140 south of South-2180 will be reopened to service Panels 2 through 4 . This area has been closed for several years and its current condition is unknown. Significant ground control actions will probably be required prior to reentry of the area.

\subsection{REMEDIAL GROUND CONTROL ACTIONS IMPLEMENTED TO DATE}

All normally accessible areas of the underground except the South-90 electrical substation area are currently rockbolted. However, in some areas, such as the East-300 shop, the bolts do not penetrate the first clay seam and are only intended to support the mesh installed to control spalling. Early in the life of the facility, spot bolting was used to address areas of drummy or spalling ground that could not be scaled. Most of this spot bolting was done with 2-foot bolts. Beginning in 1989, the entire facility was pattern bolted with mechanical-anchor rockbolts. Bolts 10 feet in length predominate, although some areas have been bolted with 5-foot, 6-foot, or 8-foot bolts. 
Within the mechanical rockbolt systems, a 5-foot $x$ 5-foot offset pattern (5-foot $x$ 2.5-foot triangular) is the most common in the WIPP underground.

Because of limitations of mechanical-anchor rockbolts, more recent support system designs have focused on alternative types of rockbolts, primarily resin-anchored threaded bar. Other systems, such as cable bolts and yielding systems, are being tested and evaluated. In addition to using support systems to address ground control problems, excavation of the roof beam in a 600-foot section of the East-140 Drift was performed and the northern portion of the facility was closed to access eliminating the need for future ground control actions in the area. This section of the report presents an overview of ground control activities that have been initiated to supplement the mechanical-anchor rockbolt systems and to remediate areas of concern.

\subsection{Ground Support Systems}

\subsubsection{Panel 1}

Rockbolt support was installed in Panel 1 in 1988 using a rockbolt design based on the requirements for the demonstration program then in place. The original plan consisted of the storage of drums of contact-handled transuranic (CH TRU) waste in rooms for a period of 5 years. During this time and immediately following, the rooms were to be inaccessible, but the option to reenter was to be maintained so that the waste could be removed, if required. To assist with the possible reentry and to enhance stability, mechanically anchored rockbolts were installed. Ten-foot-long rockbolts were installed in Rooms 1 through 6, and 6-foot rockbolts were installed in Room 7, South-1600, and South-1950.

In 1991, a supplementary roof support system was designed and installed in Room 1 to facilitate a planned bin-scale test program. A detailed description of the supplementary system is presented in the Waste Isolation Pilot Plant Supplementary Roof Support System Underground Storage Area Room 1, Panel 1 [U.S. Department of Energy, 1991b]. Subsequently, additional ground support was installed, or installation is in progress, in all of the Panel 1 rooms and drifts with the exception to date of parts of Rooms 1 and 2. The roof-support history of Panel 1 is important because information on the age of the openings and when ground support was installed is vital to making predictions about future ground support requirements. A summary of additional support systems and year of installation in Panel 1 include: 
1991 - The support system referenced above was installed in Room 1.

1992 - A variation of the Room 1 system was installed in parts of Room 2.

1994 - Room 7 was rebolted with 13-foot-long No. 7 threaded bar with full-load nuts.

- The South-1600 Drift was rebolted with 13-foot-long No. 7 threaded bar with full-load nuts.

- The north half of Room 4 was rebolted with 13-foot-long No. 7 threaded bar with slip nuts.

- Room 5 was rebolted with 12-foot-long No. 7 threaded bar with full-load nuts.

1995 - The south half of Room 4 was rebolted with 12-foot-long No. 7 threaded bar with full-load nuts.

- Room 6 was rebolted with 12-foot-long No. 7 threaded bar with full-load nuts.

1996 - Room 3 was rebolted with 13-foot-long No. 7 threaded bar with full-load nuts.

- Rebolting of South-1950 with 13-foot-long No. 7 threaded bar with fullload nuts (in progress).

\subsubsection{East-140 Drift}

\section{North-460 to North-780}

Several arrays of yielding cable bolts are installed in this area. This is considered a full-scale emplacement/experiment. Nine arrays of bolts are (or were) installed with each successive array (north to south) having a designed yield 5,000 pounds higher then the previous array. The northernmost row of cable bolts have a designed yield of approximately 20,000 pounds, while the southernmost array has a designed yield of approximately 60,000 pounds. 
North-170 to North-460

A 5-foot $\times 4$-foot square pattern of rockbolts was installed in this area. The pattern consisted of 13-foot-long threaded-bar bolts with full-load nuts emplaced in $13 / 8$-inch boreholes.

\subsubsection{East-0 Drift - North-700 to North-780}

In this area, the existing system was reinforced with a 5-foot $x$ 5-foot triangular rockbolt pattern which was installed down the center of the drift only and which split the existing pattern. The pattern consisted of 13-foot-long threaded-bar bolts with full-load nuts emplaced in boreholes reamed to a diameter of 3 inches to approximately 1 foot beyond the first clay seam.

\subsubsection{East-0 and East-140 Drifts - North-780 to North-830}

Ground control measures were initiated as a component of the closing of the facility north of North-780. A 4-foot $\times 4.25$-foot square pattern of rockbolts was installed in both the East- 0 and East-140 Drifts from a point just south of North-780 extending to approximately North-830. The pattern consisted of threaded-bar bolts with full-load nuts emplaced in boreholes reamed to a diameter of 3 inches to approximately 1 foot beyond the first clay seam. The bolts emplaced were 12 feet long in the East- 0 Drift and 13 feet long in the East-140 Drift. In addition to the bolts, cribs were set just north of the barricades in each dritt.

\subsubsection{Brows}

Brows throughout the facility are provided with supplemental support because of visual evidence of separation. Wooden cribbing is used in areas where access is not a problem. Steel straps and wire-rope cable systems are installed on some brows at shaft stations and overcasts to provide additional support. A study is in progress to evaluate if there is a structural preference between a vertical brow and a brow cut at a 45-degree angle. Closure stations are installed on several brows to detect any acceleration in movement that may indicate a need for additional support.

\subsubsection{Control Of Broken Bolts}

In high-use areas and areas where bolt breaking is being observed, Mine Operations provides a secondary attachment of the rockbolts to the roof. A lanyard (safety wire, 
cable, or chain) is added to the system to prevent the rockbolt or any associated components from falling to the floor should the rockbolt fail. Geotechnical Engineering is assisting Mine Operations in evaluating different techniques to secure broken bolts.

The failure of rockbolts in the underground is a common experience, averaging one to two a day. Because of the safety factor calculated into the ground support design, a substantial number of bolts would have to fail before the integrity of the system would come into question. Therefore, the failure of a rockbolt does not pose a hazard from a ground control standpoint. However, there is a potential hazard associated with personnel being struck by a falling bolt. Over the years, several different methods of controlling the falling bolts have been employed, with varying degrees of success. Methods used have included wire mesh to hold the bolt in place and various lanyards made of wire, chain, or steel cable to catch a broken bolt. The wire mesh method made it difficult or impossible to identify when a bolt had failed. The lanyards appear to work well and make identification and removal of a broken bolt relatively easy. A method currently being tested is to loop a premanufactured stainless steel aircraft cable through the bearing plate or around the rockbolt and secure the cable to the roof with a powder-actuated nail and washer. Simulated field tests of this method have worked satisfactorily. Testing continues to evaluate the most cost-effective and efficient method to control rockbolt falls.

\subsection{Beam Removal}

\subsubsection{Salt Shaft Station}

It was proposed in the 1995 AGCOP that partial removal of the north brow at the Salt Shaft Station above the salt dump pocket be performed. Two conditions prompted this recommendation: fracture development in the brow and restricted truck dumping capacity (the bed of the truck when raised was contacting the roof), resulting from creep closure. The recommended work was completed. An area 20 feet in length, the width of the drift, and approximately 7 feet thick (up to the first clay seam) was removed.

The beam removal operation was begun using a Fletcher mechanical scaler. However, even though the rock was somewhat fractured, the rock proved to be very difficult to remove with the scaling machine. Partway through the operation, as the ground became less fractured, a Dosco roadheader machine replaced the scaler and removed 
DOE/WIPP 97-2222

the remaining portion of the beam. Threaded-bar bolts and cable straps are used to provide support to the newly created brow.

\subsubsection{East-140 Drift - South (Access to Disposal Panels)}

In 1994, the area of the East-140 Dritt from South-1300 to South-1950 was identified as requiring supplemental work. A system of threaded-bar bolts and wire-rope pairs was installed in select locations to provide additional support. Because of the size and age of the drift, deterioration continued, and a more permanent approach to addressing the problem was proposed. Removal of the roof beam to the first clay seam in the portion of the drift between South-1300 and South-1600 was prescribed. The proposed activities were approved, and removal of the roof beam for that section of the drift was completed in December of 1995 . The results of the beam removal were very positive. There were no roof falls ahead of the mining machine even though ground support was removed before excavation of the beam was initiated. The remaining upper beam is in excellent condition from a fracture standpoint and, unlike initial in situ mining, it has shown no discernible geomechanical response to the mining.

Based on these positive results, the scope of the beam removal operations in the East-140 Drift was expanded. The beam was removed from the South-1600 intersection to the north side of the South-1950 intersection, and from South-1000 to South-1300. Following beam removal in these areas, a preventative maintenance measure of installing mechanical-anchor rockbolts that do not penetrate the roof beam and chain link mesh at the rib roof intersection was employed.

\subsubsection{Lessons Learned}

The beam removal activities at the Salt Shaft Station and in the East-140 Drift have accomplished several goals that were anticipated prior to commencement of the work. These goals included development of removal techniques, mapping of roof-beam fracture patterns, observation and evaluation of in-place ground support as it was exposed, evaluation of the inherent stability of the fractured beam after support systems were removed, and creation of a competent roof.

\section{Removal Techniques}

Because of limited experience with removing roof beams at WIPP (the roof beam was removed at the Salt Shaft Station beginning in 1987), the initial removal activities in the East-140 Drift served as a training and development exercise. The roof beam that was 
previously removed at the Salt Shaft Station was relatively thin (about 2 feet thick) and fractured, which lent itself to removal with a mechanical scaler. The thicker beam (about 7 feet thick) on the north side of the Salt Shaft highlighted the difficulties associated with removing thicker beams with a scaling machine. It was seen that even though the beam was fractured, it was still quite competent. The Dosco roadheader mining machine was used to complete the beam removal at the shaft and for the entire beam removal process in the East-140 Drift.

Beam removal activities in the East-140 Drift were very successful and instructive. Initially, chain link mesh and other ground support components that were in place were removed ahead of the roadheader by using long-handled cutting torches that allowed for the torch operator to remain beneath the newly excavated roof. Although removing the ground support with a torch worked adequately, use of a mechanical scaler proved to be more efficient. The scaler, equipped with a spade bit, was used to peel away the support systems for 10 feet to 15 feet ahead of the mining machine.

\section{Fracture Mapping of the Roof Beam}

The beam removal process produced a vertical exposure or cross section of the roof beam. This exposure allowed for a written and photographed documentation of the fracturing systems observed in the beam. Approximately every 10 feet, the fractures were mapped showing their location, aperture, and orientation.

\section{Observation and Excavation of In-Place Ground Support}

The exposure of a vertical face of the roof beam also allowed direct observation of the ground support systems that had been in place for several years. Offset-induced bending in the mechanical-anchor rockbolts was documented and it was noted that the salt had crept or flowed around the bolt shafts.

Mining of the roof beam also provided the opportunity to evaluate the difficulty associated with mining through various types of in-place ground support. Overall it was determined that the types of support systems currently in use at WIPP do not pose a significant obstacle to the beam removal process. There was concern that cable bolts might wrap around the miner head, creating a problem. However, recent excavation of cable bolts indicates that they are probably easier to mine through than solid bars or bolts. 


\section{Evaluation of the Stability of the Fractured Beam}

A very positive aspect of the beam removal was the stability of the roof beam after the ground support was removed. Even with the ground support removed with the torch or scaling machine, there were no roof falls during the beam removal operation. This is significant in that, in what might be considered highly fractured areas, the beam retained enough strength to support itself even while the impactive process associated with the mining machine was under way.

\section{Creation of a Competent Roof}

The intent of removing the roof beam was to create a new roof that would require less maintenance and would provide a greater degree of safety. At this point, it appears that this goal was accomplished very satisfactorily.

\subsection{Area Closure}

All areas north of North-780 are now closed to access. Room $Q$ is also closed to access, as are the alcoves located in Panel 1. It is not anticipated that any further ground control activities will take place in these areas. The East-140 Drift south of South-2180 is closed at this time, but this area will eventually be opened and rehabilitated for waste emplacement operations in Panels 2 through 4.

\subsection{SUPPORT PROJECTIONS}

Based on the opening assessments and projections made in Section 2.0, several areas of concern are identified. They include areas that are operationally critical or exhibiting advanced deterioration as compared to other areas. Panel 1 is given special attention in these projections because of its unique requirements with respect to waste emplacement and the waste emplacement schedule. Table 2-2 lists time projections for support requirements. (A " 3 " indicates that work is projected to be necessary within 1 year; $a$ " 2 " indicates that work may be necessary within 5 years; and a " 1 " indicates that no work beyond normal maintenance is anticipated in the foreseeable future.)

In general terms, where reinforcing of existing support is required, a point-anchored threaded-bar bolt pattern with full-load anchor nuts will be used to supplement the existing pattern. In critical areas where cantilevering or fracturing of the beam is observed, a cable lacing system may be used, generally in conjunction with the 
threaded-bar system. If an opening is projected to be closed to access within a short time, mechanical bolts may be used to reinforce the existing support. The option to use cable bolts in areas where a thick roof beam and a low roof height make installation of long, rigid bolts difficult is aiso being considered. New excavations will probably be pattern bolted after the initial high creep response has passed (usually 1 to 3 years after excavation, depending on the opening geometry and nearby excavation). Specific plans and layouts will be prepared and will be based on a detailed evaluation of each area.

\subsection{Area Projections (Excluding Panel 1)}

Following are tentative support projections for specific locations in the underground. These projections are intended to provide planning guidelines for future work. Detailed designs will be prepared as necessary for construction. These support recommendations are based on a continued need to access these areas (i.e., unused rooms will not be abandoned or backfilled).

\subsubsection{All Areas North of North-780}

All areas north of North-780 are closed. No supplementary ground control actions are planned for these areas.

\subsubsection{East-0 - North of Salt Shaft Station Brow to North-780}

This area was excavated over 14 years ago and is now developing low-angle fracturing and some vertical fractures, although closure rates are relatively slow and steady. A pattern of threaded-bar bolts may be installed this fiscal year. Projected details follow:

Bolts: $\quad$ No. 7 threaded bar, 13 feet long, in 1-3/8-inch $\times 12.5$-foot holes reamed to 3 inches in diameter to approximately 1 foot beyond the first clay seam

Nuts: Full-load anchor nuts

Pattern: 4-foot $\times$ 4-foot nominal square pattern

Mesh: $\quad 4$-inch $\times 4$-inch $\times 1 / 4$-inch welded-wire mesh panels

Another bolting option under consideration for this area is the use of yielding cable bolts. The geometry of the openings (a thicker roof beam and a lower roof) make it difficult to install long rigid bolts. The flexibility of the cable bolts is more conducive to this situation. This area is a low traffic zone, and the installation of the yielding cable 
DOE/WIPP 97-2222

bolts would provide an opportunity for continued development and evaluation of the yielding systems.

\subsubsection{Parts of Air Intake Shaft North Access}

Small sections of the AIS North Access may require bolting in the coming year. These areas will be spot pattern bolted as needed. Bolting will be performed with threadedbar bolts or possibly with yielding cable bolts.

\subsubsection{Air Intake Shaft Station}

The inspection of this area indicates that the upper brow appears to be stable at this time. The lower brow is developing separation fractures. Because the rockbolts in place are only 6 feet in length and do not penetrate the first clay seam, it may be necessary to provide additional support in the station area in the form of a supplemental bolt pattern that does penetrate the clay seam. This will probably be necessary within the next 1 to 3 years. Details include:

Bolts: $\quad$ No. 7 threaded bar, 12 feet long, in 1-3/8-inch $\times 11.5$-foot holes reamed to 3 inches in diameter to approximately 1 foot beyond the first clay seam

Nuts: Full-load anchor nuts

Pattern: Split existing pattern

\subsubsection{East-300 - South-90 to North-250}

This area is not currently bolted except for short bolts that secure mesh. It will be necessary to pattern bolt this room within 1 to 3 years, with a possibility of it being done this year. Projected details follow:

Bolts: $\quad$ No. 7 threaded bar, 12 feet long, in 1-3/8-inch $\times 11.5$-foot holes reamed to 3 inches in diameter to approximately 1 foot beyond the first clay seam

Nuts: $\quad$ Full-load anchor nuts

Pattern: 4-foot $\times$ 4-foot nominal square pattern

Mesh: $\quad 2$-inch $\times 2$-inch $\times 1 / 8$-inch welded wire is already in place

Cable: None 
Another option under consideration for this area is the installation of yielding cable bolts. A decision on this option will be made following an evaluation of the in situ performance characteristics of the in-place and proposed yielding systems.

\subsubsection{East-140}

South-90 to North-250

Increased fracturing in this area may require some supplemental support in the next 5 years. Projected details follow:

Bolts: $\quad$ No. 7 threaded bar, 12 feet long, in 1-3/8-inch $\times 11.5$-foot holes reamed to 3 inches in diameter to approximately 1 foot beyond the first clay seam

Nuts: $\quad$ Full-load anchor nuts

Pattern: 4-foot $\times$ 4-foot nominal square pattern

Mesh: $\quad 4$-inch $\times 4$-inch $\times 1 / 4$-inch welded-wire mesh panels

North-250 to North-460

This area has recently been rebolted; however, continuing deterioration may require additional ground control actions in the next 5 years. Options for this area include cable lacing and removal of the roof beam.

\section{North-460 to North-780}

This area has recently been rebolted. Options for additional action in the next 5 years, if required, are identical to the North-250 to North-460 area with the exception that area closure may also be considered.

\subsubsection{East-140 - South (Access to Disposal Panels)}

Roof beam removal has been completed from South-1000 to South-1950 and the new roof is in excellent condition. The area of the drift from South-400 to South-700 is in good condition, and no remedial ground control activity is anticipated at this time. 
South-700 to South-1000

Increased fracturing in this area may require some supplemental support in the coming year. Projected details follow:

Bolts: $\quad$ No. 7 threaded bar, 12 feet long, in 1-3/8-inch $\times 11.5$-foot holes reamed to 3 inches in diameter to approximately 1 foot beyond the first clay seam

Nuts: $\quad$ Full-load anchor nuts

Pattern: 4-foot $\times$ 4-foot nominal square pattern

Mesh: $\quad 4$-inch $\times 4$-inch $\times 1 / 4$-inch welded-wire mesh panels

South-90 to South-400 and South-1950 to South-2050

Increased fracturing in these areas may require some supplemental support in the next 1 to 3 years. Projected details follow:

Bolts: No. 7 threaded bar, 12 feet long, in 1-3/8-inch $\times 11.5$-foot holes reamed to 3 inches in diameter to approximately 1 foot beyond the first clay seam

Nuts: Full-load anchor nuts

Pattern: 4-foot $\times$ 4-foot nominal square pattern

Mesh: $\quad 4$-inch $\times 4$-inch $\times 1 / 4$-inch welded-wire mesh panels

\subsubsection{Waste Shaft Station}

Because of salt creep and loss of pattern bolts in this area, it is anticipated that the support system in place will need to be augmented within 1 to 3 years. The success of the beam removal operation in the East-140 Drift makes beam removal an option for consideration at the Waste Shaft Station and other areas along the main haulage route to the waste disposal panels. A supplemental system consisting of pattern bolting with threaded-bar bolts may be employed. Details follow:

Bolts: $\quad$ No. 7 threaded bar, 10 feet long, in 1-inch $\times 11.5$-foot holes reamed to 3 inches in diameter to approximately 1 foot beyond the first clay seam

Nuts: Full-load anchor nuts

Pattern: Split existing pattern 
Mesh: Probably none beyond the chain link already in place

Cable: None

Scaling: Mechanical scaling of the area may be required before bolting is performed

\subsubsection{Salt Shaft Station}

The Salt Shaft Station had the roof beam removed approximately 8 years ago. The geotechnical monitoring in this area indicates that the roof has remained stable since that time. However, small vertical fractures have been observed and the current inplace rockbolts do not penetrate the existing roof beam. Because this is a high-use area, it may be prudent to install some additional bolts as a preventative maintenance measure within 5 years. The most likely supplemental support alternative would be to install No. 7 threaded-bar bolts through the existing chain link mesh.

\subsubsection{West-30 South-1300 to South-1600}

Because the pattern bolts in this area do not penetrate the beam, rebolting of this area may be, but is not anticipated to be, necessary within 5 years. Support, if required, will be provided by splitting the existing pattern. At the present time, continued observation is recommended.

\subsubsection{West-30 South-90 to South-400}

It is anticipated that some supplementary support will be necessary in this drift within 5 years. This will probably be installed as needed on a section-by-section basis. Projected details follow:

- Replace failed rockbolts with like bolts.

- Add a cable and welded-wire mesh system as required. The current bolt pattern density and bolt failure records indicate that the wire mesh can be attached with 2-foot-long mechanical-anchor rockbolts.

\subsubsection{West-30 South-1150 Booster Fan Area}

The angled brows created during the installation of the fans are a little different than what might be considered typical at WIPP. The brows are showing some fracturing, and some work will probably be required in the next 5 years. It is projected that the 
brow may be reshaped with a mechanical scaler as necessary and that some additional rockbolts will be installed.

\subsubsection{South-1000 Crosscut Between West-30 and West-170}

This is a high-use area and may require some supplemental support within 5 years. Projected details follow:

Bolts: $\quad$ No. 7 threaded bar, 13 feet long, in 1-3/8-inch $\times 12.5$-foot holes reamed to 3 inches in diameter to approximately 1 foot beyond the first clay seam

Nuts: Full-load anchor nuts

Pattern: 4-foot $\times$ 4-foot nominal square pattern

Mesh: $\quad 4$-inch $\times 4$-inch $\times 1 / 4$-inch welded-wire mesh panels

\subsubsection{South-1300 Crosscut Between West-30 and West-170}

This area may require some supplemental support in the next 5 years. Additional support may include splitting the existing support bolt pattern with No. 7 threaded-bar bolts. Mesh is currently in place on the roof.

\subsubsection{South-1600 Crosscut Between West-30 and West-170}

This area may require some supplemental support in the next 5 years. Projected details follow:

Bolts: $\quad$ No. 7 threaded bar, 12 feet long, in 1-3/8-inch $\times 11.5$-foot holes reamed to 3 inches in diameter to approximately 1 foot beyond the first clay seam

Nuts: Full-load anchor nuts

Pattern: 4-foot $\times$ 4-foot nominal square pattern

Mesh: $\quad 4$-inch $\times 4$-inch $\times 1 / 4$-inch welded-wire mesh panels

\subsection{Long-Term Low-Maintenance Areas}

In general, the remaining areas are in good condition, and it is anticipated that only routine maintenance, which includes scaling and spot bolting, will be necessary to maintain them over the next 5 years. 


\subsection{Panel 1 Detailed Projections and Options}

The schedule for the receipt of waste, both initial waste receipt and rate of receipt, is a crucial parameter in making ground control decisions relative to Panel 1. Because of the age of the panel and the continuing deformation taking place, the timing of waste emplacement will affect the support requirements relative to each room. Figure 6-1 presents the Baseline Repository Waste Emplacement Schedule.

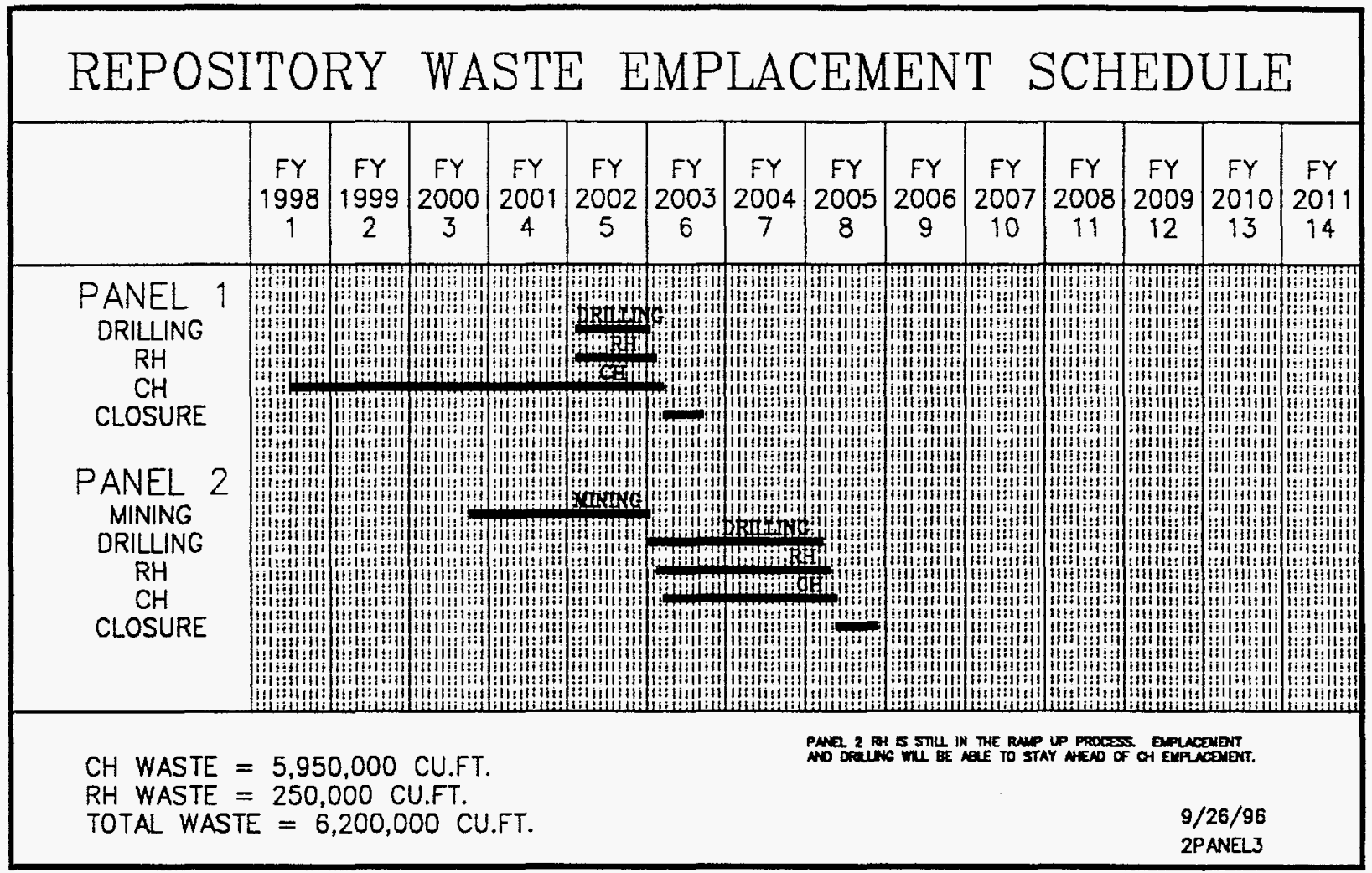

Figure 6-1. Baseline Repository Waste Emplacement Schedule

Panel 1 also provides an area for installation of new ground support systems. These systems have been installed to provide experimental performance data and to provide supplemental support. To the extent that it is practical within the space available, this area may continue to serve as a test zone for new ground control systems.

Support projections for specific areas of the panel are being planned based on the schedule presented in Figure 6-1. Deviations from the schedule can affect ground control preparation and other predisposal activities in two basic ways: (1) if the waste receipt schedule is accelerated, there may not be adequate time to install ground support systems as planned and (2) if the schedule is delayed, the ground control 
methods planned may not be adequate to sustain the ground without additional measures relative to the new time frame.

\subsubsection{Projected Conditions}

\subsubsection{Ground Conditions}

\section{Straight-Line Extrapolation}

Given the experience gained in the SPDV area, it was relatively easy to predict expected ground conditions for the first 10 years of Panel 1 life. However, as we move beyond that time frame, it becomes increasingly challenging to predict future conditions accurately. Some generalized statements relating to future ground conditions may be made:

- Fracturing will increase and become more notable, especially in the roof.

- Installed ground support may locally affect the roof fracturing processes.

- Room closure will continue and may affect operating clearances.

- Floor heave will become more apparent as the floor "detaches."

- Roof beam expansion rates may change because of fracturing and detachment processes.

If these processes proceed as a straight-line extrapolation, it is assumed that conditions will continue to deteriorate at the same rate as they have in the past, with no effect resulting from mining of Panel 2 or remote handled $(\mathrm{RH})$ waste boreholes. With normal maintenance and without any remediation, a rough estimate of a 1- to 5-year life is projected for all areas in the panel. The roof beam stability in individual rooms will increasingly be a function of the effectiveness of the installed roof support system(s). Regardless of the systems installed, it is apparent that the principal function will shift from helping the ground support itself to simply holding broken pieces of rock in place.

At this time, based on a straight-line extrapolation, it is believed that the addition of supplemental support systems or remediation by removing the roof beam can extend the life of all rooms in the panel through the Year 2002. However, the change in stress fields resulting from the mining of Panel 2 or the drilling of $\mathrm{RH}$ holes in Panel 1 pillars may have an adverse effect on Panel 1 room life. 


\section{Panel 2 Excavation Effects}

It is common knowledge in the mining industry that the distribution of rock stresses around an opening may be altered by nearby excavation. In the case of a room and pillar design, stress may be concentrated in the abutments or pillars of the outer rooms. Given a progressive sequence of mining, such as is the case with the mining of adjacent panels, the stresses will be redistributed and will tend to concentrate on the outer panels. In the case of WIPP, as Panels 2, 3 , and 4 are mined in sequence, stresses are expected to concentrate on the abutment north of Panel 1 and may accelerate closure processes in the panel. This is of no consequence or may be considered beneficial after panel closure. However, the redistribution of stresses from Panel 2 mining during Panel 1 waste emplacement may adversely affect Panel 1 room life because of its advanced age.

\section{Remote Handled Excavation Effects}

The mining of $\mathrm{RH}$ holes in pillars has been modeled and the effect has been shown to be that an increase in closure rates would occur. Based on the modeling and the planned $\mathrm{RH}$ hole excavation sequence, it is estimated that there will be an increase in the Panel 1 closure rates equivalent to about a year's normal closure (assuming no RH holes). Typically, this would be about three additional inches occurring as a result of the RH hole excavation. Although this sounds like an adverse effect, the "softening" effect on the pillars may, in the long term, reduce the rate of expansion in the immediate roof beam. The overall effect on ground conditions would likely be minimal.

\subsubsection{Support System Life}

All ground control systems used in the underground, including the yielding systems, have a finite life. Rockbolt failures are occurring throughout Panel 1. The failure of rockbolts is directly related to the length of time a bolt has been in place and the rock deformation rates in the area of the bolt. Knowing the mechanical properties of the support system components and the deformation rates of the rock permits an estimate of the effective life of the system to be made. The effects of axial deformation on a rockbolt and its expected life are fairly straightforward. If a bolt can withstand 6 inches of deformation before failure, and the rock deformation rate where the bolt is installed is 0.5 inch per year, that would translate into approximately 12 years of life for the bolt. What is not as straightforward is the effect of lateral loading on the bolt caused by stratigraphic offset. This lateral loading appears to be at least partially responsible for 
the majority of current bolt failures. This failure mechanism makes predictions of support system life very difficult. However, oversizing of the rockbolt boreholes and terminating the resin column 1 foot above the clay seam should aid in extending the working life of the threaded-bar bolts.

As the age of the rockbolt systems increases, the number of bolt failures will increase. Current procedures call for replacement of failed rockbolts as required. Lanyard-type safety devices such as chains, wires, and cables are being attached to select bolts to prevent them from becoming a safety hazard in the event of failure. As the rate of bolt failures increases, the replacement of the bolts could become a significant maintenance effort.

\subsubsection{Detailed Plans And Options}

This section provides options for panel usage and the ground control alternatives required to implement those options. Evaluation of specific areas and recommendations for remedial ground control procedures must be made in a time frame that allows for implementation of those procedures prior to waste emplacement. Under all options, general maintenance activities, such as scaling down small pieces of rock and replacing identified broken bolts as required, would continue in all accessible areas. The identified options are:

- Use all of Panel 1.

- Receive first waste in Panel 1 and use as much of the panel as possible.

- Receive first waste in Panel 1 and move to Panel 2 as soon as possible.

- Close Panel 1 and begin initial waste emplacement in Panel 2.

In the case of several of the options and alternatives, significant overlap exists. For example, the current plan, consistent with the 1994 Panel 1 Utilization Plan, is to receive waste in Panel 1 and use as much of the area as possible. If waste is received in 1998 and emplacement proceeds expeditiously, it is probable that all of the panel will be used to emplace waste. However, if delays are experienced in waste receipt or in the emplacement sequence, portions of the panel may not be used or substantial efforts may be required to maintain the ground.

\subsubsection{Use All of Panel 1}

The waste receipt schedule as discussed in Section 6.3 is a critical parameter when planning to use all of the panel. The Baseline Repository Waste Emplacement 
Schedule as presented in Figure 6-1 will be used for the following alternative discussions. Based on this schedule, it is reasonable to expect at this time that with the use of proven ground control techniques, all of Panel 1 can be used for waste disposal. However, if there is a significant delay in the baseline schedule, the preference of alternatives may change. For example, a 5-year delay in the schedule may make beam removal options more attractive.

\section{Alternative 1 - Install supplemental support of cable lacing in conjunction with rebolting and mining down of fractured roof beams as required.}

Under this alternative, broken bolts in active support systems will be replaced as they are identified. Current plans call for installation of supplemental ground support in each room prior to waste emplacement. Because ground control activities will not be performed in areas where waste has been emplaced, it is desirable to install the supplemental systems as near to the time of waste emplacement as possible. Given the planned waste receipt rates, this will ensure that the system will remain effective through the waste emplacement period for the area. A preferred system at this time would consist of a combination of threaded-bar bolts for primary support, mechanicalanchor bolts for supporting smaller pieces of rock near the ribs as required, weldedwire mesh throughout to contain small rock, and a square pattern of 5/8-inch steelcable lacing to support larger pieces of rock that may hang or detach.

Sections of rock may be scaled down or, in more extreme cases, portions of the roof beam may be excavated or mined before the supplemental system is installed. Mining of the roof beam is the most time-consuming component of this alternative. Adequate time must be allowed to accomplish this task, if required. If the entire beam is removed throughout the room, the mining sequence will take approximately 6 months, based on East-140 roof-beam mining rates. If beam removal is employed, backfilling of the floor will be required to maintain the as-designed and permitted size of the disposal rooms. Dependent on the degree of beam removal planned, several months may be required for each section of beam to be removed. For example, if a large section of the beam was to be removed in Room 7, the first room scheduled to receive waste, those activities would need to be initiated immediately to meet the mid-1998 waste receipt schedule. On the other hand, if the only room anticipated for beam removal activities was Room 1, those actions could be initiated sometime after initial receipt of waste.

Alternative 2 - Install supplemental support of cable lacing in conjunction with rebolting and installation of external support as required. 
The rebolting and cable lacing system will be the same or similar for this alternative as that for Alternative 1. Installation of external support, such as cribs, could be performed before or after the internal support was installed. The external support will be placed in areas of concern, such as along a rib where the roof had extensive lowangle fracturing or in the center of a room where closure rates may be greater. External support will decrease the disposal capacity of the room, and consideration must be given to its possible interference with waste handling operations.

Alternative 3 - Mine the roof in the entire panel.

To exercise this option under the baseline waste receipt schedule presented would require that beam removal activities begin in the near future. It would be desirable to have two or three rooms completed prior to initial waste receipt in order to be assured of staying ahead of the waste disposal operations.

The most likely scenario for this alternative to be considered viable would be in the case of a significant delay in the initial receipt of waste. This is mainly because of the time required to implement this alternative. However, if the timing was such as to allow for the beam removal to take place, preliminary results of the beam removal in East-140 indicate that this process would extend the useful life of the panel.

6.3.2.2 Receive the First Waste in Panel 1 and Use as Much of the Panel as Possible Alternative 1 - Supplemental support of cable lacing in conjunction with rebolting and closing individual rooms only as required.

This option is the current course being followed and is consistent with plans established in the 1994 Panel 1 Utilization Plan. This plan does not exclude the alternative to use all of the panel, but includes the option to close a room or area if determined prudent.

Following this plan, broken bolts will be replaced as they are identified. Supplemental support will be installed in the area chosen for initial emplacement as near to the time of waste receipt as possible. As disposal operations progress, the viability of using rooms will be assessed primarily from a safety standpoint, but also with economic and operational considerations in mind. If it is determined prudent, a room or rooms could be closed. Factors other than the stability of the rooms that may affect this decision 
include the availability of Panel 2, the anticipated volume of waste to be received, and the rate of waste receipt and emplacement.

Using the baseline waste receipt schedule as a guide and taking into account the current age and condition of the Panel 1 rooms, a rough prediction of which rooms may be at risk can be made. Rooms 1, 2, and 3 are 1 to 2 years older than Rooms 4 through 7. Additionally, the sequence of waste emplacement begins with Room 7 and progresses to Room 1 over a period of approximately 4.5 years. This means that when waste is emplaced in Room 1, it will be approximately 6 years older than Room 7 was when waste was emplaced there. Logic dictates that the rock deformation associated with this 6-year period will increase the probability of Room 1 experiencing unacceptable ground conditions. Hence, Room 1 would be the most likely room to be closed and abandoned. Because the rooms were excavated generally in the opposite order of which they will be used, all things being equal, it seems reasonable to assume that Room 2 would be the next logical choice for abandonment and so on down to Room 7. However, all things are not necessarily equal. At the present time, Room 3 is in better condition than Room 4. This means, for example, it is possible that Room 4 could be abandoned and Room 3 used.

\subsubsection{Receive the First Waste in Panel 1 and Move to Panel 2 as Soon as Possible}

Alternative 1 - Supplemental support of cable lacing in conjunction with rebolting and move to Panel 2 as soon as it is ready.

Installation procedures for supplemental ground support under this option are identical to previous alternatives. As much of Panel 1 would be used as required until Panel 2 was ready for disposal operations. Several variables come into play under this alternative that will affect which rooms of the panel may or may not be used. The key variable will be the anticipated completion time for Panel 2. Assuming the completion date for Panel 2 and the rate of waste receipt is known, a fairly accurate estimate can be made of the disposal space required in Panel 1, allowing the luxury of selecting the best rooms for use. If, for example, Room 7 was nearly filled with waste and Panel 2 was scheduled for completion in 4 months, and knowing that 4 months' worth of waste would not fill an entire room, any of the remaining rooms could be selected. The room used would probably be the one determined to be in the best condition. 


\subsubsection{Close All of Panel 1 and Begin Initial Waste Emplacement in Panel 2}

No further ground control activities in Panel 1 would be required under this option. Pertinent factors in this decision would be the consequences from a regulatory, operations, and performance assessment standpoint of closing Panel 1. Some of these consequences are discussed in the 1994 Panel 1 Utilization Plan.

\subsection{SUMMARY STATEMENT}

This document is used for planning purposes only. Ground conditions at WIPP are dynamic and, as such, projections for ground control actions as presented in this plan may change. The schedule for receipt of waste also affects the projections as presented. Significant delays in the receipt schedule would most likely result in significant changes to current ground control plans. Geotechnical Engineering evaluates a wide variety of current geomechanical and observational data to formulate these projections.

\subsection{REFERENCES}

Holt, R. M. and D. W. Powers, 1984. Geotechnical Activities In The Waste Handling Shaft, Waste Isolation Pilot Plant (WIPP) Project, Southeastern New Mexico, Westinghouse Electric Corporation, Waste Isolation Pilot Plant, Carisbad, New Mexico.

Garcia, J. J., 1996. Transmittal of the 1996 Update to the 1994 Panel 1 Utilization Plan, WD:96:02147, Attachment: Panel 1 Utilization Plan 1996 Update, Letter to E. K. Hunter, Department of Energy, Carlsbad Area Office, Carlsbad, NM, October 31.

U.S. Department of Energy, 1991a. Report of the Geotechnical Panel on the Effective Life of Rooms in Panel 1, DOEMIPP 91-023, Waste Isolation Pilot Plant, Carlsbad, New Mexico.

U.S. Department of Energy, 1991b. Waste Isolation Pilot Plant Supplementary Roof Support System Underground Storage Area Room 1, Panel 1, DOE/WIPP 91-057, Waste Isolation Pilot Plant, Carlsbad, New Mexico.

U.S. Department of Energy, 1993a. Geotechnical Field Data and Analysis Report, DOE/WIPP 93-019, Waste Isolation Pilot Plant, Carlsbad, New Mexico. 
U.S. Department of Energy, 1993b. The Current Bases for Roof Fall Prediction at WIPP and a Preliminary Prediction for SPDV Room 2, DOE/WIPP 93-033, Waste Isolation Pilot Plant, Carlsbad, New Mexico.

U.S. Department of Energy, 1994. Investigation of the Advantages of Removing Highly Fractured Roof Beams, DOEMIPP 94-025C, Waste Isolation Pilot Plant, Carlsbad, New Mexico.

U.S. Department of Energy, 1996. Geotechnical Analysis Report for July 1994 June 1995, DOEMIPP-96-2193, Waste Isolation Pilot Plant, Carlsbad, New Mexico.

Westinghouse Electric Corporation, 1994. 1994 Panel 1 Utilization Plan, WIPPMID-94-2027, Waste Isolation Division, Carlsbad, New Mexico. 
APPENDIX A

\section{SUPPORT TECHNOLOGIES}




\section{APPENDIX A \\ SUPPORT TECHNOLOGIES}

\subsection{EVALUATION AND IMPLEMENTATION}

The Ground Control Section of Geotechnical Engineering continues to investigate new or alternative technologies for use in underground support. Supplemental systems consisting of full-load threaded-bar bolts are in place in many areas. These systems are relatively young, and their monitoring and evaluation will continue. A large amount of testing has concentrated on yielding systems. Yielding systems that can yield along with normal rock creep and deformation, but still provide substantial support as roof strata break up or detach, may offer the greatest advantages for long-term ground support. It is anticipated that they should perform better than rigid systems with regard to creep and offset loading, but still provide the required dead-weight load support. The threaded-bar bolt systems currently in use in the underground are performing well from the standpoint of providing excellent load-bearing capacity. However, they are not expected to perform as well in offset. Cable bolts with yielding anchors or yielding collars may address these criteria. Laboratory testing to evaluate these technologies was performed and the results are promising. Additional in situ testing of yielding cable systems is in progress.

New technologies will be evaluated as they become available and will be used as they are proven. Given the requirement to maintain underground openings for up to 50 years, and with few alternatives for relocation of haulageways, it is expected that underground support systems will evolve and become more refined and complex through the life of WIPP.

\subsection{Ground Control System Testing}

Testing of various ground support components and systems is currently being performed. This testing program will be expanded as necessary to more fully evaluate and document the operational performance of proposed and in-place systems. In situ testing also serves as a means of developing and/or perfecting installation procedures for new systems. 


\subsubsection{No. 7 Grade 60 Threaded Bar With Dywidag Slipnut}

Dywidag Systems Inc. has developed a nut for use on the threaded bar that is capable of cutting threads from the bolt within a variable load range (i.e., the nut slips down the threaded bar). The load at which the nut slips is determined by the design of the nut, but would be somewhat lower than the yield strength of the bolt. The intent of the slipnut is to allow the system to yield with the creep closure of the opening. The system allows for the addition of a full-load nut if the slipnuts do not perform as desired.

Results of laboratory direct-pull tests indicate that the slipnut will yield well below the yield point of the bolt as the nut cuts threads and moves down the bolt shaft. When the slipnut yields, the load on the bolt drops to zero or near zero, which results in cyclic loading during the displacement phase. Results of offset loading tests indicate that systems that are allowed to yield at the collar end are less likely to fail at the point of offset than systems that are fixed at the collar end.

\section{Room 4 System}

A full-scale emplacement of the threaded bar and slipnut system was installed in the north half of Room 4, Panel 1. This system also incorporates rockbolts equipped with strain gages and reflective tape attached to each bolt to aid in monitoring the slip of the nuts. When a nut slips, it covers the reflective tape, making it easy to see when a slip has occurred. After each slip, a different colored tape is attached to the bolt to indicate how many slips have taken place (white $=$ one slip, red = two slips, green $=$ three slips, etc.). The ability to monitor the rate and amount of yield on the bolts in this support system has provided the additional benefit of seeing how load values and rates vary throughout the emplacement. Several areas within the emplacement have had very little movement, indicated by no slipnut movement, while other areas have had as much as 4 inches of movement indicated by eight slips of the slipnuts. The system is performing largely as expected; therefore, the current course of monitoring will be continued. This type of monitoring will stop when the yielding-tail length is used up.

\subsubsection{No. 7 Grade 60 Threaded Bar With Yielding Rockbolt Insert}

This system allows for a yielding component to be inserted between the full-load nut and the bearing plate. The type of yielding insert can vary, and various types of inserts have been tested in the laboratory and in situ to determine their performance characteristics. Titan Load Indicators are now in use in several areas of the facility, and they are performing according to design. 
The Titan Load Indicator is a metal cylinder with machined grooves that enable the cylinder to collapse at selected loads. The precision design of the insert allows it to serve as a load indicator, while the collapsible feature allows it to serve as a yielding component. Direct-pull tests were performed with the load indicator installed on a rockbolt. The indicators tested each had three grooves, which all yieided at different loads as prescribed. During yielding there was only a small reduction in bearing capacity followed by load buildup to the next yield point. These load indicators are installed on several support systems at WIPP and are working well. When installed on all or a majority of bolts in a support pattern, they provide a means to evaluate the distribution of loads throughout that area similar to the slipnuts in the Room 4 system.

\section{Room 2 Test}

Titan Load Indicators rated at 20,000 pounds were recently added to a threaded-bar support system in Room 2. This system is being detensioned when the loads reach approximately 20,000 pounds as indicated by load cells. The addition of the load indicators appears to have extended the time required between detensionings from about a month to about 1 year.

\subsubsection{Tensionable Cable Bolt With Dywidag Slipnut}

Dywidag manufactures a tensionable cable bolt consisting of a 0.6 -inch-diameter, grade 270 cable, which terminates on the collar end with a No. 7 grade 60 threaded bar. The threaded bar is compatible with their slipnut that results in a yielding system.

Direct-pull tests of the tensionable cable bolts with slipnuts installed were performed. The nuts slipped with uniform peaks, with the load dropping to zero, and then reloading to the next slip. A noted difference between these tests and previous tests with Dywidag bar is that the tails tended to form a dogleg beyond the slipnut during load application. The tails exhibited or retained notable curvature after testing was completed. Four of these tensionable cable bolts are currently in place and being monitored at WIPP.

Offset load testing was also performed on this system. The yielding nut allowed the cable to be laterally offset several inches without failing. 


\subsubsection{Yielding Cable Bolt}

This bolt is currently in a prototype stage, but initial laboratory testing has been performed with good results. The system consists of a 0.6 -inch-diameter, grade 270 cable with a yielding component on the collar end. The yielding component consists of a bullet-shaped tube installed on the center strand of the cable that increases its effective diameter. The section of the cable containing the tube is pulled through a confining cylinder or slide chamber. The tube installed on the center strand can be varied in diameter and length to produce various yield characteristics. The allowable displacement is limited only by the length of the slide chamber, which is adjustable.

Direct-pull tests of this system were performed. In all cases, a straight elastic loading was followed by the yielding of the cable through the slide chamber. In general, peak load was reached after about 8 inches. Yielding was very constant at approximately \pm 5 percent of the peak load. No component failures were noted. The test series was varied to gather data for yielding systems with peak loads varying from approximately 50,000 pounds to under 20,000 pounds.

\section{East-140 Drift Emplacements}

Full-scale in situ tests of this system are currently in place in the form of a supplemental support system in the East-140 Drift between North-460 and North-780. Designed yield ranges of the in-place systems range from 20,000 pounds to 60,000 pounds. Preliminary results of the in situ emplacement indicate that the bolts are yielding at levels somewhat lower than in the laboratory (i.e., lower than their design yield). This yield difference may be attributed to installation techniques and/or the short time span associated with a laboratory test in contrast to the longer time frames associated with an in situ installation.

\section{Testing in Other Mines}

In addition to the systems installed at WIPP, cooperative work with other mines is ongoing. Emplacements of yielding cable bolts in commercial mines with similar yielding rock types (potash and trona) are in place at this time. To date, none of the bolts installed in the potash mine have reached yield. While overall ground movement has been significant, differential movement between the anchor point and the collar of the bolts is limited. A few bolts in the trona mine installation have reached yield. However, no instrumented bolts have yielded at this time. Monitoring of these systems 
continues and should provide information on how these support systems will respond to a variety of loading scenarios.

\subsection{New Systems}

A rockbolt under consideration for use at WIPP is the German made Kombibolt. As of this writing a quantity of these bolts are on order for test purposes. This bolt is a combination point-anchored and fully grouted bolt with yielding components. An outer sleeve is fully grouted into the formation, while an inner bolt shaft is point anchored at depth and is allowed to move freely within the outer sleeve. Yielding components are integrated into the collar end of the system.

\subsection{ADVANTAGES AND DISADVANTAGES OF GROUND CONTROL SYSTEMS}

\subsection{Mechanically Anchored (Expansion Shell) Bolts}

The advantages of the mechanical rockbolt system being used at WIPP include:

- They are simple to install.

- They are relatively inexpensive and readily available.

- Because of their wide use in the local potash basin and at the WIPP site, their support capabilities and limitations are known.

- They install flush with the rock surface and, therefore, do not impose significantly on drift dimensions, which can be an operational concern.

- The point-anchor system allows for the strain in the bolt to be distributed over the entire length of the bolt.

The disadvantages of the mechanical rockbolt system being used at WIPP include:

- The high-grade steel used in the installed bolts allows a limited amount of elongation or flexure, as compared to a yielding system, before failure occurs. This characteristic makes those bolts less desirable for long-term use because of the creep closure and offset movement associated with the openings at WIPP. Additionally, a materials evaluation of the mechanical-anchor-type rockbolts used at WIPP revealed that there was considerable variation in content; for example, carbon content, from bolt to bolt. The variability in content means that each bolt may respond differently, to some degree, to similar loading conditions. 
- Improper installation can cause uneven loading of the bolt head and premature head failure. The bolts are also susceptible to overtorquing during installation, which can result in premature failure.

- The anchorage capacity of the mechanical bolt may be less than the yield strength of the bolt.

- The possibility of corrosion effects or of embedment of the leaves of the anchor into the formation may result in premature shell anchor slippage or failure. However, the number of recorded anchor failures is minimal.

\subsection{Deformed Threaded Rebar}

The advantages of the resin-anchored threaded bar system include:

- The anchorage pullout strength exceeds the bar strength when the bolt is properly installed.

- The design allows for pretensioning or detensioning of the bolt after emplacement.

- The threaded bar can be cut on site to any length desired.

- The No. 7 threaded bar at $7 / 8$ inch in diameter is slightly larger than the $3 / 4$-inch mechanical-anchor bolt. The threaded bar is made of grade 60 steel, which is more ductile than the grade 75 steel of the mechanical-anchor bolt. The more ductile threaded bar should be more flexible in response to the creep closure and stratigraphic offset observed at WIPP (preliminary laboratory testing indicates this to be the case). Although the threaded bar is a lower grade steel, the larger diameter of the 7/8-inch threaded bar results in an ultimate stress limit approximately 62 percent greater than the 3/4-inch mechanical-anchor bolt. This is based on an ultimate strength for 3/4-inch mechanical-anchor bolts of 33,400 pounds, as listed in the American Society of Testing and Materials (ASTM) F432, and an ultimate strength for $7 / 8$-inch threaded bar of 54,000 pounds, as presented in manufacturers' specifications.

- The system is readily adaptable for use with yielding components such as slipnuts, load indicators, or yielding inserts. 
- The point-anchor method of securing the bolt, as opposed to fully grouting the bolt, allows the strain to be distributed over the entire free length of the bolt.

- The anchor zone and length can be varied.

The disadvantages of the resin-anchored threaded-bar system include:

- When using the system as a yielding system with slipnuts or yielding inserts, a tail must be left extending from the borehole. This protruding tail is generally 12 to 18 inches long. This can be an operational concern based on the roof height of the area to be bolted.

- The installation of the threaded bar is more complicated than that of mechanically anchored rockbolts because of the use of a resin-anchoring system. Care must be taken in the handling and storage of the resin cartridges. It is also important to allow for proper mixing and setting of the resin anchor.

- A materials evaluation of the threaded-bar rockbolts used at WIPP revealed that there was considerable variation in content; for example, carbon content, from bolt to bolt. The variability in content means that each bolt may respond differently, to some degree, to similar loading conditions.

\subsection{Cable Bolts}

The advantages that are anticipated through the use of a cable bolt system include:

- Preliminary laboratory testing indicates that cable bolt systems may react more favorably to offset loading observed at the site than the more rigid bolting systems.

- The cable bolt allows yielding components to be attached to the collar end, providing a system that has favorable attributes from both an axial and lateral loading perspective.

The disadvantages related to the use of the cable systems currently include:

- The cable bolt is more difficult to install than the previously mentioned systems. The installation requires an experienced crew to ensure that the resin cartridges are completely mixed.

- Corrosion may have a greater detrimental effect on the cable system because of the larger exposed surface area associated with the design. Methods of coating the cables to isolate them from the corrosive environment have been developed by 
manufacturers. However, the efficacy of the coatings and the effect of coatings on installation and anchor performance need to be evaluated.

\subsection{Cable Mesh}

The advantages to using a supplemental cable mesh system include:

- A cable mesh system provides a lightweight support system that supplements an existing or proposed system and requires a minimum amount of rockbolts for installation.

- The cable mesh system can be installed easily over an existing support system. The system allows for the support of a roof beam that is experiencing advanced fracturing.

The disadvantage inherent to the cable mesh system includes:

- High loads can be developed in the cable when it is loaded perpendicular to its length. Because of the unusual manner in which salt creep and associated lateral deformations may load the system, definitive load/design criteria have not been established for these systems.

\subsection{Room 1, Panel 1, Support System}

The advantages of the Room 1, Panel 1, support system include:

- This system provides a high level of support system monitoring.

- The design and installation of this system was inspected and reviewed and has received numerous approvals from state and federal regulatory agencies.

- The system, as installed, is monitored for load buildup. Bolts are detensioned periodically to prevent them from reaching their yield strength. The adjustable nature of the system allows for long-term yielding support.

- The system has load cells on every threaded-bar bolt to allow for constant monitoring of the load. This monitoring should provide an indication of any failure of a bolt within the system. 
The disadvantages of the Room 1, Panel 1, support system include:

- The installation of the system is very labor intensive, and the cost of the system is comparatively high. These factors make this type of system economically impractical to apply throughout the mine.

- The system requires continued monitoring and adjustment to meet the design criteria. The constant maintenance associated with this system also makes it less cost effective.

- Detensioning of the rockbolts at relatively low confining loads (e.g., detensioning of the bolts at 20,000 pounds) may not restrict the fracture propagation or slow beam expansion rates as rigid bolts appear to do.

- The adjustable aspect of the system is accommodated with longer threaded-bar bolts that initially protrude into the drift approximately 18 inches.

\subsection{Removal of the Roof Beam}

The advantages of roof-beam removal presented in the report are:

- The geotechnical field data indicate that roof fracturing and displacement are minimized in an excavation with the roofline located at or immediately above a clay seam.

- The field data indicate that removal of a highly fractured roof beam significantly improves the stability of the subsequent excavation even though it is larger.

- Numerical analyses indicate that drifts with the roofline located at or immediately above a clay seam develop less strain in the roof.

- Numerical analyses indicate that the removal of a highly deformed roof beam significantly improves the condition of the root.

- The life of drifts with highly fractured roof beams can be significantly extended by removing the roof beam to the nearest clay seam.

- Field observations in the East-140 Drift where the beam was removed support the advantages listed above.

The disadvantages associated with the root-beam removal include:

- Beam removal is more labor intensive in the short term than traditional methods of ground support. 
- The new rootmay me more difficult to observe and maintain because of the increased height.

- The new roof beam may be thinner at the centerline of the opening because of the excavation technique. Excavation of the beam is performed by mining up to clay $G$ near one rib, then swinging the miner arm horizontally across to the other rib. Because the cut is horizontal, a sagging upper beam will have more material removed at the opening centerline.

- The increased opening dimension increases potential subsidence magnitude (if applied extensively).

- The alteration of drift dimensions may require an adjustment of the ventilation specifications for a particular area.

- Numerical modeling indicates increased convergence rates.

\subsection{Closure of Selected Areas}

The advantages of area closures include:

- Continuing maintenance of the area would be eliminated.

- Safety concerns, if any, associated with the area to be closed would be eliminated.

The disadvantages of area closures include:

- This may affect material storage and/or in situ testing operations. The area could no longer be used.

- If it is anticipated that the area may be reopened in the future, the costs associated with rehabilitation of an area that has not been maintained may be more than the cost of continued maintenance. 